\title{
INTERSECTIONS ON TROPICAL MODULI SPACES
}

\author{
JOHANNES RAU
}

\begin{abstract}
This article explores to which extent the algebro-geometric theory of rational descendant GromovWitten invariants can be carried over to the tropical world. Despite the fact that the tropical moduli-spaces we work with are non-compact, the answer is surprisingly positive. We discuss the string, divisor and dilaton equations, we prove a splitting lemma describing the intersection with a "boundary" divisor, and we prove general tropical versions of the WDVV, respectively, topological recursion equations (under some assumptions). As a direct application, we prove that, for the toric varieties $\mathbb{P}^{1}, \mathbb{P}^{2}, \mathbb{P}^{1} \times \mathbb{P}^{1}$ and with $\Psi$-conditions only in combination with point conditions, the tropical and classical descendant Gromov-Witten invariants coincide (which extends the result for $\mathbb{P}^{2}$ in [17]). Our approach uses tropical intersection theory and unifies and simplifies some parts of the existing tropical enumerative geometry (for rational curves).
\end{abstract}

Introduction. Over the last few years, the list of results in tropical enumerative geometry has become quite long. However, lacking an appropriate tropical intersection theory, most existing results are obtained by:

- relating the tropical numbers directly to the classical ones (cf., [18]) and then using the algebro-geometric theory, or

- involving ad hoc computations (eg., $[3,5,9,14,17]$ ), which moreover, have to be repeated for each new class of enumerative problem.

On the other hand, based on [19], the basic constructions of tropical intersection theory are now developed in $[\mathbf{1}]$ (see also $[\mathbf{2}, \mathbf{1 3}]$ ). Further-

2010 AMS Mathematics subject classification. Primary 14T05, Secondary $14 \mathrm{~N} 35,52 \mathrm{~B} 20$.

Keywords and phrases. Tropical Gromov-Witten theory, tropical intersection theory, tropical geometry.

Received by the editors on February 24, 2014. 
more, in [8], the authors show that the moduli spaces of rational tropical curves are tropical varieties (i.e., satisfy the balancing condition). Hence, we can apply intersection theory to them. In [20], Mikhalkin proposes the definition of tropical $\Psi$-divisors in tropical moduli spaces of abstract curves, and they were first studied in [15]. In summary, all the tools needed to develop a tropical analogue of classical GromovWitten theory for rational curves are at our disposal, and the present article tries to carry out this program (as mentioned before, first steps are contained e.g., in $[8,15,17])$.

The "ready for use" main Theorems 5.18 and 5.20 state that, for $\mathbb{P}^{1}$, $\mathbb{P}^{2}$ and $\mathbb{P}^{1} \times \mathbb{P}^{1}$, and with $\Psi$-conditions only in combination with point conditions, the tropical and conventional descendant Gromov-Witten invariants coincide. For the case of $\mathbb{P}^{2}$, this equality was already proven in the previous paper [17] joint with Markwig. One should emphasize that both results are obtained by checking that the involved numbers satisfy the same recursive formulas, and not by proving some sort of correspondence theorem.

This article is a continuation of [17], and some statements can be found in older versions there. The focus here is to consequently replace older ad hoc computations by more appropriate tools from tropical intersection theory. As a consequence, we typically obtain more general statements (e.g., working in any dimension).

We work with non-compact tropical moduli spaces, i.e., fans in $\mathbb{R}^{N}$, due to the fact that compactifications and their intersection theory have not yet been satisfactorily constructed. However, the noncompact approach has its limitations. This will become visible, e.g., from the assumptions we need in our general WDVV and topological recursion statements.

Let us also mention that in subsection 1.4 we show that the fan displacement rule for Minkowski weights describing toric intersection theory (cf., [7]) coincides with the intersection product of tropical cycles introduced in [1, Section 9] (see also [13]).

The article contains the following parts. Section 1 repeats the basics of tropical intersection theory from [1] and adds some results which will be important later. In Section 2, we study the intersection ring of $\mathcal{M}_{n}$, the space of abstract rational tropical curves. In Section 3, 
we extend this to $\mathcal{M}_{n}^{\text {lab }}\left(\mathbb{R}^{r}, \Delta\right)$, the space of parametrized curves in $\mathbb{R}^{r}$. In particular, we prove general versions of the string, dilaton and divisor equations. Section 4 deals with the intersection of a onedimensional family of curves with a boundary divisor. By analogy with the classical case, we prove a "splitting lemma" which allows computing this intersection as a product of intersections on smaller moduli spaces. Finally, in Section 5, we put things together and prove, under some conditions, the WDVV and topological recursion equations mentioned above.

1. Intersection theory. In this section, we will establish the parts of tropical intersection theory that we will need to attack the problems of tropical Gromov-Witten theory in a satisfactory way. Subsection 1.1 gives a quick overview on the definitions and results from $[\mathbf{1}, \mathbf{2}]$ that we will need (however, note that our notation will sometimes differ slightly from the original ones). Subsections $1.2-1.7$ contain some new material. In particular, subsection 1.4 contains a proof of the fact that toric intersection theory (as described by the fan displacement rule in [7]) and tropical intersection theory for fans are identical. (An alternative proof can be found in [13, Theorem 4.4]).

1.1. The basics. A cycle $X$ is a balanced (pure-dimensional, weighted, rational and polyhedral) complex in a finite-dimensional vector space $V=\Lambda \otimes \mathbb{R}$ with underlying lattice $\Lambda$ (the most common case is $V=\mathbb{R}^{r}$, whose underlying lattice, if not specified otherwise, is $\mathbb{Z}^{r}$ ). The top-dimensional polyhedra in $X$ are called facets, the codimension 1 polyhedra are called ridges. Balanced means that, for each ridge $\tau \in X$, the following balancing condition at $\tau$ is satisfied: the weighted sum of the primitive vectors of the facets $\sigma$ around $\tau$

$$
\sum_{\sigma \in X_{\tau<\sigma}^{(\operatorname{dim}(X))}} \omega(\sigma) v_{\sigma / \tau}
$$

vanishes "modulo $\tau$," or, precisely, lies in the linear vector space spanned by $\tau$, denoted by $V_{\tau}$. Here, $\omega(\sigma)$ denotes the weight of a facet $\sigma$, and a primitive vector $v_{\sigma / \tau}$ of $\sigma$ modulo $\tau$ is a vector in $\Lambda$ that points from $\tau$ towards $\sigma$ and fulfils the primitive condition: the lattice $\mathbb{Z} v_{\sigma / \tau}+\left(V_{\tau} \cap \Lambda\right)$ must be equal to the lattice $V_{\sigma} \cap \Lambda$. Slightly 
differently, in [1], the class of $v_{\sigma / \tau}$ modulo $V_{\tau}$ is called the primitive vector and $v_{\sigma / \tau}$ is just a representative of it. We will abbreviate the lattice $V_{\sigma} \cap \Lambda$ by $\Lambda_{\sigma}$.

The support of $X$, denoted by $X$, is the union of all facets in $X$ with non-zero weight. We call $X$ irreducible if, for any cycle $Y$ of the same dimension with $|Y| \subseteq|X|$, there exists an integer $\mu \in \mathbb{Z}$ such that $Y=\mu \cdot X$. The positive part of $X$, denoted by $X^{+}$, is the set of all faces contained in a facet with positive weight. A general element $x$ of $X$ is an element $x \in|X|$ that lies in the interior of a facet. If the underlying polyhedral complex is a fan (i.e., if all polyhedra are actually cones with vertex in 0 ), we call $X$ a fan cycle (or sometimes just fan).

In fact, given a cycle $X$, we do not really want to fix its structure as a polyhedral complex but only its support and its weights. Therefore, by abuse of notation, a cycle $X$ also denotes the class of balanced polyhedral complexes with the same support and agreeing weights (on the common refinement).

A (non-zero) rational function on $X$ is a function $\varphi:|X| \rightarrow \mathbb{R}$ that is integer affine on each polyhedron. Here, integer linear means that it maps lattice elements to integers, and integer affine means that it is a sum of an integer linear function (called the linear part) and a real constant. If $X$ is a fan, we also assume $\varphi(0)=0$. The divisor of $\varphi$, denoted by $\operatorname{div}(\varphi)=\varphi \cdot X$, is the balanced subcomplex of $X$ constructed in [1, subsection 3.3], namely, the codimension 1 skeleton $X \backslash X^{(\operatorname{dim} X)}$ together with the weights $\omega_{\varphi \cdot X}(\tau)$ for each ridge $\tau \in X$. These weights are given by the formula:

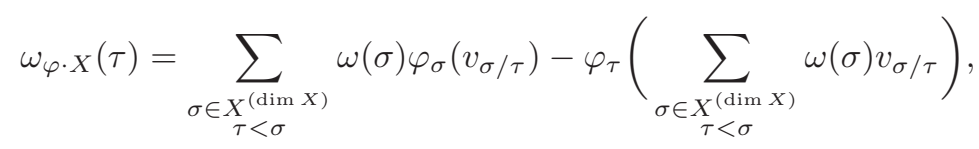

where $\varphi_{\sigma}: V_{\sigma} \rightarrow \mathbb{R}$ denotes the linear part of the affine function $\left.\varphi\right|_{\sigma}$. Note that the balancing condition of $X$ around $\tau$ ensures that the argument of $\varphi_{\tau}$ is indeed an element of $V_{\tau}$. Essentially, this weight measures the change of slope of $\varphi$ when traversing the ridge $\tau$, as illustrated in the following picture. 

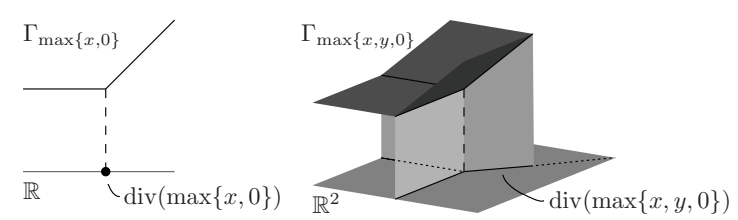

To be more precise, let $\Gamma_{\varphi}$ be the graph of $\varphi$ in $X \times \mathbb{R}$. It is a polyhedral complex whose polyhedra are in one-to-one correspondence with those of $X$, but in general $\Gamma_{\varphi}$ is not balanced. However, it can be completed to a cycle by adding facets in the $(0,-1)$-direction at each ridge of $\Gamma_{\varphi}$, equipped with the above weights. Now, if we (imaginary) intersect this tropically completed graph of $\varphi$ with $X \times\{-\infty\}$ (i.e., compute the tropical zero locus of $\varphi$ ), we obtain the cycle $\operatorname{div}(\varphi)=\varphi \cdot X$ of our definition.

If $\varphi$ is globally affine (respectively, linear), all weights are zero, which we denote by $\varphi \cdot X=0$. Let the support of $\varphi$, denoted by $|\varphi|$, be the subcomplex of $X$ containing the points $x \in|X|$ where $\varphi$ is not locally affine. Then we have $|\varphi \cdot X| \subseteq|\varphi|$. Furthermore, the intersection product is bilinear (see [1, subsection 3.6]). As the restriction of a rational function to a subcycle is again a rational function, we can also form multiple intersection products $\varphi_{1} \cdot \ldots \cdot \varphi_{l} \cdot X$. In this case, we will sometimes omit ". $X$ " to keep formulas shorter. Note that multiple intersection products are commutative (see $[\mathbf{1}$, subsection 3.7]).

A morphism of cycles $X \subseteq V=\Lambda \otimes \mathbb{R}$ and $Y \subseteq V^{\prime}=\Lambda^{\prime} \otimes \mathbb{R}$ is a map $f:|X| \rightarrow|Y|$ that is induced by a linear map $b \Lambda \rightarrow \Lambda^{\prime}$ and that maps each polyhedron of $X$ into a polyhedron of $Y$. We call $f$ an isomorphism and write $X \cong Y$, if there exists an inverse morphism and if, for all facets $\sigma \in X$, we have $\omega_{X}(\sigma)=\omega_{Y}(f(\sigma))$.

Such a morphism pulls back rational functions $\varphi$ on $Y$ to rational functions $f^{*}(\varphi)=\varphi \circ f$ on $X$. Note that the second condition of a morphism makes sure that we do not have to refine $X$ further. $f^{*}(\varphi)$ is already affine on each cone. The inclusion $\left|f^{*}(\varphi)\right| \subseteq f^{-1}(|\varphi|)$ holds, as the composition of an affine and a linear function is again affine.

Furthermore, we can push forward subcycles $Z$ of $X$ to subcycles $f_{*}(Z)$ of $Y$ of the same dimension. This is due to $[8,2.24,2.25]$ in the case of fans and can be generalized to complexes (see [1, subsection 
7.3]). We can omit further refinements here if we assume that $f(\sigma) \in Y$ for all $\sigma \in X$. Then $f_{*}(Z)$ is defined by assigning the following weights to the $\operatorname{dim}(Z)$-dimensional polyhedra $\sigma^{\prime} \in Y$ :

$$
\omega_{f_{*}(Z)}\left(\sigma^{\prime}\right)=\sum_{\substack{\sigma \in X \\ f(\sigma)=\sigma^{\prime}}}\left|\Lambda_{\sigma^{\prime}} / f\left(\Lambda_{\sigma}\right)\right| \cdot \omega_{Z}(\sigma) .
$$

By definition, we have $\left|f_{*}(Z)\right| \subseteq f(|Z|)$. The projection formula (see $[\mathbf{1}$, subsection 4.8]) connects all the above constructions via

$$
f_{*}\left(f^{*}(\varphi) \cdot X\right)=\varphi \cdot f_{*}(X) .
$$

By [1, Definition 9.3], it is also possible to form the intersection product of two cycles $X, Y$ in $V=\Lambda \otimes \mathbb{R}$ : we choose coordinates $x_{1}, \ldots, x_{r}$ on $\Lambda$ (and denote the same coordinates on the second factor of $V \times V$ by $\left.y_{1}, \ldots, y_{r}\right)$. Then the diagonal $\Delta$ in $V \times V$ is given by $\Delta=\max \left\{x_{1}, y_{1}\right\} \cdots \max \left\{x_{r}, y_{r}\right\} \cdot(V \times V)$. Furthermore, we consider the function $\pi: \Delta \rightarrow V,(x, x) \mapsto x$. Then the intersection product of $X$ and $Y$ in $V$ is given by

$$
X \cdot Y:=\pi_{*}\left(\max \left\{x_{1}, y_{1}\right\} \cdots \max \left\{x_{r}, y_{r}\right\} \cdot(X \times Y)\right) .
$$

This intersection product is independent of the chosen coordinates, commutative, associative, bilinear, admits the identity element $V$ and satisfies $(\varphi \cdot X) \cdot Y=\varphi \cdot(X \cdot Y)$, where $\varphi$ is a rational function on $X$.

Let us now turn to the concept of rational equivalence (we summarize [2]). Let $X$ be a zero-dimensional cycle. Then the degree $\operatorname{deg}(X)$ of $X$ denotes the sum of the weights of all points in $X$. Now let $X$ be an arbitrary cycle, and let $\varphi, \widetilde{\varphi}$ be two rational functions on $X$. We call them (rationally) equivalent if $\varphi-\widetilde{\varphi}$ is the sum of a bounded and a globally linear function. Obviously, this property is preserved when pulled back. Furthermore, if $Y$ is a one-dimensional subcycle of $X$, then $\operatorname{deg}(\varphi \cdot Y)=\operatorname{deg}(\widetilde{\varphi} \cdot Y)$ holds (see [1, Lemma 8.3]).

Let $X$ be a cycle, and let $Y$ be a subcycle. We call $Y$ rationally equivalent to zero, denoted by $Y \sim 0$, if there exists a morphism $f: X^{\prime} \rightarrow X$ and a bounded rational function $\phi$ on $X^{\prime}$ such that

$$
f_{*}\left(\phi \cdot X^{\prime}\right)=Y .
$$


This property commutes with taking Cartesian products, intersection products (of functions as well as of cycles) and with pushing forward. Moreover, if $Y$ is zero-dimensional, then $Y \sim 0 \operatorname{implies} \operatorname{deg}(Y)=0$.

Let $\widetilde{Y}$ be another subcycle of $X$. Then we call $Y$ and $\tilde{Y}$ rationally equivalent if $Y-\widetilde{Y}$ is rationally equivalent to zero. The easiest example of rationally equivalent cycles are translations. Let $X$ be a cycle in $V=\Lambda \otimes \mathbb{R}$, and let us denote by $X+v$ the translation of $X$ by an arbitrary vector $v \in V$. Then

$$
X \sim X+v
$$

holds (see also [17, Lemma 2.1]).

If $X, Y$ live in $V=\Lambda \otimes \mathbb{R}$, we call them numerically equivalent if, for any cycle $Z$ in $V$ of complementary dimension, the equation

$$
\operatorname{deg}(X \cdot Z)=\operatorname{deg}(Y \cdot Z)
$$

holds.

Let $X$ be a cycle in $V=\Lambda \otimes \mathbb{R}$. We define the degree or recession fan of $X$, denoted by $\delta(X)$, as follows: $\delta(X)$ is supported on the purely $\operatorname{dim}(X)$-dimensional part of the polyhedral set

$$
\bigcup_{\sigma \in X} \operatorname{rc}(\sigma)
$$

Here, the recession cone $\operatorname{rc}(\sigma)$ of a polyhedron $\sigma$ is defined to be the cone containing all vectors $v \in V$ such that, starting at an arbitrary point $x \in \sigma$, the ray $x+\mathbb{R} v$ is contained in $\sigma$. Now, for a fine enough fan structure on this polyhedral set, the weights are given by

$$
\omega_{\delta(X)}\left(\sigma^{\prime}\right):=\sum_{\substack{\sigma \in X \\ \sigma^{\prime} \subseteq \operatorname{rc}(\sigma)}} \omega_{X}(\sigma) .
$$

In particular, if $X$ is a curve, then $\delta(X)$ is just the union of all unbounded rays in $X$ and the weights are the sums of the weights of the rays in $X$ of a given direction. Geometrically, we simply shrink all bounded parts of $X$ to a point and move the final single vertex to the origin. 
The main result of [2] is that, for cycles $X$ in $V=\Lambda \otimes \mathbb{R}$, rational equivalence, numerical equivalence and "having the same degree" coincides. To prove this, an important substep is to show that $X$ is always rationally equivalent to its degree,

$$
X \sim \delta(X) .
$$

1.2. Local computation of intersection products. Let $X$ be a cycle, and let $\tau \in X$ be a polyhedron in $X$. We define the star of $X$ at $\tau$ to be the fan

$$
\operatorname{Star}_{X}(\tau):=\{\bar{\sigma} \mid \tau<\sigma \in X\},
$$

where $\bar{\sigma}$ denotes the cone in $V / V_{\tau}$ spanned by the image of $\sigma-\tau$ under the quotient map $q: V \rightarrow V / V_{\tau}$. We make it into a cycle by defining $\omega_{\operatorname{Star}_{X}(\tau)}(\bar{\sigma})=\omega_{X}(\sigma)$ for all facets $\bar{\sigma}$ of $\operatorname{Star}_{X}(\tau)$ (note that $q$ preserves the codimension of the polyhedra). This fan contains all the local information of $X$ around $\tau$ and can be considered as the tropical version of a small neighborhood of an interior point of $\tau$ (divided by the linearity space $V_{\tau}$ ). Its dimension equals the codimension of $\tau$ in $X$.

Furthermore, let $\varphi$ be a rational function on $X$. Choose an arbitrary affine function $\psi$ with $\left.\varphi\right|_{\tau}=\left.\psi\right|_{\tau}$. Then $\varphi-\psi$ induces a rational function on $\operatorname{Star}_{X}(\tau)$ which we denote by $\varphi^{\tau}$ (and call it a germ of $\varphi$ at $\tau$ ). This function is only unique up to adding a linear function, which is enough for us as it does not change its divisor. The following proposition shows that our intersection products are local constructions (i.e., can be expressed in terms of stars and germs).

Proposition 1.1. Let $X$ be a cycle with polyhedra $\tau<\sigma \in X$. Let $\varphi, \varphi_{1}, \ldots, \varphi_{l}$ be rational functions on $X$. Then the following statements are true:

(i) $\operatorname{Star}_{\operatorname{Star} X(\tau)}(\bar{\sigma})=\operatorname{Star}_{X}(\sigma)$.

(ii) $\left(\varphi^{\tau}\right)^{\sigma}=\varphi^{\sigma}$ on $\operatorname{Star}_{X}(\sigma)$ (up to adding a linear function).

(iii) $\operatorname{Star}_{\varphi \cdot X}(\tau)=\varphi^{\tau} \cdot \operatorname{Star}_{X}(\tau)$.

(iv) $\operatorname{Star}_{\varphi_{1} \cdot \ldots \cdot \varphi_{l} \cdot X}(\tau)=\varphi_{1}^{\tau} \cdot \ldots \cdot \varphi_{l}^{\tau} \cdot \operatorname{Star}_{X}(\tau)$.

(v) If $l=\operatorname{dim}(X)-\operatorname{dim}(\tau)$, then

$$
\omega_{\varphi_{1} \cdot \ldots \cdot \varphi_{l} \cdot X}(\tau)=\omega_{\varphi_{1}^{\tau} \cdot \ldots \cdot \varphi_{l}^{\tau} \cdot \operatorname{Star} X(\tau)}(\{0\}),
$$


i.e., we can compute the weight of $\tau$ in $\varphi_{1} \cdot \ldots \cdot \varphi_{l} \cdot X$ "locally" in $\operatorname{Star}_{X}(\tau)$.

Proof. Items (i) and (ii) are immediate consequences of the definitions. Item (iv) follows from (iii) by induction and (v) is just a special case of (iv), namely, when $\varphi_{1}^{\tau} \cdot \ldots \cdot \varphi_{l}^{\tau} \cdot \operatorname{Star}_{X}(\tau)$ is zero-dimensional. Hence, we are left with (iii).

Let $r:=\operatorname{dim}(X)-\operatorname{dim}(\tau)$ be the codimension of $\tau$ in $X$. The statement is trivial when $r=0$ : Both sides are 0 . Assume $r=1$. In this case, we only have to check

$$
\omega_{\varphi \cdot X}(\tau)=\omega_{\varphi^{\tau} \cdot \operatorname{Star}_{X}(\tau)}(\{0\}) .
$$

By adding an affine function we can assume that $\left.\varphi\right|_{\tau}=0$ without changing the intersection product and, in particular, the weight of $\tau$ in $\varphi \cdot X$. But then we can replace both weights according to their definition and observe that

$$
\begin{aligned}
\omega_{\varphi \cdot X}(\tau) & =\sum_{\sigma \in X_{\tau<\sigma}^{(\operatorname{dim}(X))}} \omega(\sigma) \varphi_{\sigma}\left(v_{\sigma / \tau}\right) \\
& =\sum_{\bar{\sigma} \in \operatorname{Star}_{X}(\tau)^{(1)}} \omega(\bar{\sigma}) \varphi^{\tau}\left(v_{\bar{\sigma} /\{0\}}\right)=\omega_{\varphi^{\tau} \cdot \operatorname{Star}_{X}(\tau)}(\{0\})
\end{aligned}
$$

holds true, as $\left[v_{\sigma / \tau}\right]=v_{\bar{\sigma} /\{0\}} \in V / V_{\tau}$.

Now let us assume $r>1$, and let $\tau^{\prime}$ be a ridge in $X$. Then we can use the previous case as well as (i) and (ii) and obtain

$$
\begin{aligned}
& \omega_{\varphi \cdot X}\left(\tau^{\prime}\right) \stackrel{r \equiv 1}{=} \omega_{\varphi^{\tau^{\prime}} \cdot \operatorname{Star}_{X}\left(\tau^{\prime}\right)}(\{0\}) \\
& \stackrel{(\mathrm{a}),(\mathrm{b})}{=} \omega_{\left(\varphi^{\tau}\right) \tau^{\prime} \cdot \operatorname{Star}_{\operatorname{Star}_{X}(\tau)}\left(\tau^{\prime}\right)}(\{0\}) \\
& \stackrel{r \equiv 1}{=} \omega_{\varphi^{\tau} \cdot \operatorname{Star}_{X}(\tau)}\left(\bar{\tau}^{\prime}\right) \text {, }
\end{aligned}
$$

which proves the claim.

We can extend this to the case of the intersection product of two cycles. 
Proposition 1.2. Let $X$ and $Y$ be two cycles in $V=\mathbb{R} \otimes \Lambda$. Then the equation

$$
\operatorname{Star}_{X \cdot Y}(\tau)=\operatorname{Star}_{X}(\tau) \cdot \operatorname{Star}_{Y}(\tau)
$$

holds for all polyhedra $\tau \in X \cdot Y$.

Proof. First, we fix some notation. Let $x_{1}, \ldots, x_{r}$ be a lattice basis of $\Lambda^{\vee}$ such that the first $d:=\operatorname{codim}_{V}(\tau)$ elements generate $V_{\tau}^{\perp}$. When we consider the product $\Lambda \times \Lambda$, the same coordinates on the second factor will be denoted by $y_{1}, \ldots, y_{r}$. Furthermore, let $\Delta: V \rightarrow V \times V$, $x \mapsto(x, x)$ denote the diagonal map. By definition of the intersection product of cycles and using Proposition 1.1 (iv) we have to compute

$$
\begin{aligned}
\operatorname{Star}_{\max }\left\{x_{1}, y_{1}\right\} \cdots \max \left\{x_{r}, y_{r}\right\} \cdot(X \times Y) & (\Delta(\tau)) \\
& =\max \left\{x_{1}, y_{1}\right\} \cdots \max \left\{x_{r}, y_{r}\right\} \cdot \operatorname{Star}_{X \times Y}(\Delta(\tau))
\end{aligned}
$$

and

$$
\max \left\{x_{1}, y_{1}\right\} \cdots \max \left\{x_{d}, y_{d}\right\} \cdot\left(\operatorname{Star}_{X}(\tau) \times \operatorname{Star}_{Y}(\tau)\right)
$$

respectively. Thus, the statement follows from the fact that

$$
\begin{aligned}
\max \left\{x_{d+1}, y_{d+1}\right\} & \cdots \max \left\{x_{r}, y_{r}\right\} \\
\cdot\left(V \times V / \Delta\left(V_{\tau}\right)\right) & \longrightarrow V / V_{\tau} \times V / V_{\tau}, \\
(x, y) & \longmapsto(x, y)
\end{aligned}
$$

is an isomorphism and can be restricted to an isomorphism of

$$
\max \left\{x_{d+1}, y_{d+1}\right\} \cdots \max \left\{x_{r}, y_{r}\right\} \cdot \operatorname{Star}_{X \times Y}(\Delta(\tau))
$$

and $\operatorname{Star}_{X}(\tau) \times \operatorname{Star}_{Y}(\tau)$

1.3. Transversal intersections. Let us now consider "generic" intersections.

Definition 1.3. Let $X$ and $Y$ be two cycles in $V=\Lambda \otimes \mathbb{R}$ of codimension $c$, respectively $d$. We say $X$ and $Y$ intersect transversally if $X \cap Y$ is of pure codimension $c+d$ and if, for each facet $\tau$ in $X \cap Y$, the corresponding neighborhoods $\operatorname{Star}_{X}(\tau)$ and $\operatorname{Star}_{Y}(\tau)$ are (transversal) affine subspaces of $V$. 
In this case, by locality of the intersection product, the computation of $X \cdot Y$ can be reduced to the intersection of vector spaces. This motivates the following study of intersections of linear functions and spaces.

Lemma 1.4. Let $h_{1}, \ldots, h_{l}$ be integer linear functions on $V(l \leq$ $\operatorname{dim}(V)=: r)$, and define the rational functions $\varphi_{i}:=\max \left\{h_{i}, 0\right\}$ on $V$. Let $H: V \rightarrow \mathbb{R}^{l}$ be the linear function with $H(x)=\left(h_{1}(x), \ldots\right.$, $\left.h_{l}(x)\right)$ and let us assume that $H$ has full rank. Then $\varphi_{1} \cdot \ldots \cdot \varphi_{l} \cdot V$ is equal to the subspace $\operatorname{ker}(H)$ with weight $\operatorname{ind}(H):=\left|\mathbb{Z}^{l} / H(\Lambda)\right|$. Here we give $V$ the fan structure consisting of all cones where each of the $h_{i}$ is either positive or zero or negative, with all weights being 1.

Proof. Let us assume $l=1$ first (i.e., $H=h_{1}$ ). In this case, we have to compute the weight of the only ridge in $V$ which is $h_{1}^{\perp}=\operatorname{ker}(H)$. This ridge is contained in the two facets corresponding to $h_{i} \geq 0$ and $h_{i} \leq 0$. Let $v_{\geq}=-v_{\leq}$be corresponding primitive vectors. This implies that, for example, $v_{\geq}$generates the one-dimensional lattice $\Lambda / h_{1}^{\perp} \cong h_{1}(\Lambda)$, and therefore, $\left|\mathbb{Z} / h_{1}(\Lambda)\right|=h_{1}\left(v_{\geq}\right)$. On the other hand, we can compute the weight of $h_{1}^{\perp}$ in $h_{1} \cdot V$ to be

$$
\omega_{h_{1} \cdot V}\left(h_{1}^{\perp}\right)=\varphi_{1}\left(v_{\geq}\right)+\varphi_{1}\left(v_{\leq}\right)=h_{1}\left(v_{\geq}\right)+0=\left|\mathbb{Z} / h_{1}(\Lambda)\right| .
$$

Now we make induction for $l>1$. The induction hypothesis says that $\varphi_{2} \cdot \ldots \varphi_{l} \cdot V$ is equal to the subspace $\operatorname{ker}\left(H^{\prime}\right)$ with weight $\operatorname{ind}\left(H^{\prime}\right)$, where $H^{\prime}=h_{2} \times \cdots \times h_{l}$. By applying the case $l=1$ to the vector space $\operatorname{ker}\left(H^{\prime}\right)=\left(\operatorname{ker}\left(H^{\prime}\right) \cap \mathbb{Z}^{r}\right) \otimes \mathbb{R}$, we obtain that $\varphi_{1} \cdot \ldots \varphi_{l} \cdot V$ is equal to the subspace $h_{1}^{\perp} \cap \operatorname{ker}\left(H^{\prime}\right)=\operatorname{ker}(H)$ with weight $\operatorname{ind}\left(\left.h_{1}\right|_{\operatorname{ker}\left(H^{\prime}\right)}\right) \cdot \operatorname{ind}\left(H^{\prime}\right)$. We have to show that this weight coincides with $\operatorname{ind}(H)$. This follows from the exact sequence

$$
\begin{aligned}
& 0 \rightarrow h_{1}\left(\operatorname{ker}\left(H^{\prime}\right) \cap \Lambda\right) \rightarrow H(\Lambda) \rightarrow H^{\prime}(\Lambda) \rightarrow 0 \\
& h_{1}(x) \quad \mapsto H(x)=\left(h_{1}(x), 0\right) \\
& H(x) \quad \mapsto \quad H^{\prime}(x)
\end{aligned}
$$

and its induced quotient sequence

$$
0 \longrightarrow \mathbb{Z}^{l-1} / H^{\prime}(\Lambda) \longrightarrow \mathbb{Z}^{l} / H(\Lambda) \longrightarrow \mathbb{Z} / h_{1}\left(\operatorname{ker}\left(H^{\prime}\right) \cap \Lambda\right) \longrightarrow 0
$$


Remark 1.5. In the special case $l=r$ the weight of $\{0\}$ in the intersection product $\varphi_{1} \cdot \ldots \cdot \varphi_{r} \cdot V$ is $\left|\mathbb{Z}^{r} / H(\Lambda)\right|$, which equals $|\operatorname{det}(M)|$ where $M$ is a matrix representation of $H$ with respect to a lattice basis of $\Lambda$ and the standard basis of $\mathbb{Z}^{r}$. This special case of the statement is proven in [17, Lemma 5.1]. Note that, in this case, if $\operatorname{det}(M)$ is zero, the intersection product is zero as well. Hence, this version can be extended to the case where $H$ does not have full rank.

Now we use this lemma to compute the intersection of two linear subspaces.

Lemma 1.6. Let $U$ and $W$ be two subspaces of $V=\mathbb{R} \otimes \Lambda$ (with rational slope) such that $U+W=V$. If we consider $U$ and $W$ as cycles with weight 1 , their intersection product can be computed to be:

$$
U \cdot W=\left|\Lambda /\left(\Lambda_{U}+\Lambda_{W}\right)\right| \cdot(U \cap W) .
$$

Proof. By definition, we have to compute

$$
\max \left\{x_{1}, y_{1}\right\} \cdots \max \left\{x_{r}, y_{r}\right\} \cdot(U \times W),
$$

(where we chose arbitrary coordinates on $\Lambda$ ). Instead of $\max \left\{x_{i}, y_{i}\right\}$, we can as well subtract the linear function $y_{i}$ and use the functions $\max \left\{x_{i}-y_{i}, 0\right\}$. Now we can apply Lemma 1.4. In our case, the function $H$ is just

$$
\begin{aligned}
H: \Lambda \times \Lambda & \longrightarrow \Lambda, \\
(x, y) & \longmapsto x-y .
\end{aligned}
$$

Restricted to $U \times W$, this provides

$$
U \cdot W=\left|\Lambda / H\left(\Lambda_{U} \times \Lambda_{W}\right)\right| \cdot \pi_{*}(\operatorname{ker}(H))=\left|\Lambda /\left(\Lambda_{U} \mp \Lambda_{W}\right)\right| \cdot(U \cap W) .
$$

Now, as a combination of Proposition 1.2 and Lemma 1.6, we obtain the following result.

Corollary 1.7. Let $X$ and $Y$ be two cycles in $V=\mathbb{R} \otimes \Lambda$ that intersect transversally. Then $X \cdot Y=\left(X \cap Y, \omega_{X \cap Y}\right)$ with the following weight function. Any facet $\tau$ in $X \cap Y$ is the intersection of two facets $\sigma, \sigma^{\prime}$ 
in $X$, respectively $Y$. Then the weight of $\tau=\sigma \cap \sigma^{\prime}$ is

$$
\omega_{X \cap Y}\left(\sigma \cap \sigma^{\prime}\right)=\omega_{X}(\sigma) \omega_{Y}\left(\sigma^{\prime}\right)\left|\Lambda / \Lambda_{\sigma}+\Lambda_{\sigma^{\prime}}\right| .
$$

1.4. Comparison to the fan displacement rule. In [7], the authors introduce Minkowski weights to describe the Chow cohomology groups of a toric variety combinatorially. Moreover, they compute the cup-product of these cohomology groups in terms of Minkowski weights. In this subsection, we show explicitly that, when we interpret Minkowski weights as tropical cycles, this cup-product coincides with our product of tropical cycles. Another approach to this topic is given in [12, Section 9] and [13].

Let $\Theta$ be a complete fan in a vector space $V=\mathbb{R} \otimes \Lambda$ of dimension $r$ (in [7], the fan is called $\Delta$ and the lattice is called $N$ ). Let $\Theta^{(k)}$ denote the set of $k$-dimensional cones in $\Theta$ (in [7], the exponent indicates the codimension, i.e., $\Delta^{(k)}$ means $\left.\Theta^{(r-k)}\right)$.

Definition 1.8. (cf., [7, Section 2]). A Minkowski weight c of codimension $k$ is an integer-valued function on $\Theta^{(r-k)}$ that satisfies, for any $\tau \in \Theta^{(r-k-1)}$,

$$
\sum_{\substack{\sigma \in \Theta^{(r-k)} \\ \tau \subseteq \sigma}} c(\sigma) v_{\sigma / \tau} \in \Lambda_{\tau}
$$

(in [7], primitive vectors are denoted by $n_{\sigma, \tau}$ ).

Let $c$ be a Minkowski weight of codimension $k$. Of course, if we set $X(c)$ to be the fan $\bigcup_{0 \leq i \leq r-k} \Theta^{(i)}$ with weight function $c$, the Minkowski weight condition precisely coincides with our balancing condition, i.e., $X(c)$ is a tropical cycle of codimension $k$.

In [7], it is shown that Minkowski weights are in one-to-one correspondence with the Chow cohomology classes of the toric variety associated to the fan $\Theta$ and therefore admit a cup-product with the following properties. Let $c, c^{\prime}$ be Minkowski weights of codimension $k, k^{\prime}$. Then the cup-product $c \cup c^{\prime}$ is a Minkowski weight of codimen- 
sion $k+k^{\prime}$ given by

$$
\left(c \cup c^{\prime}\right)(\tau)=\sum_{\substack{\sigma \in \Theta^{r-k} \\ \sigma^{\prime} \in \Theta^{r-k^{\prime}} \\ \tau \subseteq \sigma, \sigma^{\prime}}} m_{\sigma, \sigma^{\prime}}^{\tau} \cdot c(\sigma) \cdot c^{\prime}\left(\sigma^{\prime}\right) .
$$

Here, the coefficients are not unique but depend on the choice of a generic vector $v \in V$. If we fix such a vector $v$, then

$$
m_{\sigma, \sigma^{\prime}}^{\tau}= \begin{cases}\left|\Lambda / \Lambda_{\sigma}+\Lambda_{\sigma^{\prime}}\right| & \text { if }(\sigma+v) \cap \sigma^{\prime} \neq \emptyset, \\ 0 & \text { otherwise }\end{cases}
$$

(cf., [7, Introduction]). With the tools developed in the previous sections, we can show easily (and purely tropically) that the cupproduct of Minkowski weights coincides with our intersection product of tropical cycles in $V$. An independent proof of this statement is given in [13, Theorem 4.4].

Theorem 1.9. Let $c$ and $c^{\prime}$ be Minkowski weights of codimension $k, k^{\prime}$. Then the following equation holds:

$$
X(c) \cdot X\left(c^{\prime}\right)=X\left(c \cup c^{\prime}\right) .
$$

Proof. For each facet $\tau$ in $X\left(c \cup c^{\prime}\right)$, we have to show

$$
\omega_{X(c) \cdot X\left(c^{\prime}\right)}(\tau)=\left(c \cup c^{\prime}\right)(\tau) .
$$

First, note that we can compute both sides locally on $\operatorname{Star}_{\Theta}(\tau)$, where we of course define the "local" Minkowski weights by $\bar{c}(\bar{\sigma}):=c(\sigma)$ and $\bar{c}^{\prime}\left(\bar{\sigma}^{\prime}\right):=c^{\prime}\left(\sigma^{\prime}\right)$. For the left hand side, this follows from Lemma 1.2 and for the right hand side, it follows from

$$
\left|\Lambda / \Lambda_{\sigma}+\Lambda_{\sigma^{\prime}}\right|=\left|\left(\Lambda / \Lambda_{\tau}\right) /\left(\left(\Lambda_{\sigma}+\Lambda_{\sigma^{\prime}}\right) / \Lambda_{\tau}\right)\right|
$$

Therefore, we can assume $k+k^{\prime}=r$ and $\tau=\{0\}$. In this case, by plugging in the definition on the right hand side and choosing a generic vector $v \in V$, it remains to show

$$
\operatorname{deg}\left(X(c) \cdot X\left(c^{\prime}\right)\right)=\sum_{\substack{\sigma \in \Theta^{r-k} \\ \sigma^{\prime} \in \Theta^{r-k^{\prime}} \\(\sigma+v) \cap \sigma^{\prime} \neq \emptyset}}\left|\Lambda / \Lambda_{\sigma}+\Lambda_{\sigma^{\prime}}\right| \cdot c(\sigma) \cdot c^{\prime}\left(\sigma^{\prime}\right) .
$$


Now, for a generic vector $v \in V$, we can assume that $X(c)+v$ and $X\left(c^{\prime}\right)$ intersect transversally (in fact, this is what the authors of [7] mean by a generic vector). Note that, in fact, the sum on the right hand side runs through all points in the intersection of $X(c)+v$ and $X\left(c^{\prime}\right)$. Therefore, by Corollary 1.7, it equals $\operatorname{deg}\left((X(c)+v) \cdot X\left(c^{\prime}\right)\right)$. But as $X(c)+v$ and $X(c)$ are rationally equivalent, the equation $\operatorname{deg}\left(X(c) \cdot X\left(c^{\prime}\right)\right)=\operatorname{deg}\left((X(c)+v) \cdot X\left(c^{\prime}\right)\right)$ holds, and the statement follows.

1.5. Convexity and positivity. A non-zero cycle $X$ is called positive, denoted $X>0$, if all weights are non-negative. By throwing away the facets with weight 0 (and all polyhedra contained in only such facets) we can assume all weights to be positive. A rational function $\varphi$ on $X$ is called convex if it is locally the restriction of a convex function on $V$. The pull-back $f^{*}(\varphi)$ of a convex function is again convex, as the composition of a convex function and a linear map is again convex. Moreover, if $Z$ is a subcycle of $X$, then $\left.\varphi\right|_{|Z|}$ is also convex on $Z$. Combining positivity and convexity, we get the following result.

Proposition 1.10. Let $X$ be a positive cycle, and let $\varphi$ be a convex function on $X$. Then:

(i) $\varphi \cdot X$ is positive,

and

(ii) $|\varphi|=|\varphi \cdot X|$.

Proof. First of all, note that we can assume that $X$ is a onedimensional fan, as all intersection weights can be computed locally modulo the ridge (cf., Lemma 1.1 (iii)) and convexity is preserved when adding linear functions or when considering the function induced on the quotient. Thus, we assume that $X=\left\{\{0\}, \rho_{1}, \ldots, \rho_{r}\right\}$ is a onedimensional fan with positive weights $\omega\left(\rho_{i}\right)>0$. The statements of the lemma translate to:

(i) $\varphi$ convex $\Rightarrow \varphi \cdot X>0$,

(ii) $\varphi$ convex, $\varphi \cdot X=0 \Rightarrow \varphi$ linear. 
We use the following criteria for linearity and convexity. Let $\varphi$ be a rational function on $X$, and let us abbreviate the primitive vector of the ray $\rho_{i}$ by $v_{i}$. Then

(i) $\varphi$ is linear if, and only if, for all $\lambda_{1}, \ldots, \lambda_{r} \in \mathbb{R}$ with $\sum_{i} \lambda_{i} v_{i}=0$, the following holds:

$$
\sum_{i} \lambda_{i} \varphi\left(v_{i}\right)=0
$$

(ii) $\varphi$ is convex if, and only if, for all positive $\lambda_{1}, \ldots, \lambda_{r} \geq 0$ with $\sum_{i} \lambda_{i} v_{i}=0$, the following holds:

$$
\sum_{i} \lambda_{i} \varphi\left(v_{i}\right) \geq 0
$$

Now let $\varphi$ be convex. We can apply criterion (ii) to the coefficients $\omega\left(\rho_{i}\right)$, which are positive and satisfy $\sum_{i} \omega\left(\rho_{i}\right) v_{i}=0$. This provides

$$
\omega_{\varphi \cdot X}(\{0\})=\sum_{i} \omega\left(\rho_{i}\right) \varphi\left(v_{i}\right) \geq 0,
$$

which proves (i).

For (ii), let us assume that $\sum_{i} \omega\left(\rho_{i}\right) \varphi\left(v_{i}\right)=0$ (i.e., $\varphi \cdot X=0$ ) but $\varphi$ is not linear. Then, by (i), there exist $\lambda_{1}, \ldots, \lambda_{r}$ with $\sum_{i} \lambda_{i} v_{i}=0$ but $\sum_{i} \lambda_{i} \varphi\left(v_{i}\right) \neq 0$. Without loss of generality, we can assume $\sum_{i} \lambda_{i} \varphi\left(v_{i}\right)<0$ (otherwise, we replace $\lambda_{i}$ by $-\lambda_{i}$ ). For large enough $C \in \mathbb{R}$ the coefficients $\lambda_{i}^{\prime}:=\lambda_{i}+C \omega\left(\rho_{i}\right)$ are all positive and still satisfy $\sum_{i} \lambda_{i}^{\prime} v_{i}=0$ and $\sum_{i} \lambda_{i}^{\prime} \varphi\left(v_{i}\right)<0$, which contradicts (ii). Therefore, $\varphi$ is linear, which proves $(b)$.

The following application of this proposition will be useful for us later.

Proposition 1.11. Let $f: X \rightarrow Y$ be a morphism of cycles, and let us assume that $Y$ is positive. Let, furthermore, $\varphi_{1}, \ldots, \varphi_{l}$ denote convex functions on $Y$. Then the following equation of sets holds:

$$
\left|f^{*}\left(\varphi_{1}\right) \cdots f^{*}\left(\varphi_{l}\right) \cdot X\right| \subseteq f^{-1}\left(\left|\varphi_{1} \cdots \varphi_{l} \cdot Y\right|\right) .
$$


Proof. This can be proven by an easy induction. If $l=1$, we have

$$
\left|f^{*}\left(\varphi_{1}\right) \cdot X\right|=\left|f^{*}\left(\varphi_{1}\right)\right| \subseteq f^{-1}\left(\left|\varphi_{1}\right|\right)=f^{-1}\left(\left|\varphi_{1} \cdot Y\right|\right),
$$

where the equalities follow from Proposition 1.10 (a). Now, for arbitrary $l$, we can apply the case of a single function to $\varphi_{l}$, obtaining

$$
\left|f^{*}\left(\varphi_{l}\right) \cdot X\right| \subseteq f^{-1}\left(\left|\varphi_{l} \cdot Y\right|\right) .
$$

This shows that we can restrict the morphism $f$ to $f: f^{*}\left(\varphi_{l}\right) \cdot X \rightarrow$ $\varphi_{l} \cdot Y$. As $\varphi_{l} \cdot Y$ is still positive by Proposition 1.10 (b), we can apply the induction hypothesis to this restriction, which yields the result.

1.6. Complete intersections. We define the set of $m$-dimensional complete intersections $Z_{m}^{\text {c.i. }}(X) \subset Z_{m}(X)$ to be the set of $m$-dimensional cycles in $X$ obtained as an intersection product $\varphi_{1} \cdots \varphi_{l} \cdot X$ (where $l=\operatorname{dim}(X)-m)$.

Let $C, C^{\prime} \in Z_{*}^{\text {c.i. }}(X)$ be complete intersections given by $C=$ $\varphi_{1} \cdots \varphi_{l} \cdot X$ and $C^{\prime}=\varphi_{1}^{\prime} \cdots \varphi_{l^{\prime}}^{\prime} \cdot X$. Then we define

$$
C \cdot C^{\prime}:=\varphi_{1} \cdots \varphi_{l} \cdot \varphi_{1}^{\prime} \cdots \varphi_{l^{\prime}}^{\prime} \cdot X .
$$

Using commutativity of the intersection product of functions, this multiplication is independent of the chosen functions, commutative and satisfies $\left|C \cdot C^{\prime}\right|=|C| \cap\left|C^{\prime}\right|$. Note that, if $X=V=\Lambda \otimes \mathbb{R}$, it follows from [1, Corollary 9.8] that this definition coincides with the usual intersection product of cycles.

Let $C \in Z_{m}^{\text {c.i. }}(X)$ be given by $C=\varphi_{1} \cdots \varphi_{l} \cdot X$, and let $f: Y \rightarrow X$ be a tropical morphism. Then we would like to define the pull-back of $C$ along $f$ to be the complete intersection

$$
f^{*}(C):=f^{*}\left(\varphi_{1}\right) \cdots f^{*}\left(\varphi_{l}\right) \cdot Y .
$$

However, in general, this definition is not independent of the chosen functions $\varphi_{1}, \ldots, \varphi_{l}$. For us, it is enough to consider the case of projections where this indeterminacy does not occur.

Proposition 1.12. Let $X$ and $Y$ be two cycles, and let $\pi: X \times Y \rightarrow X$ be the projection onto the first factor. Moreover, let $Z$ be a complete intersection of $X \times Y$, and consider the map $f=\left.\pi\right|_{Z}: Z \rightarrow X$. Now, 
if $C=\varphi_{1} \cdots \varphi_{l} \cdot X$ is a complete intersection in $X$, then the pull-back

$$
f^{*}(C):=f^{*}\left(\varphi_{1}\right) \cdots f^{*}\left(\varphi_{l}\right) \cdot Z
$$

is well defined, and the equation

$$
\left|f^{*}(C)\right| \subseteq f^{-1}(|C|)
$$

holds.

Proof. First, we apply [1, subsection 9.6], which yields

$$
\pi^{*}\left(\varphi_{1}\right) \cdots \pi^{*}\left(\varphi_{l}\right) \cdot(X \times Y)=\left(\varphi_{1} \cdots \varphi_{l} \cdot X\right) \times Y=C \times Y .
$$

Therefore, $f^{*}\left(\varphi_{1}\right) \cdots f^{*}\left(\varphi_{l}\right) \cdot Z$ is just the product of the complete intersections $C \times Y$ and $Z$, which does not depend on any choices. Moreover, its support is contained in $|C \times Y|$, and the equation of sets follows.

Remark 1.13 (Pulling back preserves numerical equivalence). Let $C$ and $C^{\prime}$ be complete intersections in $\mathbb{R}^{r}$, and let $f: Y \rightarrow \mathbb{R}^{r}$ be a tropical morphism. Then, if $C$ and $C^{\prime}$ are numerically equivalent, also $f^{*}(C)$ and $f^{*}\left(C^{\prime}\right)$ are numerically equivalent in the following sense. If $Z$ is an arbitrary complete intersection in $Y$ of complementary dimension, then

$$
\operatorname{deg}\left(f^{*}(C) \cdot Z\right)=\operatorname{deg}\left(f^{*}\left(C^{\prime}\right) \cdot Z\right)
$$

holds. This follows from the projection formula,

$$
\operatorname{deg}\left(f^{*}(C) \cdot Z\right)=\operatorname{deg}\left(f_{*}\left(f^{*}(C) \cdot Z\right)\right)=\operatorname{deg}\left(C \cdot f_{*}(Z)\right) .
$$

In particular, if we move around $C$ in $V$, the numerical properties of the pull-backs of the original and the translated cycle coincide.

1.7. General position. We now investigate what can be said about the set-theoretic preimage of a general translation of a cycle under a morphism $f$. This section is a simple generalization of $[\mathbf{1 7}$, Section $3]$, where $f$ is (a combination of) evaluation morphisms.

Lemma 1.14. Let $X$ be a pure-dimensional polyhedral complex, and let $f: X \rightarrow \mathbb{R}^{r}$ be a morphism of polyhedral complexes (i.e., $f$ is 
linear on every polyhedron of $X)$. Furthermore, let $C$ be a polyhedral complex in $\mathbb{R}^{r}$ and consider the subcomplex $f^{-1}(C)$ of $X$ consisting of all polyhedra $\tau \cap f^{-1}(\gamma), \tau \in X, \gamma \in C$. Then for a general translation $C^{\prime}=C+v$ (i.e., $v \in \mathbb{R}^{r}$ can be chosen from an open dense subset of $\left.\mathbb{R}^{r}\right)$ the codimension of each non-empty polyhedron $\tau \cap f^{-1}(\gamma)$ of $X$ is equal to

$$
\operatorname{codim}_{X}\left(\tau \cap f^{-1}(\gamma)\right)=\operatorname{codim}_{X}(\tau)+\operatorname{codim}_{\mathbb{R}^{r}}(\gamma) .
$$

Proof. For each $\tau$ in $X$ and $\gamma$ in $C$, we consider the map

$$
f_{\tau}: \operatorname{Aff} \operatorname{Span}(\tau) \longrightarrow \mathbb{R}^{r}
$$

induced by $\left.f\right|_{\tau}$. Now we are interested in $\tau \cap f^{-1}\left(\gamma^{\prime}\right)=\tau \cap f_{\tau}^{-1}\left(\gamma^{\prime}\right)$ for general translations $\gamma^{\prime}$ of $\gamma$. We must distinguish the cases $\operatorname{Im}\left(f_{\tau}\right)+V_{\gamma}=\mathbb{R}^{r}$ and $\operatorname{Im}\left(f_{\tau}\right)+V_{\gamma} \neq \mathbb{R}^{r}$. In the latter case, $f_{\tau}^{-1}\left(\gamma^{\prime}\right)$ is empty for general $\gamma^{\prime}$. In the former case, $f_{\tau}^{-1}\left(\gamma^{\prime}\right)$ is a polyhedron of dimension $\operatorname{dim}(\tau)+\operatorname{dim}(\gamma)-r$, and for general $\gamma^{\prime}$, it is disjoint from $\tau$ or intersects the interior of $\tau$, in which case $\tau \cap f_{\tau}^{-1}\left(\gamma^{\prime}\right)$ has the same dimension $\operatorname{dim}(\tau)-\operatorname{codim}_{\mathbb{R}^{r}}(\gamma)$, which is the expected dimension.

As there are only finitely many pairs $\tau, \gamma$, this holds simultaneously for all pairs for general enough translations of $C$.

This technical statement has the following more applicable consequences.

Proposition 1.15 (Preimages of general translations). Let $f_{k}: X \rightarrow$ $\mathbb{R}^{r}, k=1, \ldots, n$ be morphisms of pure-dimensional polyhedral complexes, and let $C_{k}, k=1, \ldots, n$ be cycles in $\mathbb{R}^{r}$. Then, for a general translation $C_{k}^{\prime}=C_{k}+v_{k}, v_{k} \in \mathbb{R}^{r}$, the following holds. Either $Z:=f_{1}^{-1}\left(C_{1}^{\prime}\right) \cap \cdots \cap f_{n}^{-1}\left(C_{n}^{\prime}\right)$ is empty or

(i) the codimension of $Z$ in $X$ equals the sum

$$
\operatorname{codim}_{X}(Z)=\sum_{k=1}^{n} \operatorname{codim}_{\mathbb{R}^{r}}\left(C_{k}\right) ;
$$

(ii) $Z$ is pure-dimensional;

(iii) if a polyhedron $\alpha$ of $Z$ is contained in a polyhedron $\tau$ of $X$, the codimensions satisfy $\operatorname{codim}_{X}(\tau) \leq \operatorname{codim}_{Z}(\alpha)$ (in particular, the 
interior of a facet of $Z$ is contained in the interior of a facet of $X)$;

(iv) if the images $f_{k}(\alpha)$ of a polyhedron $\alpha$ of $Z$ are contained in polyhedra $\gamma_{k}$ of $C_{k}$, the codimensions satisfy $\sum_{k=1}^{n} \operatorname{codim}_{C_{k}}\left(\gamma_{k}\right) \leq$ $\operatorname{codim}_{Z}(\alpha)$.

Proof. It is easy to prove the statement in the case $n=1$ : (i), (ii) and (iii) are immediate consequences of Lemma 1.14 and (iv) follows from applying Lemma 1.14 to the $\left(r-\operatorname{codim}_{Z}(\alpha)-1\right)$-dimensional skeleton of $C_{1}$ (if $\gamma_{1}$ belonged to this skeleton, $\alpha$ would be contained in its preimage, which (for general translations) contradicts (i)). Now the statement follows if we apply the case of a single morphism to $f_{1} \times \cdots \times f_{n}: X \rightarrow\left(\mathbb{R}^{r}\right)^{n}$ and $C:=C_{1} \times \ldots \times C_{n}$.

Remark 1.16. Sticking to the notation of the previous statement, let us assume that $X$ is a cycle and that the maps $f_{k}$ are tropical morphisms. Moreover, we assume that the maps $f_{k}$ are projections (at least after composing with an isomorphism) and that the complexes $C_{k}$ are complete intersections. Then $f_{1}^{*}\left(C_{1}\right) \cdots f_{n}^{*}\left(C_{n}\right)$ is also a puredimensional complex of the same dimension as $f_{1}^{-1}\left(C_{1}^{\prime}\right) \cap \ldots \cap f_{n}^{-1}\left(C_{n}^{\prime}\right)$. Moreover, 1.12 shows that

$$
\left|f_{1}^{*}\left(C_{1}\right) \cdots f_{n}^{*}\left(C_{n}\right)\right| \subseteq f_{1}^{-1}\left(C_{1}^{\prime}\right) \cap \ldots \cap f_{n}^{-1}\left(C_{n}^{\prime}\right)
$$

holds. Hence, in this case we can think of $f_{1}^{*}\left(C_{1}\right) \cdots f_{n}^{*}\left(C_{n}\right)$ as being the polyhedral set $f_{1}^{-1}\left(C_{1}^{\prime}\right) \cap \cdots \cap f_{n}^{-1}\left(C_{n}^{\prime}\right)$ with the additional data of weights (some of which might be zero).

2. Intersections on the space of abstract curves. Let us start with a definition of smooth abstract curves. As a local model of a curve we will use the following fan. Let $e_{1}, \ldots, e_{r}$ be the standard basis in $\mathbb{R}^{r}$ and set $e_{0}:=-e_{1}-\cdots-e_{r}$. We define the one-dimensional fan

$$
L^{r}:=\left\{\{0\}, \mathbb{R}_{\geq}\left(-e_{0}\right), \ldots, \mathbb{R}_{\geq}\left(-e_{r}\right)\right\},
$$

with weights $\omega\left(\mathbb{R}_{\geq}\left(-e_{i}\right)\right)=1$ for all $i$. This fan is balanced because of $e_{0}+\cdots+e_{r}=0$. Note that this fan is also irreducible, as $e_{0}+\cdots+e_{r}=0$ is the only relation that the generating vectors fulfill. 
$\mathbb{R}$

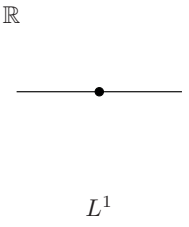

$\mathbb{R}^{2}$

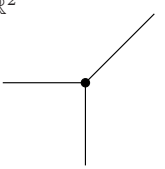

$L^{2}$

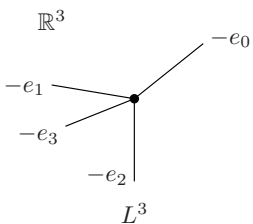

Definition 2.1. A smooth abstract curve $C$ is a one-dimensional connected cycle that is locally isomorphic to $L^{r}$ for suitable $r$, i.e., for each vertex $V$ in $C$ we have $\operatorname{Star}_{C}(V) \cong L^{\operatorname{val}(V)}$. The genus of $C$ is the first Betti number of $|C|$. An $n$-marked smooth abstract curve $\left(C, x_{1}, \ldots, x_{n}\right)$ is a smooth abstract curve $C$ with $n$ unbounded rays (called leaves), which are labeled by $x_{1}, \ldots x_{n}$. If we instead label the leaves by elements of some finite set $I$, we will call it an $I$-marked curve.

Remark 2.2. We will often omit the word "smooth" here as we will not consider other abstract curves (which are allowed to have different one-dimensional fans as local structures). Note that, by definition, $C$ is (locally) irreducible. We will always consider abstract curves up to isomorphisms.

Note that the valence of a vertex $V$ in $C$ completely fixes the local structure (which is $L^{\text {val }(V)}$ ). Hence, $C$ is in fact completely determined by the underlying metric graph, i.e., the combinatorial graph together with the (lattice length) of the edges. This is the definition in most existing literature, in particular in [8]. We will later be interested in parametrized curves, i.e., maps $f: C \rightarrow \mathbb{R}^{r}$. With the "metric graph" definition, the balancing condition has to be incorporated into the definition of these maps (see [8, subsection 4.1]). With our definition, we can just impose that $f$ should be a morphism of tropical cycles, giving the same result. Note that our definition also requires that a global embedding $C \subset \mathbb{R}^{N}$ of our curve exists (which we then forget as we identify isomorphic curves). This is done to avoid some technicalities involved in glueing abstract tropical cycles. However, we will see that (at least for rational curves) this is not a restriction as any "metric graph" curve can be embedded. 
Remark 2.3 (Smoothness criterion). Let us mention two simple criteria to decide whether a one-dimensional fan with $r+1$ rays is isomorphic to $L^{r}$ or not (i.e., smoothness criteria).

Let $X$ be a one-dimensional fan in $V=\Lambda \otimes \mathbb{R}$ with $r+1$ rays, all with weight 1 and generated by the primitive vectors $v_{0}, \ldots, v_{r}$. Let $V_{X}$ be the vector space spanned by $X$. Then the following are equivalent:

(i) $X$ is isomorphic to $L^{r}$.

(ii) The equations $v_{0}+\ldots+v_{r}=0, \operatorname{dim}\left(V_{X}\right)=r$ and $V_{X} \cap \Lambda=$ $\mathbb{Z} v_{0}+\cdots+\mathbb{Z} v_{r}$ hold

(iii) For arbitrary coefficients $\lambda_{0}, \ldots, \lambda_{r} \in \mathbb{R}$, we have

(a)

$$
\sum_{i=0}^{r} \lambda_{i} v_{i}=0 \Longleftrightarrow \lambda_{0}=\cdots=\lambda_{r} \Longleftrightarrow \lambda_{i}-\lambda_{j}=0 \quad \text { for all } i, j ;
$$

$$
\sum_{i=0}^{r} \lambda_{i} v_{i} \in \Lambda \Longleftrightarrow \lambda_{i}-\lambda_{j} \in \mathbb{Z} \quad \text { for all } i, j .
$$

The moduli space of (abstract smooth) n-marked rational tropical

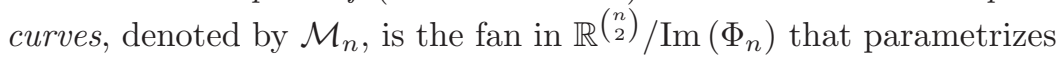
metric trees with positive lengths on the bounded edges (and infinite lengths on the unbounded edges). The explicit construction of this space can be found in $[\mathbf{2 0}, \mathbf{2 1}]$ and $\left[\mathbf{8}\right.$, Section 3]. The cones of $\mathcal{M}_{n}$ are in one-to-one correspondence with combinatorial types of $n$-marked trees (with 2-valent vertices removed), and the dimension of a cone equals the number of bounded edges in the respective combinatorial type. A general point in $\mathcal{M}_{n}$ (i.e., an element in the interior of a facet) is a three-valent metric tree with $n-3$ bounded edges (hence, $\left.\operatorname{dim}\left(\mathcal{M}_{n}\right)=n-3\right)$. When all facets are equipped with weight $1, \mathcal{M}_{n}$ fulfills the balancing condition. Hence, $\mathcal{M}_{n}$ is a tropical fan cycle. We denote the leaves by $x_{1}, \ldots, x_{n}$. If we work with $\mathcal{M}_{n+1}$, the extra leaf is labeled by $x_{0}$. As $\mathcal{M}_{3}$ is just a single point, we assume $n \geq 4$ in most cases. 


$$
V_{I \mid J}:=\quad \begin{gathered}
x_{i}, \\
i \in I
\end{gathered} \geqslant \underset{\substack{\text { edge of length } 1 \\
j \in J}}{x_{j},} \in \mathcal{M}_{n}
$$

The notation $I \mid J$ denotes a non-trivial partition of $[n]=\{1, \ldots, n\}$ (or of $\{0\} \cup[n]$ if we work with $\mathcal{M}_{n+1}$ ) into the two disjoint subsets $I$ and $J$. In most cases-the few exceptions will be mentioned-we will consider this partition to be unordered. Occasionally, we use $I^{c}$ to denote the complement of $I$ and write $I \mid I^{c}$. If $|I| \neq 1 \neq|J|$, such a partition describes a ray in $\mathcal{M}_{n}$ generated by the metric tree $V_{I \mid J} \in \mathcal{M}_{n}$ with only one bounded edge: An edge of a tree is uniquely determined by the partition $I \mid J$ obtained when removing the edge. In this sense, we can regard the partitions $I \mid J$ as "global" labels of the edges of a tree, where $I \mid J$ labels the leaf $x_{i}$ if $I=\{i\}$ or $J=\{i\}$, and a bounded edge otherwise. A cone $\tau$ of $\mathcal{M}_{n}$ is generated by the vectors $V_{I \mid J}$ for all partitions which correspond to edges in the combinatorial type of $\tau$. In particular, it is natural to use the lengths of the bounded edges as local coordinates of a cone of $\mathcal{M}_{n}$ - this identifies each cone $\tau$ of $\mathcal{M}_{n}$ with the positive orthant of $\mathbb{R}^{\operatorname{dim}(\tau)}$.

Let us make some remarks here. We sometimes also think of $V_{I \mid J}$

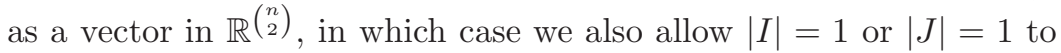
get simpler formulas. However, as

$$
V_{\{k\} \mid[n] \backslash\{k\}}=\Phi_{n}(0, \ldots, 0,1,0, \ldots, 0),
$$

these vectors vanish modulo $\operatorname{Im}\left(\Phi_{n}\right)$.

Note that, for the following purposes, the underlying lattice of

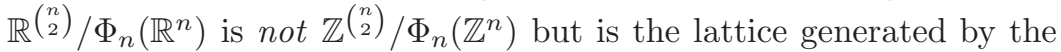
vectors $V_{I \mid J}$, denoted by $\Lambda_{n}$ (see [8, subsection 3.3]). This is a technical issue, as it does not change the lattices of the cones $\Lambda_{\tau}$, $\tau \in \mathcal{M}_{n}$ but is necessary for making maps such as forgetful maps integer affine.

As mentioned above, any metric tree can be realized uniquely by a smooth rational curve in the sense of Definition 2.1 (we actually prove this in Proposition 2.19). Therefore, $\mathcal{M}_{n}$ really parametrizes what is promised by its name. 
Comparing $\mathcal{M}_{n}$ to its classical counterpart, note that we will stick to the non-compact part of smooth curves and will not use a compactification. However, the "recursive structure" of the boundary known from the classical moduli space of stable curves is already visible in $\mathcal{M}_{n}$ (without adding a "boundary"). Namely, let $\tau$ be a cone of $\mathcal{M}_{n}$, and let $\Gamma$ denote the corresponding combinatorial type of $n$-marked trees. Then it is easy to check that the star around $\tau$ satisfies

$$
\operatorname{Star}_{\mathcal{M}_{n}}(\tau)=\prod_{\substack{\nu \text { vertex } \\ \text { of } \Gamma}} \mathcal{M}_{\operatorname{val}(\nu)},
$$

i.e., can be described as the product of "smaller" moduli spaces.

We will now define divisors, respectively, rational functions that play the role of "boundary" divisors in our moduli space. More precisely, if we actually would compactify $\mathcal{M}_{n}$, these divisors should be rationally equivalent to the actual boundary divisors. All these divisors lie in the codimension 1 skeleton of $\mathcal{M}_{n}$, and they therefore represent higher-valent curves. As $\mathcal{M}_{n}$ is simplicial, we can define a rational function on $\mathcal{M}_{n}$ by assigning an integer to each $I \mid J$ : the integers are the values of the function at $V_{I \mid J}$, and on each cone we extend the function by linearity.

Definition 2.4. We define the rational function $\varphi_{I \mid J}$ by

$$
\varphi_{I \mid J}\left(V_{I^{\prime} \mid J^{\prime}}\right):= \begin{cases}1 & \text { if } I=I^{\prime} \text { or } I=J^{\prime} \\ 0 & \text { otherwise. }\end{cases}
$$

Furthermore, we use the notation,

$$
\varphi_{k, l}:=\varphi_{\{k, l\} \mid[n] \backslash\{k, l\}},
$$

for $k \neq l$.

The ridges (codimension 1 cells) of $\mathcal{M}_{n}$ correspond to combinatorial types of curves with one 4 -valent vertex, which we will draw like this:

$$
\stackrel{A}{D} \times \stackrel{B}{C} .
$$

Here $A, B, C$ and $D$ denote the four parts of the combinatorial type adjacent to the 4 -valent vertex and, by abuse of notation, also the 


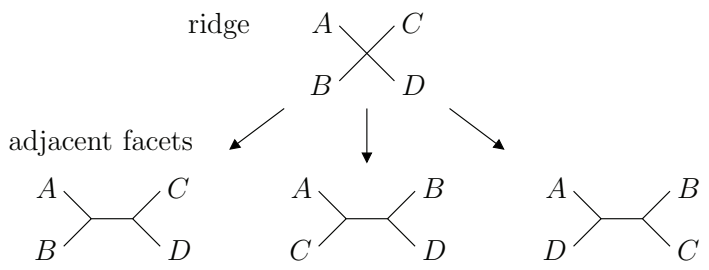

sets of leaves belonging to this part (as, in most cases, this is the only information needed). If we want to compute the weight of a ridge ${ }_{D}^{A} \times{ }_{C}^{B}$ in the divisor of a rational function on $\mathcal{M}_{n}$, we need to know how $\mathcal{M}_{n}$ looks locally around ${ }_{D}^{A} \times{ }_{C}^{B}$. Obviously, $\operatorname{Star}_{\mathcal{M}_{n}}\left({ }_{D}^{A} \times{ }_{C}^{B}\right)$ contains three facets corresponding to the three possibilities of "resolving" the 4valent vertex by inserting a new bounded edge. The (representatives of the) primitive vectors are $V_{A \cup B \mid C \cup D}, V_{A \cup C \mid B \cup D}$ and $V_{A \cup D \mid B \cup C}$. For the balancing condition around ${ }_{D}^{A} \times{ }_{C}^{B}$, it suffices to show the equation:

$$
\begin{aligned}
V_{A \cup B \mid C \cup D}+ & V_{A \cup C \mid B \cup D}+V_{A \cup D \mid B \cup C} \\
& =V_{A \mid B \cup C \cup D}+V_{B \mid A \cup C \cup D}+V_{C \mid A \cup B \cup D}+V_{D \mid A \cup B \cup C},
\end{aligned}
$$

as all vectors on the right hand side lie in the vector space spanned by the ridge ${ }_{D}^{A} \times{ }_{C}^{B}$, as required. But the equation follows from the fact that, on the level of metric trees, the distance between two marked leaves is identical on both sides. If both leaves belong to the same set $A, B, C, D$, the distance is 0 ; if not, it is 2 .

Let us now compute the divisors of the functions $\varphi_{I \mid J}$. In the following, a formula involving $I, J$ and $A, B, C, D$ stands for all permuted formulas as well, e.g., $I=A$ means " $I=A$ or $I=B$ or $J=A \ldots$.

Lemma 2.5. The boundary divisor $\operatorname{div}\left(\varphi_{I \mid J}\right)$ carries the weight function

$$
\omega_{\varphi_{I \mid J}}\left({ }_{D}^{A} \times{ }_{C}^{B}\right)= \begin{cases}1 & \text { if } I=A \cup B, \\ -1 & \text { if } I=A, \\ 0 & \text { otherwise. }\end{cases}
$$

Proof. Following from the previous discussion, the weight of ${ }_{D}^{A} \times{ }_{C}^{B}$ in $\operatorname{div}\left(\varphi_{I \mid J}\right)$ is, by definition, 


$$
\begin{aligned}
\omega_{\varphi_{I \mid J}}\left({ }_{D}^{A} \times{ }_{C}^{B}\right)= & \varphi_{I \mid J}\left(V_{A \cup B \mid C \cup D}\right)+\varphi_{I \mid J}\left(V_{A \cup C \mid B \cup D}\right) \\
& +\varphi_{I \mid J}\left(V_{A \cup D \mid B \cup C}\right)-\varphi_{I \mid J}\left(V_{A \mid B \cup C \cup D}\right) \\
& -\varphi_{I \mid J}\left(V_{B \mid A \cup C \cup D}\right)-\varphi_{I \mid J}\left(V_{C \mid A \cup B \cup D}\right) \\
& -\varphi_{I \mid J}\left(V_{D \mid A \cup B \cup C}\right) .
\end{aligned}
$$

Hence, this weight is 1 if $I$ is the union of two of the sets $A, B, C, D$ and -1 if $I$ equals one of the four sets. Otherwise, it is 0 .

These divisors were computed before by Herold (see [11]).

Remark 2.6. In terms of the general toric geometry rules, the functions $\varphi_{I \mid J}$, respectively the $\operatorname{divisors} \operatorname{div}\left(\varphi_{I \mid J}\right)$, are the tropical analogues of the irreducible components of the boundary of the classical moduli space of stable curves $\bar{M}_{0, n}$. Instead of using this fact explicitly, in the following we will show in purely tropical terms that the tropical divisors show the same intersection-theoretic behavior as their classical counterparts. (We will need this anyway when dealing with parametrized curves later on.)

Lemma 2.7. The equation,

$$
\varphi_{i, j} \cdot \varphi_{i, k} \cdot \mathcal{M}_{n}=0
$$

holds for $n \geq 4$ and pairwise different $i, j, k \in[n]$.

Proof. An abstract curve $C$ cannot simultaneously have bounded edges with partitions $\{i, j\} \mid\{i, j\}^{c}$ and $\{i, k\} \mid\{i, k\}^{c}$ (as, for example, the first partition forces $i$ and $k$ to be adjacent to the same threevalent vertex). Let $C$ be a curve in $\left|\varphi_{i, k}\right|$. At least after resolving a four-valent vertex, it contains an edge with partition $\{i, k\} \mid\{i, k\}^{c}$ and can therefore not contain an edge with partition $\{i, j\} \mid\{i, j\}^{c}$. But $\varphi_{i, j}$ just measures the length of such an edge, if present. Thus, $\varphi_{i, j}\left|\varphi_{i, k}\right| \equiv 0$.

Analogues of $\Psi$-classes on tropical $\mathcal{M}_{n}$ have been defined by Mikhalkin ([20]). Their intersections were studied in in [15]. We use the notion $\Psi$-divisor, instead of $\Psi$-class, to emphasize that, in contrast 
to the algebro-geometric case, tropically $\Psi$-divisors are not defined up to rational equivalence. (Again, in the toric geometry language, the tropical $\Psi$-divisors are just the Minkowski weights associated to the classical ones.) In order to perform intersections, we need to describe our $\Psi$-divisors by rational functions. Let us recall the important definitions and results of [15] here.

Definition 2.8. We define the $k$ th $\Psi$-function $\psi_{k}$ by

$$
\psi_{k}\left(V_{I \mid J}\right):=\frac{|I|(|I|-1)}{(n-1)(n-2)},
$$

for all partitions $I \mid J$ with $|I|,|J| \geq 2$ and $k \in J$.

Remark 2.9. Our function $\psi_{k}$ equals the function $1 /\left(\begin{array}{c}n-1 \\ 2\end{array}\right) f_{k}$ defined in [15] (which follows from [15, Lemma 2.6]). In particular, $\psi_{k}$ is a convex function (cf., [15, Remark 2.5]). Note that, in this paper, $\psi_{k}$ and $\varphi_{I \mid J}$ denote functions and not their corresponding divisors. On the other hand, as mentioned in subsection 1.6, this is only a matter of notation. For intersection-theoretic purposes, the actual choice of a function defining the same divisor does not matter.

Remark 2.10. Obviously, the numbers $\psi_{k}\left(V_{I \mid J}\right)$ are only rational. A generalization of intersection theory to rational numbers is straightforward but also essentially unnecessary. The weights of the divisor of $\psi_{k}$ turn out to be integers (see the following proposition) and there exist integer rational functions producing the same divisor (see Lemma 2.24). This particular function $\psi_{k}$ was chosen in [15] because of its symmetry.

Proposition 2.11. (See [15, subsection 3.5]). The divisor $\operatorname{div}\left(\psi_{k}\right)$ consists of the cones corresponding to trees where the marked leaf $k$ is at a four-valent vertex, i.e., the weight of a facet in $\operatorname{div}\left(\psi_{k}\right)$ (which is a ridge in $\mathcal{M}_{n}$ ) is:

$$
\omega_{\psi_{k}}\left({ }_{D}^{A} \times{ }_{C}^{B}\right)= \begin{cases}1 & \text { if }\{k\}=A \\ 0 & \text { otherwise. }\end{cases}
$$


Remark 2.12. As mentioned above, in toric geometry language and taking into consideration the embedding of the classical moduli space of stable curves in the toric variety associated to $\mathcal{M}_{n}$, it is easy to see that $\operatorname{div}\left(\psi_{k}\right)$ indeed represents the Minkowski weight associated to the classical $k$ th $\Psi$-class. To check this, consider the one-dimensional boundary stratum $S$ in the classical moduli space corresponding to reducible curves with dual graph ${ }_{D}^{A} \times{ }_{C}^{B}$. Each of such curves contains exactly one $\mathbb{P}^{1}$-component with four special points, whereas all other components carry exactly three special points and therefore are rigid. Hence, $S$ is isomorphic to the moduli space of four-marked stable curves $\bar{M}_{0,4} \cong \mathbb{P}^{1}$. Let $\mathcal{L}_{k}$ be the line bundle whose fibre over a point corresponding to a curve $C$ is the cotangent space $T_{x_{k}}^{*} C$ at the marked point $x_{k}$. By definition, the classical $\Psi$-class is just the first Chern class of this line bundle. To compute the associated Minkowski weight, we should evaluate this Chern class on the one-dimensional boundary stratum described above. If $A=\{k\}$, this means that $x_{k}$ is one of the four special points on the non-rigid component. It follows that $\left.\mathcal{L}_{k}\right|_{S}$ is equal to the corresponding $\Psi$ line bundle on $\bar{M}_{0,4}$ and we can compute $\operatorname{deg}\left(\left.\mathcal{L}_{k}\right|_{S}\right)=1$. (For example, representing $\bar{M}_{0,4}$ as the pencil of conics through four points in the plane, there is exactly one conic with prescribed tangent line at one of the four points.) If $A \neq\{k\}$, the marked point $x_{k}$ lies on one of the rigid components. Therefore, $\left.\mathcal{L}_{k}\right|_{S}$ is the trivial line bundle and $\operatorname{deg}\left(\left.\mathcal{L}_{k}\right|_{S}\right)=0$. This reproduces the weights from our proposition. Again, let us emphasize that we do not really use this derivation as our arguments are purely tropical.

Notation 2.13. As in the conventional case we will introduce the following $\tau$-notation that makes formulas shorter and hides "unimportant" data such as the number of marked leaves. For any positive integers $a_{1}, \ldots, a_{n}$, we define

$$
\left(\tau_{a_{1}} \cdot \ldots \cdot \tau_{a_{n}}\right):=\psi_{1}^{a_{1}} \cdot \ldots \cdot \psi_{n}^{a_{n}} \cdot \mathcal{M}_{n} .
$$

Every factor $\tau_{a_{k}}$ stands for a marked leaf, and the index $a_{k}$ serves as the exponent with which the corresponding $\Psi$-function appears in the intersection product. If $\sum a_{k}=\operatorname{dim}\left(\mathcal{M}_{n}\right)=n-3$, the above cycle is zero-dimensional (in fact, its only point corresponds to the curve without bounded edges where all leaves are adjacent to one single 
vertex), and we define

$$
\left\langle\tau_{a_{1}} \cdot \ldots \cdot \tau_{a_{n}}\right\rangle:=\operatorname{deg}\left(\psi_{1}^{a_{1}} \cdot \ldots \cdot \psi_{n}^{a_{n}} \cdot \mathcal{M}_{n}\right) .
$$

The main theorem of [15] computes these intersection products of $\Psi$-divisors.

Theorem 2.14. (Intersections of $\Psi$-divisors for abstract curves, see [15, subsection 4.1]). The intersection product $\left(\tau_{a_{1}} \cdot \ldots \cdot \tau_{a_{n}}\right)$ is the subfan of $\mathcal{M}_{n}$ consisting of the closure of the cones of dimension $n-3-\sum_{i=1}^{n} a_{i}$ whose interior curves $C$ have the following property.

Let $k_{1}, \ldots, k_{q} \subseteq N$ be the marked leaves adjacent to a vertex $V$ of $C$. Then the valence of $V$ is

$$
\operatorname{val}(V)=a_{k_{1}}+\ldots+a_{k_{q}}+3
$$

Let us define the multiplicity of this vertex to be $\operatorname{mult}(V):=\left(\begin{array}{c}\operatorname{val}(V)-3 \\ a_{k_{1}}, \ldots, a_{k_{q}}\end{array}\right)$. Then the weight of such a cone $\sigma$ in $X$ is

$$
\omega_{X}(\sigma)=\prod_{V} \operatorname{mult}(V),
$$

where the product runs through all vertices $V$ of an interior curve of $\sigma$.

In this section we reprove the zero-dimensional case of this theorem (see Remark 2.22). To do this, we first have to analyze how $\Psi$ and boundary divisors intersect and how they behave when pulled back or pushed forward along forgetful morphisms.

Lemma 2.15. The following holds

$$
\varphi_{i, j} \cdot \psi_{i} \cdot \mathcal{M}_{n}=0
$$

for $n \geq 4$ and $i \neq j \in[n]$.

Proof. Curves in $\left|\psi_{i}\right|$ cannot contain a bounded edge with partition $\{i, j\} \mid\{i, j\}^{c}$, as the leaf $i$ does not lie at a three-valent vertex. Thus, $\varphi_{i, j}$ vanishes on $\left|\psi_{i}\right|$. 
The forgetful map $\mathcal{M}_{n+1} \rightarrow \mathcal{M}_{n}$ that forgets the extra leaf $x_{0}$ is denoted by $\mathrm{ft}_{0}$ (cf., [9, subsection 4.1] and [8, subsection 3.8]). By [8, subsection 3.9], this map is a tropical morphism. Therefore, we can ask how $\Psi$-functions behave when pulled back along $\mathrm{ft}_{0}$.

Lemma 2.16 (Pull-back of $\Psi$-functions). Let $n \geq 4$, and let $\mathrm{ft}_{0}$ : $\mathcal{M}_{n+1} \rightarrow \mathcal{M}_{n}$ be the morphism that forgets the leaf $x_{0}$. For $k \in[n]$, the following holds:

$$
\operatorname{div}\left(\psi_{k}\right)=\operatorname{div}\left(\mathrm{ft}_{0}^{*} \psi_{k}\right)+\operatorname{div}\left(\varphi_{0, k}\right) .
$$

Proof. This can be proven by explicitly computing the weights of the codimension 1 faces of the three divisors. We distinguish four cases (up to renaming $A, B, C$ and $D$ ):

\begin{tabular}{c|ccc}
$\omega_{f}\left({ }_{D}^{A} \times{ }_{C}^{B}\right)$ & $f=\psi_{k}$ & $f=\mathrm{ft}_{0}^{*} \psi_{k}$ & $f=\varphi_{0, k}$ \\
\hline$A=\{0, k\}$ & 0 & 1 & -1 \\
$A=\{0\}, B=\{k\}$ & 1 & 0 & 1 \\
$A=\{0, \ldots\}, B=\{k\}$ & 1 & 1 & 0 \\
otherwise & 0 & 0 & 0
\end{tabular}

Corollary 2.17. Let $n \geq 4$, and let $\mathrm{ft}_{0}: \mathcal{M}_{n+1} \rightarrow \mathcal{M}_{n}$ be the morphism that forgets the leaf $x_{0}$. Then, for $k \in[n]$, the following formulas hold:

(i) $\varphi_{0, k}^{2}=\mathrm{ft}_{0}^{*}\left(\psi_{k}\right) \cdot \varphi_{0, k}$,

(ii) $\psi_{k}^{a}=\mathrm{ft}_{0}^{*}\left(\psi_{k}\right)^{a}+\mathrm{ft}_{0}^{*}\left(\psi_{k}\right)^{a-1} \cdot \varphi_{0, k}$,

(iii) $\psi_{k}^{a}=\mathrm{ft}_{0}^{*}\left(\psi_{k}\right)^{a}+(-1)^{a-1} \varphi_{0, k}^{a}$.

Proof. All the formulas follow directly from Lemmas 2.15 and 2.16 .

Lemma 2.18. Let $n \geq 4$, let $\mathrm{ft}_{0}: \mathcal{M}_{n+1} \rightarrow \mathcal{M}_{n}$ be the morphism that forgets the leaf $x_{0}$ and choose $k \in[n]$. Then

$$
\mathrm{ft}_{0 *}\left(\operatorname{div}\left(\varphi_{0, k}\right)\right)=\mathrm{ft}_{0 *}\left(\operatorname{div}\left(\psi_{k}\right)\right)=\mathcal{M}_{n} .
$$


Proof. We show $\mathrm{ft}_{0 *}\left(\operatorname{div}\left(\varphi_{0, k}\right)\right)=\mathcal{M}_{n}$ by direct computation. Let $\sigma^{\prime}$ be a facet of $\mathcal{M}_{n}$ corresponding to a three-valent combinatorial type. Let $V$ be the vertex adjacent to $k$. Then there exists precisely one cone $\sigma$ in $\operatorname{div}\left(\varphi_{0, k}\right)$ whose image under $\mathrm{ft}_{0}$ is $\sigma^{\prime}$, namely, the cone obtained by attaching the additional leaf $x_{0}$ to the vertex $V$. Moreover, on such a cone, the length of the bounded edges remain unchanged under $\mathrm{ft}_{0}$, and therefore $\mathrm{ft}_{0}\left(\Lambda_{\sigma}\right)=\Lambda_{\sigma^{\prime}}$. On the other hand, cones in $\operatorname{div}\left(\varphi_{0, k}\right)$ with negative weight are not mapped injectively, as in this case $x_{0}$ is adjacent to a three-valent vertex, and stabilization is needed. This shows that $\mathrm{ft}_{0 *}\left(\operatorname{div}\left(\varphi_{0, k}\right)\right)=\mathcal{M}_{n}$.

The equation $\mathrm{ft}_{0 *}\left(\operatorname{div}\left(\psi_{k}\right)\right)=\mathcal{M}_{n}$ follows from the same argument or by using Lemma 2.16, the projection formula and $\mathrm{ft}_{0 *}\left(\mathcal{M}_{n+1}\right)=0$ (because the dimension is too big).

It is well known that, for the classical moduli space $\bar{M}_{0, n}$, the forgetful morphism plays the role of the universal family (cf., [16, subsection 1.3]). In the tropical setting, we can prove the following statement.

Proposition 2.19 (Family property of $\mathrm{ft}_{0}$ for abstract curves). Let $p$ be a point in $\mathcal{M}_{n}$, and let $C_{p}=\mathrm{ft}_{0}^{-1}(p)$ be the fibre of $p$ under the forgetful morphism $\mathrm{ft}_{0}: \mathcal{M}_{n+1} \rightarrow \mathcal{M}_{n}$. Then the following holds:

(i) $C_{p}$ has the canonical structure of a one-dimensional polyhedral complex.

(ii) The leaves of $C_{p}$ (as the graph itself) are the facets where $x_{0}$ and another leaf $x_{i}$ lie at the same 3-valent vertex (i.e., the leaves are given by $\left.L_{i}:=\overline{\left\{y \in C_{p} \mid \varphi_{0, i}(y)>0\right\}}\right)$. Moreover, $p \in \mathcal{M}_{n}$ represents the $n$-marked metric graph $\left(C_{p}, L_{1}, \ldots, L_{n}\right)$.

(iii) When we equip all its facets with weight $1, C_{p}$ is a smooth abstract curve (in the sense of Definition 2.1).

(iv) Let $\sum_{k} \mu_{k} p_{k}=\varphi_{1} \cdot \ldots \cdot \varphi_{n-3} \cdot \mathcal{M}_{n}$ be a zero-dimensional cycle in $\mathcal{M}_{n}$ obtained as the intersection product of convex functions $\varphi_{j}$. Then

$$
\mathrm{ft}_{0}^{*}\left(\varphi_{1}\right) \cdot \ldots \cdot \mathrm{ft}_{0}^{*}\left(\varphi_{n-3}\right) \cdot \mathcal{M}_{n+1}=\sum_{k} \mu_{k} C_{p_{k}} .
$$

We write this as $\mathrm{ft}_{0}^{*}\left(\sum_{k} \mu_{k} p_{k}\right)=\sum_{k} \mu_{k} C_{p_{k}}$. 


\section{Proof.}

(i) As a polyhedral complex, $C_{p}$ consists of the polyhedra $\left.\mathrm{ft}_{0}\right|_{\sigma} ^{-1}(p)$ for each cone $\sigma$ of $\mathcal{M}_{n+1}$. The dimension of these polyhedra can be at most one as $\operatorname{dim}\left(f_{0}(\sigma)\right) \geq \operatorname{dim}(\sigma)-1$ (it depends on whether $x_{0}$ is adjacent to a three-valent or higher-valent vertex).

(ii) Let $\Gamma_{p}$ denote the $n$-marked metric graph represented by $p$. The bijective map $\Gamma_{p} \rightarrow C_{p}$ indicated in the picture identifies the two graphs.

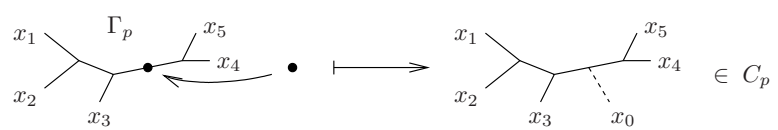

(iii) Let $V$ be a vertex of $C_{p}$. It corresponds to the metric graph $\Gamma_{p}$ with the extra leaf $x_{0}$ adjacent to one of the vertices. Let us label the other edges adjacent to this vertex by $1, \ldots, m$, and let us divide the other leaves $[n]=I_{1} \cup \ldots \cup I_{m}$ according to via which edge one reaches $x_{i}$ from $x_{0}$. There are $m$ facets in $C_{p}$ adjacent to $V$ corresponding to moving $x_{0}$ on one of the edges. Hereby, one has to shorten the edge $I_{k} \mid I_{k}^{c}$ as much as the length of $I_{k} \cup\left\{x_{0}\right\} \mid\left(I_{k} \cup\left\{x_{0}\right\}\right)^{c}$ increases.

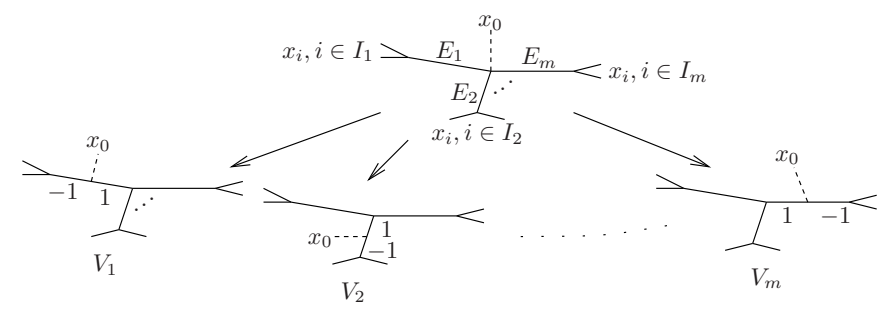

Thus, the primitive integer vector of the corresponding facet with respect to $V$ is given by

$$
V_{k}:=V_{I_{k} \cup\left\{x_{0}\right\}}-V_{I_{k}} .
$$

Note that this formula as well as the following ones also hold in the case that $I_{k}$ consists only of a single leaf $x_{i}$ (which means $x_{i}$ is adjacent to the same vertex as $\left.x_{0}\right)$, as $V_{\left\{x_{i}\right\}}=$

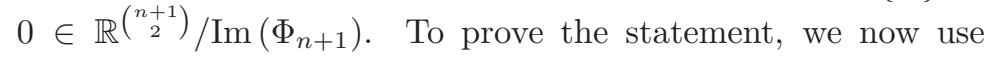
Remark 2.3 and verify conditions (i) and (ii), which can be done 
by applying some formulas of [15]. Let $\mathcal{S}$ be the set of twoelement subsets of $[n]$ (i.e., not containing 0 ). It follows from $[\mathbf{1 5}$, subsections 2.3, 2.4, 2.6] that the vectors $V_{S}, S \in \mathcal{S}$ fulfill (i) and

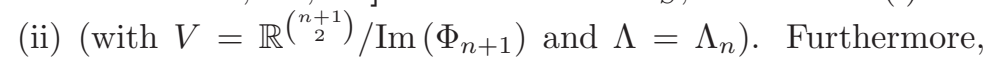
[15, subsection 2.6] gives us a representation of our vectors in terms of the vectors $V_{S}$, namely,

$$
\begin{gathered}
V_{I_{k}}=\sum_{\substack{S \in \mathcal{S} \\
S \subseteq I_{k}}} V_{S} \\
V_{I_{k} \cup\left\{x_{0}\right\}}=\sum_{\substack{S \in \mathcal{S} \\
S \cap I_{k}=\emptyset}} V_{S}=-\left(\sum_{\substack{S \in \mathcal{S} \\
S \cap I_{k} \neq \emptyset}} V_{S}\right),
\end{gathered}
$$

and therefore,

$$
V_{k}=-\left(\sum_{S \in \mathcal{S}}\left|S \cap I_{k}\right| \cdot V_{S}\right) .
$$

Now let $\lambda_{1}, \ldots, \lambda_{m}$ be arbitrary real coefficients. Then we obtain the formula

$$
\sum_{k=1}^{m} \lambda_{k} V_{k}=-\left(\sum_{\substack{\{i, j\} \in \mathcal{S} \\ i \in I_{k}, j \in I_{k^{\prime}}}}\left(\lambda_{k}+\lambda_{k^{\prime}}\right) \cdot V_{\{i, j\}}\right) .
$$

Now all differences of two coefficients on the left hand side $\lambda_{k}-\lambda_{k}^{\prime}$ can be obtained as differences of two coefficients on the right hand side (choose elements $i \in I_{k}, j \in I_{k^{\prime}}, l \in I_{k^{\prime \prime}}$; then the coefficients of $V_{\{i, l\}}$ and $V_{\{j, l\}}$ differ by $\left.\lambda_{k}+\lambda_{k^{\prime \prime}}-\lambda_{k^{\prime}}-\lambda_{k^{\prime \prime}}=\lambda_{k}-\lambda_{k^{\prime}}\right)$. Conversely, a right hand side difference of coefficients equals the sum of two left hand side differences. (The coefficients of $V_{\left\{i_{1}, i_{2}\right\}}$ and $V_{\left\{j_{1}, j_{2}\right\}}$ differ by $\left(\lambda_{k_{1}}-\lambda_{l_{1}}\right)+\left(\lambda_{k_{2}}-\lambda_{l_{2}}\right)$, where $i_{1} \in I_{k_{1}}$, $i_{2} \in I_{k_{2}}, j_{1} \in I_{l_{1}}, j_{2} \in I_{l_{2}}$.) Hence, as Remark 2.3 conditions (i) and (ii) hold for the vectors $V_{S}$, they also hold for the vectors $V_{k}$. 
(iv) First of all, the set-theoretic equation

$$
\begin{aligned}
\mid \mathrm{ft}_{0}^{*}\left(\varphi_{1}\right) \cdot \ldots \cdot \mathrm{ft}_{0}^{*}\left(\varphi_{n-3}\right) & \cdot \mathcal{M}_{n+1} \mid \\
& \subseteq \mathrm{ft}_{0}^{-1}\left(\left|\varphi_{1} \cdot \ldots \cdot \varphi_{n-3} \cdot \mathcal{M}_{n}\right|\right)=\bigcup_{k}\left|C_{p_{k}}\right|
\end{aligned}
$$

follows from Proposition 1.11. But the sets $\left|C_{p_{k}}\right|$ are pairwise disjoint (as they are fibres of pairwise different points) and belong to irreducible cycles (as the curves $C_{p_{k}}$ are smooth abstract curves). Thus, any one-dimensional cycle whose support lies in $\bigcup_{i}\left|C_{p_{k}}\right|$ is actually a sum $\sum_{k} \lambda_{k} C_{p_{k}}, \lambda_{k} \in \mathbb{Z}$.

So it remains to check that, in our case, these coefficients $\lambda_{k}$ coincide with $\mu_{k}$. To do this, we choose an arbitrary leaf $x_{i} \neq x_{0}$ and consider the function $\varphi_{0, i}$ on $C_{p_{k}}$. On the leaf $L_{i}$ of $C_{p_{k}}$, where $x_{0}$ and $x_{i}$ are adjacent to the same three-valent vertex, it measures the length of the third edge, elsewhere it is constantly zero. Thus, $\varphi_{0, i} \cdot C_{p_{k}}=V_{p_{k}}$, where $V_{p_{k}}$ is the vertex of $C_{p_{k}}$ adjacent to $L_{i}$ (where $x_{0}$ and $x_{i}$ lie together at a higher-valent vertex). Thus, we get

$$
\mathrm{ft}_{0 *}\left(\varphi_{0, i} \cdot\left(\sum_{k} \lambda_{k} C_{p_{k}}\right)\right)=\mathrm{ft}_{0 *}\left(\sum_{k} \lambda_{k} V_{p_{k}}\right)=\sum_{k} \lambda_{k} p_{k} .
$$

On the other hand, we can use the projection formula and Lemma 2.18 and compute

$$
\begin{aligned}
\mathrm{ft}_{0 *}\left(\varphi_{0, i} \cdot \mathrm{ft}_{0}^{*}\left(\varphi_{1}\right)\right. & \left.\cdot \ldots \cdot \mathrm{ft}_{0}^{*}\left(\varphi_{n-3}\right) \cdot \mathcal{M}_{n+1}\right) \\
& =\varphi_{1} \cdot \ldots \cdot \varphi_{n-3} \cdot \mathrm{ft}_{0 *}\left(\varphi_{0, i} \cdot \mathcal{M}_{n+1}\right)=\sum_{k} \mu_{k} p_{k} .
\end{aligned}
$$

Comparing the coefficients proves the statement.

Remark 2.20. Hence, there is a one-to-one correspondence between curves according to the "old" definition (i.e., as metric graphs) and Definition 2.1. In particular, $\mathcal{M}_{n}$ parametrizes smooth abstract curves in our sense. 
Theorem 2.21 (String equation for abstract curves). For zerodimensional intersection products of $\Psi$-divisors the following holds.

$$
\left\langle\tau_{0} \prod_{k=1}^{n} \tau_{a_{k}}\right\rangle_{d}=\sum_{i=1}^{n}\left\langle\tau_{a_{i}-1} \prod_{k \neq i} \tau_{a_{k}}\right\rangle_{d}
$$

Proof. The proof is identical to the algebro-geometric one. We must compute the degree of the intersection product $\prod_{k=1}^{n} \psi_{k}^{a_{k}} \cdot \mathcal{M}_{n+1}$. First we replace each term $\psi_{k}^{a_{k}}(k \neq 0)$ by ft $\mathrm{ft}_{0}^{*}\left(\psi_{k}\right)^{a_{k}}+\mathrm{ft}_{0}^{*}\left(\psi_{k}\right)^{a_{k}-1} \cdot \varphi_{0, k}$ using Corollary 2.17 (ii) and multiplying the product out. As $\varphi_{0, k} \cdot \varphi_{0, k^{\prime}}=0$ for $k \neq k^{\prime}$ (see equation (2.7)), we only get the following $n+1$ terms.

$$
\prod_{k=1}^{n} \mathrm{ft}_{0}^{*}\left(\psi_{k}\right)^{a_{k}} \cdot \mathcal{M}_{n+1}+\sum_{i=1}^{n} \mathrm{ft}_{0}^{*}\left(\psi_{i}\right)^{a_{i}-1} \cdot \prod_{k \neq i} \mathrm{ft}_{0}^{*}\left(\psi_{k}\right)^{a_{k}} \cdot \varphi_{0, i} \cdot \mathcal{M}_{n+1}
$$

Now we push this cycle forward along $\mathrm{ft}_{0}$ and use projection formula. The first term vanishes for dimension reasons and, as $\varphi_{0, i}$ pushes forward to $\mathcal{M}_{n}$ by Lemma 2.18, the other terms provide the desired result.

Remark 2.22. As in the classical case, the string equation suffices to compute all intersection numbers of $\Psi$-divisors of abstract curves. Namely, if $\sum a_{i}=n-3$, the equation

$$
\left\langle\tau_{a_{1}} \cdot \ldots \cdot \tau_{a_{n}}\right\rangle=\frac{(n-3) !}{a_{1} ! \cdot \ldots \cdot a_{n} !}
$$

holds. This was proven in [15, subsection 4.2] using the paper's main theorem [15, subsection 4.1] (cited here in Theorem 2.14). Note, however, that in order to prove the string equation it was not necessary to use [15, subsection 4.1]. Another independent proof of the above equality is given in [13, Proposition 7.4].

Lemma 2.23. Let $n>4$, and let $\mathrm{ft}_{0}: \mathcal{M}_{n+1} \rightarrow \mathcal{M}_{n}$ be the morphism that forgets the last leaf. Then

$$
\mathrm{ft}_{0 *}\left(\operatorname{div}\left(\varphi_{I \mid J}\right)\right)= \begin{cases}\mathcal{M}_{n} & \text { if } I=\{0, k\} \text { or } J=\{0, k\} \text { for some } k \in[n] \\ 0 & \text { otherwise. }\end{cases}
$$


Proof. The first part is shown in Lemma 2.18. So let us prove the second part. First, we choose $i \in I$ and $j \in J$, both different from 0 . Consider a facet $\sigma^{\prime}$ in $\mathcal{M}_{n}$ corresponding to a combinatorial type where $x_{i}$ and $x_{j}$ are adjacent to the same 3 -valent vertex $V$. All ridges in $\mathcal{M}_{n+1}$ mapping onto $\sigma^{\prime}$, are obtained by attaching $x_{0}$ to any of the vertices. If not attached to $V$, the induced partition $A, B, C, D$ cannot separate $i$ and $j$. If attached to $V$, the induced partition is $\{0\},\{i\},\{j\}, D$. It follows from $\{0, i\} \neq I$ and $\{0, j\} \neq J$ that $D$ intersects both $I$ and $J$ and, therefore, none of these types is contained in $\operatorname{div}\left(\varphi_{I \mid J}\right)$. Hence, $\sigma^{\prime}$ is not contained in the push-forward of $\operatorname{div}\left(\varphi_{I \mid J}\right)$. But $\mathcal{M}_{n}$ is irreducible, thus $\mathrm{ft}_{0 *}\left(\operatorname{div}\left(\varphi_{I \mid J}\right)\right)=0$.

Lemma 2.24. For $n \geq 4$, we define

$$
\left(x_{1} \mid x_{2}, x_{3}\right):=\sum_{\substack{I \mid J \\ 1 \in I \\ 2,3 \in J}} \operatorname{div}\left(\varphi_{I \mid J}\right) .
$$

Then

$$
\operatorname{div}\left(\psi_{1}\right)=\left(x_{1} \mid x_{2}, x_{3}\right) .
$$

Proof. We use induction on the number of leaves $n$. For $n=4$, only the partition $\{1,4\} \mid\{2,3\}$ contributes to the sum. But $\operatorname{div}\left(\psi_{1}\right)$ as well as $\operatorname{div}\left(\varphi_{1,4 \mid 2,3}\right)$ is just the single vertex in $\mathcal{M}_{4}$ parametrizing the curve ${ }_{4}^{1} \times{ }_{3}^{2}$ with weight 1 . For the induction step, assume $n \geq 4$, and consider the morphism $\mathrm{ft}_{0}: \mathcal{M}_{n+1} \rightarrow \mathcal{M}_{n}$ that forgets the leaf $x_{0}$. Let $I^{\prime} \mid J^{\prime}$ be a partition of $[n]$. Then $\mathrm{ft}_{0}^{*}\left(\varphi_{I^{\prime} \mid J^{\prime}}\right)$ measures the sum of the lengths of the edges separating $I^{\prime}$ and $J^{\prime}$ if present. Hence, we obtain

$$
\mathrm{ft}_{0}^{*}\left(\varphi_{I^{\prime} \mid J^{\prime}}\right)=\varphi_{I^{\prime} \cup\{0\} \mid J^{\prime}}+\varphi_{I^{\prime} \mid J^{\prime} \cup\{0\}} .
$$

Using the induction hypothesis, we conclude that $\mathrm{ft}_{0}^{*}\left(\psi_{1}\right)$ equals the sum on the right hand side except for the partition $\{0,1\} \mid\{0,1\}^{c}$. This missing summand is provided by Lemma 2.16.

Lemma 2.25. Let $n \geq 4$, and let $\mathrm{ft}_{0}: \mathcal{M}_{n+1} \rightarrow \mathcal{M}_{n}$ be the morphism that forgets the leaf $x_{0}$. Then

$$
\mathrm{ft}_{0 *}\left(\operatorname{div}\left(\psi_{0}\right)\right)=(n-2) \mathcal{M}_{n} .
$$


Proof. We express $\psi_{0}$ as $\left(x_{0} \mid x_{1}, x_{2}\right)$ by Lemma 2.24 and use linearity of the push-forward. Lemma 2.23 says that we get one $\mathcal{M}_{n}$ for each $\varphi_{\{0, k\} \mid\{0, k\} c}$ and zero for each other $\varphi_{I \mid J}$. As $k$ runs through $\{3, \ldots, n\}$, the statement follows.

Proposition 2.26 (Dilaton equation for abstract curves). Let

$$
\left\langle\prod_{k=1}^{n} \tau_{a_{k}}\right\rangle
$$

be a zero-dimensional intersection product. Then

$$
\left\langle\tau_{1} \cdot \prod_{k=1}^{n} \tau_{a_{k}}\right\rangle=(n-2)\left\langle\prod_{k=1}^{n} \tau_{a_{k}}\right\rangle .
$$

Proof. The proof is identical to the algebro-geometric one, using Lemma 2.15, Corollary 2.17, Lemma 2.18, Lemma 2.25 and the projection formula.

As degree is preserved, we push forward $\left(\tau_{1} \cdot \prod_{k=1}^{n} \tau_{a_{k}}\right)$ along the forgetful morphism $\mathrm{ft}_{0}$ forgetting the extra leaf $x_{0}$ corresponding to the factor $\tau_{1}$. To see what happens, we use Corollary 2.17 (b) and replace each term $\psi_{k}^{a_{k}}$ by $\mathrm{ft}_{0}^{*}\left(\psi_{k}\right)^{a_{k}}+\mathrm{ft}_{0}^{*}\left(\psi_{k}\right)^{a_{k}-1} \cdot \varphi_{0, k}$. When we multiply the whole product out, all summands containing a factor $\varphi_{0, k}$ vanish when multiplied with $\psi_{0}$ (see Lemma 2.15). It follows that

$$
\psi_{0} \cdot \prod_{k=1}^{n} \psi_{k}^{a_{k}}=\psi_{0} \cdot \prod_{k=1}^{n} \mathrm{ft}_{0}^{*}\left(\psi_{k}\right)^{a_{k}},
$$

and the projection formula together with $\mathrm{ft}_{0 *}\left(\operatorname{div}\left(\psi_{0}\right)\right)=(n-2) \mathcal{M}_{n}$ from Lemma 2.25 gives the desired result.

3. Intersections on the space of parametrized curves. In the previous section, we proved that the tropical 'boundary divisors' and $\Psi$-divisors satisfy exactly the same intersection-theoretic formulas as their classical counterparts. We did this using purely tropical arguments but could have instead used tropicalization methods and toric intersection theory (cf., [13]). The situation changes as we move on to curves together with maps to $\mathbb{R}^{n}$. In this case, as $\bar{M}_{0, n}\left(\mathbb{P}^{n}, d\right)$ 
does not admit a nice toric embedding, therefore the tropicalization of its intersection ring is not yet well understood. (In fact, we will see that, at least with our definitions, the intersection theories are not completely identical.) So at least, from now on, we are somehow forced to take this purely tropical approach. Let us start with the necessary definitions.

A (labeled) degree $\Delta$ in $\mathbb{R}^{r}$ is a finite set of labels together with a map $\Delta \rightarrow \mathbb{Z}^{r} \backslash\{0\}$ to the set of non-zero integer vectors. Furthermore, the images of this map are denoted by $v\left(x_{i}\right), i \in \Delta$, as they will later play the role of the directions of the leaves $x_{i}$, sum up to zero, i.e., $\sum_{i \in \Delta} v\left(x_{i}\right)=0$. The number of elements in $\Delta$ is denoted by $\# \Delta$ (to distinguish it from the support of a cycle). As an example, we define the projective degree $d$ (in dimension $r$ ) to be the set $[(r+1) d]$ with the map

$$
\begin{aligned}
{[(r+1) d] } & \longrightarrow \mathbb{Z}^{r} \backslash\{0\}, \\
1, \ldots, d & \longmapsto-e_{0}, \\
d+1, \ldots, 2 d & \longmapsto-e_{1}, \\
: & \\
r d+1, \ldots,(r+1) d & \longmapsto-e_{r},
\end{aligned}
$$

where, as usual, $e_{1}, \ldots, e_{r}$ denote the standard basis vectors and $e_{0}:=e_{1}+\ldots+e_{r}$.

Definition 3.1. An n-marked (labeled) parametrized curve of degree $\Delta$ in $\mathbb{R}^{r}$ is a tuple $(C, h)$, where $C$ is an $[n] \cup \Delta$-marked smooth abstract curve and $h: C \rightarrow \mathbb{R}^{r}$ is a tropical morphism such that, for all leaves $x_{i}$, the ray $h\left(x_{i}\right) \subseteq \mathbb{R}^{r}$ has direction $v\left(x_{i}\right)$. Here, $v\left(x_{i}\right)$ is set to be zero if $i \in[n]$ (i.e., the marked leaves $x_{i}, i \in[n]$ are contracted to a point). The genus of $(C, h)$ is defined to be the genus of $C$.

Remark 3.2. The leaves $x_{i}, i \in[n]$ are called marked leaves, as they correspond to the marked points of stable maps classically. Marked leaves are contracted by $h$. In contrast to that, we call the leaves $x_{i}$, $i \in \Delta$ non-contracted leaves. Our curves are called "labeled" as the non-contracted leaves are also labeled. 
Two parametrized curves $(C, h)$ and $\left(C^{\prime}, h^{\prime}\right)$ are called isomorphic (and therefore identified in the following) if there exists an isomorphism $\Phi: C \rightarrow C^{\prime}$ identifying the labels and satisfying $h=h^{\prime} \circ \Phi$.

Let us compare our definition to [8, Definition 4.1]. Conditions (a) and (b) in that definition make sure that $h$ is a tropical morphism in our sense (at least locally; but again, considering the family property of $\mathrm{ft}_{0}, \mathrm{ev}_{0}$ over $\mathcal{M}_{n}^{\mathrm{lab}}\left(\mathbb{R}^{r}, \Delta\right)$ we will see that a global integer affine map $h$ always exists). Condition (c) is also contained in our definition.

Let $\mathcal{M}_{n}^{\text {lab }}\left(\mathbb{R}^{r}, \Delta\right)$ be the moduli space of rational $n$-marked labeled parametrized curves of degree $\Delta$ in $\mathbb{R}^{r}$. Its construction as a tropical cycle can be found in [8, subsection 4.7]. After fixing one of the marked leaves $x_{i}$ as an anchor leaf (we avoid "root leaf" as, from the botanic point of view, this does not make much sense), we can identify $\mathcal{M}_{n}^{\text {lab }}\left(\mathbb{R}^{r}, \Delta\right)$ with $\mathcal{M}_{[n] \cup \Delta} \times \mathbb{R}^{r}$, where the first factor parametrizes the abstract curve $C$ and the second factor contains the coordinates of the image point of the anchor leaf $x_{i}$. As our curves are rational, this suffices to fix the morphism $h$. Indeed, as $\Delta$ determines the directions of all leaves, we can use the balancing condition to recursively compute the directions of all bounded edges as well. Hence, $h$ is uniquely determined by the lengths of the edges and the coordinates of one image point (in our case, $h\left(x_{i}\right)$ ).

So, again, cones in $\mathcal{M}_{n}^{\text {lab }}\left(\mathbb{R}^{r}, \Delta\right)$ correspond to combinatorial types of the underlying abstract curves, but this time the minimal cone is not zero- but $r$-dimensional because we can move the curve in $\mathbb{R}^{r}$.

For enumerative purposes, we would like to identify curves whose only difference is the labeling of the non-contracted leaves. Let $\mathcal{M}_{n}\left(\mathbb{R}^{r}, \Delta\right)$ denote the set of these unlabeled curves. Then the number of elements in a general fibre of the map $\mathcal{M}_{n}^{\text {lab }}\left(\mathbb{R}^{r}, \Delta\right) \rightarrow \mathcal{M}_{n}\left(\mathbb{R}^{r}, \Delta\right)$ forgetting the labeling of the non-contracted leaves equals the number of possibilities to label a general unlabeled curve, which is

$$
\Delta !:=\prod_{v \in \mathbb{Z}^{r} \backslash\{0\}} n(v) !,
$$

where $n(v)$ denotes the number of times $v$ occurs as $v\left(x_{i}\right), i \in \Delta$. 
Therefore, each enumerative invariant computed on $\mathcal{M}_{n}^{\text {lab }}\left(\mathbb{R}^{r}, \Delta\right)$ must simply be divided by $\Delta$ ! to get the corresponding one in $\mathcal{M}_{n}\left(\mathbb{R}^{r}, \Delta\right)$.

From now on, $I \mid J$ denotes a (non-empty) partition of $[n] \cup \Delta$ (or $\{0\} \cup[n] \cup \Delta$ if we work with $\left.\mathcal{M}_{n+1}^{\text {lab }}\left(\mathbb{R}^{r}, \Delta\right)\right)$. Again, such partitions can be used as global labels of the edges of our curves. The direction of the image of the corresponding edge under $h$ is given by

$$
v_{I \mid J}:=\sum_{i \in I} v\left(x_{i}\right)=-\left(\sum_{j \in J} v\left(x_{j}\right)\right)
$$

(as an exception, the ordering of $I$ and $J$ plays a little role here, namely, $v_{I \mid J}=-v_{J \mid I}$ ). We call $I \mid J$ reducible if $v_{I \mid J}=0$ (i.e., if the corresponding edge is contracted). This is equivalent to requiring that the corresponding split sets $\Delta_{I}=I \cap \Delta$ and $\Delta_{J}=J \cap \Delta$ fulfill the balancing condition, i.e., are degrees on their own. Also the marked leaves split up into $[n]=\left\{i \in I \mid v\left(x_{i}\right)=0\right\} \cup\left\{j \in I \mid v\left(x_{j}\right)=0\right\}$. In this sense, the partition corresponds (nearly) to a conventional partition $\left(S^{\prime}, \beta^{\prime} \mid S^{\prime \prime}, \beta^{\prime \prime}\right)$ of the marked points $S=S^{\prime} \cup S^{\prime \prime}$ and the degree $\beta=\beta^{\prime}+\beta^{\prime \prime}$, occurring for example in the classical splitting lemma. However, note that, in the tropical setting, it is possible to permute non-contracted leaves with the same direction vector between $I$ and $J$ without changing the corresponding conventional partition; hence, in general, several tropical reducible partitions correspond to the same conventional partition. The non-reducible partitions $I \mid J$ (i.e., $v_{I \mid J} \neq 0$ ) do not correspond to such a partition.

There exists a forgetful map $\mathrm{ft}^{\prime}: \mathcal{M}_{n}^{\text {lab }}\left(\mathbb{R}^{r}, \Delta\right) \rightarrow \mathcal{M}_{[n] \cup \Delta}$ forgetting just the position of a curve in $\mathbb{R}^{r}$. This forgetful map $\mathrm{ft}^{\prime}$ : $\mathcal{M}_{n}^{\text {lab }}\left(\mathbb{R}^{r}, \Delta\right) \rightarrow \mathcal{M}_{[n] \cup \Delta}$ is a morphism of tropical varieties, as after choosing a anchor leaf and identifying $\mathcal{M}_{n}^{\text {lab }}\left(\mathbb{R}^{r}, \Delta\right)$ with $\mathcal{M}_{[n] \cup \Delta} \times \mathbb{R}^{r}$, $\mathrm{ft}^{\prime}$ is just the projection onto the first factor. We use this to define $\Psi$-functions on $\mathcal{M}_{n}^{\text {lab }}\left(\mathbb{R}^{r}, \Delta\right)$.

Definition 3.3 ( $\Psi$-functions for parametrized curves). For a partition $I \mid J$ of $[n] \cup \Delta$ we define the function $\varphi_{I \mid J}$ on $\mathcal{M}_{n}^{\text {lab }}\left(\mathbb{R}^{r}, \Delta\right)$ to be $\mathrm{ft}^{\prime *}\left(\varphi_{I \mid J}^{\mathrm{abstr}}\right)$, where $\varphi_{I \mid J}^{\mathrm{abstr}}$ is the corresponding function on $\mathcal{M}_{[n] \cup \Delta}$. 
For $i=1, \ldots, n$, we define the $k$ th $\Psi$-function on $\mathcal{M}_{n}^{\text {lab }}\left(\mathbb{R}^{r}, \Delta\right)$ to be $\psi_{k}:=\mathrm{ft}^{*}\left(\psi_{k}^{\text {abstr }}\right)$, where the $\psi_{k}^{\text {abstr }}$ is the $k$ th $\Psi$-function on $\mathcal{M}_{[n] \cup \Delta}$.

Remark 3.4. Again, in spite of defining functions, we are actually interested in the divisors. Note that, by Proposition 1.12, the pullbacks of the respective divisors do not depend on the particular functions. Note that, in [17, Definition 2.2], we used the notation $\psi_{k}$ for the divisor instead of the rational function.

We can immediately generalize Theorem 2.14 to parametrized curves (cf., [17, Lemma 2.4]).

Proposition 3.5 (Intersections of $\Psi$-divisors for parametrized curves). Let $a_{1}, \ldots, a_{n}$ be positive integers, and let $X=\prod_{k=1}^{n} \psi_{k}^{a_{k}} \cdot \mathcal{M}_{n}^{\mathrm{lab}}\left(\mathbb{R}^{r}, \Delta\right)$ be a product of $\Psi$-divisors. Then $X$ is the subfan of $\mathcal{M}_{n}^{\text {lab }}\left(\mathbb{R}^{r}, \Delta\right)$ consisting of the closure of the cones of dimension $n+\# \Delta-3-\sum_{i=1}^{n} a_{i}$ whose interior curves $C$ have the following property.

Let $k_{1}, \ldots, k_{q} \subseteq[n]$ be the marked leaves adjacent to a vertex $V$ of $C$. Then the valence of $V$ is

$$
\operatorname{val}(V)=a_{k_{1}}+\cdots+a_{k_{q}}+3
$$

Let us define the multiplicity of this vertex to be $\operatorname{mult}(V):=\left(\begin{array}{c}\operatorname{val}(V)-3 \\ a_{k_{1}}, \ldots, a_{k_{q}}\end{array}\right)$. Then the weight of such a cone $\sigma$ in $X$ is:

$$
\omega_{X}(\sigma)=\prod_{V} \operatorname{mult}(V)
$$

where the product runs through all vertices $V$ of an interior curve of $\sigma$.

Proof. Choose an anchor leaf, and identify $\mathcal{M}_{n}^{\text {lab }}\left(\mathbb{R}^{r}, \Delta\right)$ with $\mathcal{M}_{[n] \cup \Delta} \times \mathbb{R}^{r}$. Then $\mathrm{ft}^{\prime}$ is just the projection on the first factor and we can apply [1, subsection 9.6], i.e., instead of intersecting the pullbacks of the $f_{k}$ on the product, we can just intersect the $f_{k}$ on the first factor and then multiply with $\mathbb{R}^{2}$. Thus,

$$
X=\left(\prod_{k=1}^{n}\left(\psi_{k}^{\text {abstr }}\right)^{a_{k}} \cdot \mathcal{M}_{[n] \cup \Delta}\right) \times \mathbb{R}^{r},
$$


where here $\psi_{k}^{\text {abstr }}$ denotes a $\Psi$-function on $\mathcal{M}_{[n] \cup \Delta}$. Now, as the weight of $\mathbb{R}^{r}$ is one and the combinatorics of a curve do not change under $\mathrm{ft}^{\prime}$, the statements follow from Theorem 2.14.

Proposition 3.6. Let $\mathrm{ft}_{0}$ be the map $\mathcal{M}_{n+1}^{\mathrm{lab}}\left(\mathbb{R}^{r}, \Delta\right) \rightarrow \mathcal{M}_{n}^{\mathrm{lab}}\left(\mathbb{R}^{r}, \Delta\right)$ that forgets the extra leaf $x_{0}$, and assume $n+\# \Delta \geq 4$ (and $n \geq 1$ ). Furthermore, let $x_{i}, x_{j}$ and $x_{k}$ be pairwise different leaves. Then the following equations hold (where all the occurring intersection products are computed in $\mathcal{M}_{n}^{\text {lab }}\left(\mathbb{R}^{r}, \Delta\right)$ or $\mathcal{M}_{n+1}^{\text {lab }}\left(\mathbb{R}^{r}, \Delta\right)$, respectively):

(a) $\varphi_{i, j} \cdot \varphi_{i, k}=0$.

(b) $\varphi_{i, j} \cdot \psi_{i}=0$.

(c) $\operatorname{div}\left(\psi_{k}\right)=\operatorname{div}\left(\mathrm{ft}_{0}^{*} \psi_{k}\right)+\operatorname{div}\left(\varphi_{0, k}\right)$.

(d) $\varphi_{0, k}^{2}=-\mathrm{ft}_{0}^{*}\left(\psi_{k}\right) \cdot \varphi_{0, k}$.

(e) $\psi_{k}^{a}=\mathrm{ft}_{0}^{*}\left(\psi_{k}\right)^{a}+\mathrm{ft}_{0}^{*}\left(\psi_{k}\right)^{a-1} \cdot \varphi_{0, k}$.

(f) $\psi_{k}^{a}=\mathrm{ft}_{0}^{*}\left(\psi_{k}\right)^{a}+(-1)^{a-1} \varphi_{0, k}^{a}$.

(g) $\mathrm{ft}_{0 *}\left(\operatorname{div}\left(\varphi_{0, k}\right)\right)=\mathrm{ft}_{0 *}\left(\operatorname{div}\left(\psi_{k}\right)\right)=\mathcal{M}_{n}^{\mathrm{lab}}\left(\mathbb{R}^{r}, \Delta\right)$.

(h)

$$
\mathrm{ft}_{0 *}\left(\operatorname{div}\left(\varphi_{I \mid J}\right)\right)= \begin{cases}\mathcal{M}_{n}^{\mathrm{lab}}\left(\mathbb{R}^{r}, \Delta\right) & \text { if } I=\{0, k\} \text { or } J=\{0, k\} \\ 0 & \text { for some } k \in[n], \\ 0 & \text { otherwise. }\end{cases}
$$

(i) $\operatorname{div}\left(\psi_{i}\right)=\left(x_{i} \mid x_{j}, x_{k}\right):=\sum_{\substack{i \mid J \\ j \in I \\ j, k \in J}} \operatorname{div}\left(\varphi_{I \mid J}\right)$, where the sum also runs through non-reducible partitions.

(j) $\mathrm{ft}_{0 *}\left(\operatorname{div}\left(\psi_{0}\right)\right)=(n+\# \Delta-2) \mathcal{M}_{n}^{\mathrm{lab}}\left(\mathbb{R}^{r}, \Delta\right)$, (which is different from the algebro-geometric factor $n-2$ that equals the abstract case).

Proof. As in the proof of Proposition 3.5, we apply [1, subsection 9.6] to the morphism $\mathrm{ft}^{\prime}: \mathcal{M}_{n}^{\mathrm{lab}}\left(\mathbb{R}^{r}, \Delta\right)=\mathcal{M}_{[n] \cup \Delta} \times \mathbb{R}^{r} \rightarrow \mathcal{M}_{[n] \cup \Delta}$, forgetting the position in $\mathbb{R}^{r}$. This means that, instead of computing the intersection product on $\mathcal{M}_{n}^{\text {lab }}\left(\mathbb{R}^{r}, \Delta\right)$, we can compute them on $\mathcal{M}_{[n] \cup \Delta}$ and therefore use the corresponding statements for abstract curves. For statements $\mathrm{c}-\mathrm{h}$ and $\mathrm{j}$, we also use $\mathrm{ft}_{0}=\mathrm{ft}_{0}^{\mathrm{abstr}} \times \mathrm{id}$.

Definition 3.7 (Evaluation maps and their pull-backs). The evaluation map $\operatorname{ev}_{k}: \mathcal{M}_{n}^{\mathrm{lab}}\left(\mathbb{R}^{r}, \Delta\right) \rightarrow \mathbb{R}^{r}$, for $k \in[n]$, maps each 
parametrized curve $(C, h)$ to the position of its $k$ th leaf $h\left(x_{k}\right)$ (see [8, subsection 4.2]). If we choose one of the marked leaves, say $x_{a}$, as an anchor leaf, then the evaluation maps are morphisms from $\mathcal{M}_{[n] \cup \Delta} \times \mathbb{R}^{r}$ to $\mathbb{R}^{r}$ obeying the following mapping rule:

$$
\left(C^{\mathrm{abstr}}, P\right) \longmapsto P+\sum_{\substack { I \mid J \\
\begin{subarray}{c}{a \in I \\
k \in J{ I | J \\
\begin{subarray} { c } { a \in I \\
k \in J } }\end{subarray}} \varphi_{I \mid J}\left(C^{\mathrm{abstr}}\right) v_{I \mid J}
$$

In particular, if our anchor leaf is chosen to be $x_{k}$, then $\mathrm{ev}_{k}$ is just the projection onto the second factor. Let $C \in Z_{m}^{\text {c.i. }}\left(\mathbb{R}^{r}\right)$ be given by $C=h_{1} \cdot \ldots \cdot h_{l} \cdot X$. Then we can apply Proposition 1.12 which states that there is a well-defined pull-back of $C$ along $\mathrm{ev}_{k}$

$$
\operatorname{ev}_{k}^{*}(C):=\operatorname{ev}_{k}^{*}\left(h_{1}\right) \cdot \ldots \cdot \operatorname{ev}_{k}^{*}\left(h_{l}\right) .
$$

Proposition 3.8 (Family property of $\mathrm{ft}_{0}, \mathrm{ev}_{0}$ for parametrized curves). Let $p$ be a point in $\mathcal{M}_{n}^{\text {lab }}\left(\mathbb{R}^{r}, \Delta\right)$, and let $C_{p}=\mathrm{ft}_{0}^{-1}(p)$ be the fibre of $p$ under the forgetful morphism $\mathrm{ft}_{0}: \mathcal{M}_{n+1}^{\text {lab }}\left(\mathbb{R}^{r}, \Delta\right) \rightarrow \mathcal{M}_{n}^{\text {lab }}\left(\mathbb{R}^{r}, \Delta\right)$. Then the following hold:

(i) When we equip all its facets with weight $1, C_{p}$ is a rational smooth abstract curve. Its leaves are naturally $[n] \cup \Delta$-marked by $L_{i}:=\overline{\left\{y \in C_{p} \mid \varphi_{0, i}(y)>0\right\}}$.

(ii) The tuple $\left(C_{p},\left.\mathrm{ev}_{0}\right|_{\left|C_{p}\right|}\right)$ is an n-marked parametrized curve of degree $\Delta$. Moreover, $p$ represents $\left(C_{p}\right.$, ev $\left.\left.\right|_{\left|C_{p}\right|}\right)$.

(iii) Let $\sum_{k} \mu_{k} p_{k}=\varphi_{1} \cdot \ldots \cdot \varphi_{n+\# \Delta-3} \cdot \mathcal{M}_{n}^{\text {lab }}\left(\mathbb{R}^{r}, \Delta\right)$ be a zerodimensional cycle in $\mathcal{M}_{n}^{\text {lab }}\left(\mathbb{R}^{r}, \Delta\right)$ obtained as the intersection product of convex functions $\varphi_{j}$. Then

$$
\mathrm{ft}_{0}^{*}\left(\varphi_{1}\right) \cdot \ldots \cdot \mathrm{ft}_{0}^{*}\left(\varphi_{n+\# \Delta-3}\right) \cdot \mathcal{M}_{n+1}^{\mathrm{lab}}\left(\mathbb{R}^{r}, \Delta\right)=\sum_{k} \mu_{k} C_{p_{k}} .
$$

We write this as

$$
\mathrm{ft}_{0}^{*}\left(\sum_{k} \mu_{k} p_{k}\right)=\sum_{k} \mu_{k} C_{p_{k}}
$$




\section{Proof.}

(i) First of all, let us fix an anchor leaf $x_{a}, a \in[n]$ in order to identify $\mathcal{M}_{n+1}^{\text {lab }}\left(\mathbb{R}^{r}, \Delta\right)=\mathcal{M}_{n+\# \Delta+1} \times \mathbb{R}^{r}$ and $\mathcal{M}_{n}^{\text {lab }}\left(\mathbb{R}^{r}, \Delta\right)=$ $\mathcal{M}_{[n] \cup \Delta} \times \mathbb{R}^{r}$. We again use $\mathrm{ft}_{0}=\mathrm{ft}_{0}^{\text {abstr }} \times \mathrm{id}$, where $\mathrm{ft}_{0}^{\text {abstr }}$ is the corresponding forgetful map on abstract spaces. Then the fibre of $p=\left(p^{\prime}, P\right)$ equals $C_{p^{\prime}} \times\{P\}$, where $C_{p^{\prime}}$ is the $[n] \cup \Delta$-marked rational smooth abstract curve considered in Proposition 2.19 (i)-(iii).

(ii) We have to check that the direction of the $\operatorname{rays} \operatorname{ev}_{0}\left(L_{i}\right)$ are correct. For curves in $L_{i}$, the only length that varies is that of the third edge adjacent to the same three-valent vertex as $x_{i}$ and $x_{0}$. Hence, we can use the description of $\mathrm{ev}_{0}$ in Definition 3.7 and obtain, for all $y \in L_{i}$,

$$
\left.\mathrm{ev}_{0}\right|_{L_{i}}(y)=Q+\varphi_{0, i}(y) \cdot v_{\{0, i\} \mid\{0, i\}^{c}},
$$

where $Q \in \mathbb{R}^{r}$ is some constant vector. But $v_{\{0, i\} \mid\{0, i\}^{c}}=$ $v\left(x_{i}\right)+v\left(x_{0}\right)=v\left(x_{i}\right)$ is the expected direction.

To show that $p=\left(p^{\prime}, P\right)$ represents $\left(C_{p},\left.\mathrm{ev}_{0}\right|_{\left|C_{p}\right|}\right)$, it actually suffices to prove that the anchor leaf $L_{a}$ of $C_{p}$ is mapped to the point $P$ under $\mathrm{ev}_{0}$, which is obviously the case as $\left.\mathrm{ev}_{0}\right|_{L_{a}}=\left.\mathrm{ev}_{a}\right|_{L_{a}}$, and $\mathrm{ev}_{a}$ is just the projection on the second factor of $C_{p^{\prime}} \times\{P\}$.

We can literally use the same proof as in the abstract case Proposition 2.19 (iv) using Proposition $3.6 \mathrm{~g}$.

Notation 3.9 (Tropical Gromov-Witten invariants). Let us now extend our $\tau$-notation to the case of parametrized curves. For any positive integers $a_{1}, \ldots, a_{n}$ and complete intersection cycles $C_{1}, \ldots, C_{n}$ $\in Z_{*}^{\text {c.i. }}\left(\mathbb{R}^{r}\right)$, we define

$$
\begin{aligned}
\left(\tau_{a_{1}}\left(C_{1}\right) \cdot \ldots \cdot\right. & \left.\tau_{a_{n}}\left(C_{n}\right)\right)_{\Delta}^{\mathbb{R}^{r}} \\
& :=\psi_{1}^{a_{1}} \cdot \operatorname{ev}_{1}^{*}\left(C_{1}\right) \cdot \ldots \cdot \psi_{n}^{a_{n}} \cdot \mathrm{ev}_{n}^{*}\left(C_{n}\right) \cdot \mathcal{M}_{n}^{\mathrm{lab}}\left(\mathbb{R}^{r}, \Delta\right) .
\end{aligned}
$$

Once again, each factor $\tau_{a_{k}}\left(C_{k}\right)$ stands for a marked leaf subject to $a_{k} \Psi$-conditions and to the condition that it must meet $C_{k}$. Let $c_{k}$ be the codimension of $C_{k}$ in $\mathbb{R}^{r}$. If $\sum\left(a_{k}+c_{k}\right)=\operatorname{dim}\left(\mathcal{M}_{n}^{\mathrm{lab}}\left(\mathbb{R}^{r}, \Delta\right)\right)=$ $n+\# \Delta+r-3$, the above cycle is zero-dimensional, and we denote 
its degree by

$$
\left\langle\tau_{a_{1}}\left(C_{1}\right) \cdot \ldots \cdot \tau_{a_{n}}\left(C_{n}\right)\right\rangle_{\Delta}^{\mathbb{R}^{r}}
$$

These numbers are called tropical descendant Gromov-Witten invariants. In [17], these numbers were studied in the cases $r=2, \Delta=d$, and all $C_{i}$ are tropical lines.

Remark 3.10 (Enumerative meaning of tropical Gromov-Witten invariants). Let $\left(\tau_{a_{1}}\left(C_{1}\right) \cdot \ldots \cdot \tau_{a_{n}}\left(C_{n}\right)\right)$ be an intersection product as defined above. If we set $X=\prod_{k=1}^{n} \psi_{k}^{a_{k}} \cdot \mathcal{M}_{n}^{\text {lab }}\left(\mathbb{R}^{r}, \Delta\right)$ and apply Proposition 1.15 to the morphisms ev $\mathrm{v}_{k}: X \rightarrow \mathbb{R}^{r}$, we can conclude the following (as discussed in Remark 1.16). After replacing all the cycles $C_{k}$ by general translations (called general conditions in the following), $\left.Z:=\tau_{a_{1}}\left(C_{1}\right) \cdot \ldots \cdot \tau_{a_{n}}\left(C_{n}\right)\right)$ is the set of curves $C$ such that

- every vertex $V \in C$ with adjacent marked leaves $k_{1}, \ldots, k_{q}$ fulfills

$$
\operatorname{val}(V) \geq a_{k_{1}}+\ldots+a_{k_{q}}+3
$$

- for all $k=1, \ldots, n$, the following holds:

$$
\operatorname{ev}_{k}(C) \in C_{k}
$$

Additionally, the facets of $Z$ (i.e., general curves) are equipped with (possibly zero) weights.

Moreover, assume that all the cycles $C_{k}$ can be described by convex functions $h_{1} \cdots h_{l} \cdot \mathbb{R}^{r}$. Then, by Proposition 1.10, all these weights are positive (in particular, $|Z|$ really is the set of such curves). Thus, if $Z$ is zero-dimensional, $\operatorname{deg}(Z)=\left\langle\tau_{a_{1}}\left(C_{1}\right) \cdot \ldots \cdot \tau_{a_{n}}\left(C_{n}\right)\right\rangle$ is the number of curves satisfying the above properties, counted with a certain integer multiplicity/weight. Now, again, if all $C_{k}$ can be described by convex functions, all these multiplicities and, in particular, $\left\langle\tau_{a_{1}}\left(C_{1}\right) \cdot \ldots \cdot \tau_{a_{n}}\left(C_{n}\right)\right\rangle$ are positive.

Let $\mathrm{ft}_{0}: \mathcal{M}_{n+1}^{\mathrm{lab}}\left(\mathbb{R}^{r}, \Delta\right) \rightarrow \mathcal{M}_{n}^{\mathrm{lab}}\left(\mathbb{R}^{r}, \Delta\right)$ be the morphism that forgets the leaf $x_{0}$. Then, by abuse of notation, the equation,

$$
\mathrm{ft}_{0}^{*}\left(\mathrm{ev}_{k}\right)=\mathrm{ev}_{k}
$$


holds for all $k \in[n]$. This equality directly implies the following extensions of the string and dilaton equation to the case of parametrized curves.

Theorem 3.11 (String equation for parametrized curves). Let

$$
\left(\tau_{0}\left(\mathbb{R}^{r}\right) \cdot \prod_{k=1}^{n} \tau_{a_{k}}\left(C_{k}\right)\right)_{\Delta}
$$

be a zero-dimensional cycle. Then

$$
\left\langle\tau_{0}\left(\mathbb{R}^{r}\right) \cdot \prod_{k=1}^{n} \tau_{a_{k}}\left(C_{k}\right)\right\rangle_{\Delta}=\sum_{k=1}^{n}\left\langle\tau_{a_{k}-1}\left(C_{k}\right) \cdot \prod_{l \neq k} \tau_{a_{l}}\left(C_{l}\right)\right\rangle_{\Delta} .
$$

Theorem 3.12 (Dilaton equation for parametrized curves). Let $\left(\tau_{1}\left(\mathbb{R}^{r}\right) \cdot \prod_{k=1}^{n} \tau_{a_{k}}\left(C_{k}\right)\right)_{\Delta}$ be a zero-dimensional cycle. The following equation holds:

$$
\left\langle\tau_{1}\left(\mathbb{R}^{r}\right) \cdot \prod_{k=1}^{n} \tau_{a_{k}}\left(C_{k}\right)\right\rangle_{\Delta}=(n+\# \Delta-2)\left\langle\prod_{k=1}^{n} \tau_{a_{k}}\left(C_{k}\right)\right\rangle_{\Delta} .
$$

Proofs. In both cases, the proofs are completely analogous to the abstract case using Proposition 3.6 and $\mathrm{ft}_{0}^{*}\left(\mathrm{ev}_{k}\right)=\mathrm{ev}_{k}$.

Remark 3.13. Note that the factor appearing in the dilaton equation is different from the algebro-geometric one, due to $\mathrm{ft}_{0 *}\left(\psi_{0}\right)=(n+$ $\# \Delta-2) \cdot \mathcal{M}_{n}^{\text {lab }}\left(\mathbb{R}^{r}, \Delta\right)$ (cf., Proposition $\left.3.6(\mathrm{j})\right)$.

Lemma 3.14. Let $h$ be a rational function. Then

$$
\operatorname{ev}_{k}^{*}(h) \cdot \varphi_{k, l} \cdot \mathcal{M}_{n}^{\mathrm{lab}}\left(\mathbb{R}^{r}, \Delta\right)=\mathrm{ev}_{l}^{*}(h) \cdot \varphi_{k, l} \cdot \mathcal{M}_{n}^{\mathrm{lab}}\left(\mathbb{R}^{r}, \Delta\right) .
$$

Proof. In all curves corresponding to points in $\operatorname{div}\left(\varphi_{k, l}\right)$, the leaves $k$ and $l$ lie at a common vertex. Therefore, their coordinates in $\mathbb{R}^{r}$ must agree, which means $\left.\operatorname{ev}_{k}\right|_{\left|\operatorname{div}\left(\varphi_{k, l}\right)\right|}=\left.\operatorname{ev}_{l}\right|_{\left|\operatorname{div}\left(\varphi_{k, l}\right)\right|}$. The result follows.

For a given labeled degree $\Delta$, we define $\delta(\Delta)$ to be the associated unlabeled degree in the sense of subsection 1.1: $\delta(\Delta)$ is the one- 
dimensional balanced fan in $\mathbb{R}^{r}$ consisting of all the rays generated by the direction vectors $v_{k}, k \in \Delta$, appearing in $\Delta$. The weight of such a ray $\mathbb{R}_{\geq} v$, where $v$ is primitive, is given by

$$
\sum_{\substack{k \in \Delta \\ v_{k} \in \mathbb{Z}_{>0} v}}\left|\mathbb{Z} v / \mathbb{Z} v_{k}\right| .
$$

Obviously, if $(C, h) \in \mathcal{M}_{n}^{\text {lab }}\left(\mathbb{R}^{r}, \Delta\right)$ is an arbitrary $n$-marked parametrized curve of degree $\Delta$, then, by definition, $\delta(h(C))=\delta(\Delta)$ holds.

For a given rational function $h$ on $\mathbb{R}^{r}$ we define $h \cdot \Delta$ to be $\operatorname{deg}(h \cdot \delta(\Delta))$

Lemma 3.15. Let $h$ be a rational function on $\mathbb{R}^{r}$, and define $Y:=$ $\mathrm{ev}_{0}^{*}(h) \cdot \mathcal{M}_{n+1}^{\mathrm{lab}}\left(\mathbb{R}^{r}, \Delta\right)$. Then

$$
\mathrm{ft}_{0 *}(Y)=(h \cdot \Delta) \mathcal{M}_{n}^{\mathrm{lab}}\left(\mathbb{R}^{r}, \Delta\right) .
$$

Proof. As our moduli space $\mathcal{M}_{n}^{\text {lab }}\left(\mathbb{R}^{r}, \Delta\right)$ is irreducible, we know that $\mathrm{ft}_{0 *}(Y)=\alpha \cdot \mathcal{M}_{n}^{\text {lab }}\left(\mathbb{R}^{r}, \Delta\right)$ for an integer $\alpha$. To compute this number, we set $m:=n+\# \Delta+r-3$ and consider the zero-dimensional intersection product $Z=\varphi_{1} \cdots \varphi_{m} \cdot \mathcal{M}_{n}^{\text {lab }}\left(\mathbb{R}^{r}, \Delta\right)$ of arbitrary convex functions $\varphi_{1}, \ldots, \varphi_{m}$ such that $\operatorname{deg}(Z) \neq 0$ (e.g., $Z=\psi_{1}^{m-r} \cdot \mathrm{ev}_{1}(P)$ for some point $P \in \mathbb{R}^{r}$ ). If we pull back $Z$ along $\mathrm{ft}_{0}$, we know by the projection formula that

$$
\operatorname{deg}\left(\operatorname{ev}_{0}(h) \cdot \mathrm{ft}_{0}^{*}(Z)\right)=\alpha \cdot \operatorname{deg}(Z) .
$$

On the other hand, by the family property of $\mathrm{ft}_{0}$, we know that $Z$ is the union of curves represented by the points in $Z$ (with equivalent weights) and therefore the push-forward $\mathrm{ev}_{0 *}\left(\mathrm{ft}_{0}^{*}(Z)\right)$ is rationally equivalent to its degree

$$
\delta\left(\operatorname{ev}_{0 *}\left(\mathrm{ft}_{0}^{*}(Z)\right)\right)=\operatorname{deg}(Z) \cdot \delta(\Delta) .
$$

So, applying the projection formula to $\mathrm{ev}_{0}$, we obtain

$$
\operatorname{deg}\left(\operatorname{ev}_{0}(h) \cdot \mathrm{ft}_{0}^{*}(Z)\right)=\operatorname{deg}(Z) \cdot(h \cdot \Delta) .
$$

But this implies $h \cdot \Delta=\alpha$, which proves the claim. 
We can now prove the following rather general version of the divisor equation.

Theorem 3.16 (Divisor equation). Let $h$ be a rational function on $\mathbb{R}^{r}$, and let $\left(\prod_{k=1}^{n} \tau_{a_{k}}\left(C_{k}\right)\right)_{\Delta}$ be a one-dimensional cycle. Then

$$
\begin{aligned}
\left\langle\tau_{0}(h) \cdot \prod_{k=1}^{n} \tau_{a_{k}}\left(C_{k}\right)\right\rangle_{\Delta}= & (h \cdot \Delta)\left\langle\prod_{k=1}^{n} \tau_{a_{k}}\left(C_{k}\right)\right\rangle_{\Delta} \\
& +\sum_{k=1}^{n}\left\langle\tau_{a_{k}-1}\left(h \cdot C_{k}\right) \prod_{l \neq k} \tau_{a_{l}}\left(C_{l}\right)\right\rangle_{\Delta} .
\end{aligned}
$$

Proof. First we use Proposition 3.6 (a) and (e). We replace each factor $\psi_{k}^{a_{k}}$ by $\mathrm{ft}_{0}^{*}\left(\psi_{k}\right)^{a_{k}}+\mathrm{ft}_{0}^{*}\left(\psi_{k}\right)^{a_{k}-1} \cdot \varphi_{0, k}$ and multiply out. All terms containing two $\varphi$-factors vanish. In terms with only one factor $\varphi_{0, k}$, we replace $\mathrm{ev}_{0}(h)$ by $\mathrm{ev}_{k}(h)$ using Lemma 3.14. Now we push forward along $\mathrm{ft}_{0}$ and produce the desired equation by applying Lemma 3.15 and $\mathrm{ft}_{0 *}\left(\operatorname{div}\left(\varphi_{0, k}\right)\right)=\mathcal{M}_{n}^{\mathrm{lab}}\left(\mathbb{R}^{r}, \Delta\right)$.

Note that the divisor equation can be used to prove the statement of [17, Proposition 2.10].

4. The splitting lemma. The basic fact used to compute GromovWitten type invariants of $\bar{M}_{g, n}(X, \beta)$ is the recursive structure of its boundary: its irreducible components correspond to reducible curves with a certain partition of the combinatoric data and therefore are (nearly) a product of two "smaller" moduli spaces. In this section, we will investigate how far this principle can be carried over to the tropical world.

\subsection{The case of abstract curves.}

Definition 4.1. Let $S$ be a finite set. By $\mathcal{M}_{S}$, we denote the moduli space of $|S|$-marked tropical curves $\mathcal{M}_{|S|}$ where we label the leaves by elements in $S$. For each partition $I \mid J$ of $[n]$, we construct the map $\rho_{I \mid J}: \mathcal{M}_{I \cup\{x\}} \times \mathcal{M}_{J \cup\{y\}} \rightarrow \varphi_{I \mid J} \cdot \mathcal{M}_{n}$ by the following rule. Given two curves $\left(p_{I}, p_{J}\right) \in \mathcal{M}_{I \cup\{x\}} \times \mathcal{M}_{J \cup\{y\}}$, we remove the extra leaves $x$ and $y$ and glue the curves together at the two vertices to which these 
leaves have been adjacent. In other words, we glue $x$ and $y$ together by creating a bounded edge whose length we define to be 0 . In the coordinates of the space of tree metrics, this map is given by the linear map

$$
\begin{aligned}
& \rho_{I \mid J}: \mathbb{R}^{\left(\begin{array}{l}
I \\
2
\end{array}\right)} \times \mathbb{R}^{\left(\begin{array}{c}
J \\
2
\end{array}\right)} \longrightarrow \mathbb{R}^{\left(\begin{array}{c}
n \\
2
\end{array}\right)}, \\
&\left(p_{I}, p_{J}\right) \longmapsto p,
\end{aligned}
$$

where

$$
p_{k, l}:= \begin{cases}p_{I_{k, l}} & \text { if } k, l \in I, \\ p_{J_{k, l}} & \text { if } k, l \in J, \\ p_{I_{k, x}}+p_{J_{y}, l} & \text { if } k \in I, l \in J .\end{cases}
$$

Caution. This map does not induce a linear map on the corresponding quotients in which our moduli spaces are balanced and therefore $\rho_{I \mid J}$ is not a tropical morphism of our moduli spaces. In addition, $\rho_{I \mid J}$ is not even locally linear around ridges of our moduli spaces considered as balanced complexes in the quotients. On the other hand, $\rho_{I \mid J}$ is at least piecewise linear (i.e., it is linear on all cones of $\left.\mathcal{M}_{I \cup\{x\}} \times \mathcal{M}_{J \cup\{y\}}\right)$. Its image is a polyhedral complex, namely, the positive part of $\varphi_{I \mid J} \cdot \mathcal{M}_{n}$ (i.e., it consists of all (faces of) facets ${ }_{D}^{A} \times{ }_{C}^{B}$ with $A \cup B=I)$.

Definition 4.2 (Morphisms of rational polyhedral complexes). Let $X$ and $Y$ be (rational) polyhedral complexes. Then a morphism of polyhedral complexes is a map $\rho:|X| \rightarrow|Y|$ that satisfies, for each polyhedron $\sigma \in X$ :

(i) $\rho(\sigma) \in Y$,

(ii) $\left.\rho\right|_{\sigma}$ is affine linear,

(iii) $\rho\left(\Lambda_{\sigma}\right) \subseteq \Lambda_{\rho(\sigma)}$.

We call $\rho$ an isomorphism of polyhedral complexes if there exists an inverse morphism. In other words, an isomorphism is a bijection between $|X|$ and $|Y|$ (as well as between $X$ and $Y$ ) and $\rho\left(\Lambda_{\sigma}\right)=\Lambda_{\rho(\sigma)}$ for all $\sigma \in X$.

Lemma 4.3 (Intersections of $\Psi$-functions with the boundary). The facets of the fan $\varphi_{I \mid J} \cdot \psi_{1}^{a_{1}} \cdot \ldots \cdot \psi_{n}^{a_{n}} \cdot \mathcal{M}_{n}$ with positive weight are 
precisely the cones $\sigma$ in $\mathcal{M}_{n}$ with the following properties. Consider a curve in the interior of $\sigma$. Let $E(V) \in[n]$ be the set of leaves adjacent to a vertex $V$, and let $P(V)$ be the $\operatorname{val}(V)$-fold partition of $[n]$ obtained by removing $V$. Then the following hold:

(a) There exists one special vertex $V_{\text {spec }}$ whose partition $P\left(V_{\text {spec }}\right)$ is a subpartition of $I \mid J$ and whose valence is $\left(\sum_{k \in E(V)} a_{k}\right)+$ 4 .

(b) Let $m_{I}$ be the number of sets in $P\left(V_{\text {spec }}\right)$ contained in $I$. Then $m_{I}+1=\left(\sum_{k \in E(V) \cap I} a_{k}\right)+3$ (together with (a), the analogue $m_{J}+1=\left(\sum_{k \in E(V) \cap J} a_{k}\right)+3$ follows $)$. In particular, $m_{I}$, $m_{J}>1$.

(c) The valence of all other vertices $V$ equals $\left(\sum_{k \in E(V)} a_{k}\right)+3$.

Furthermore, the facets of $\varphi_{I \mid J} \cdot \psi_{1}^{a_{1}} \cdot \ldots \cdot \psi_{n}^{a_{n}} \cdot \mathcal{M}_{n}$ with negative weight fulfill the same properties (a) and (c) and the property:

$\left(\mathrm{b}^{\prime}\right)$ Let $m_{I}$ (respectively, $\left.m_{J}\right)$ be the number of sets in $P\left(V_{\mathrm{spec}}\right)$ contained in $I$ (respectively, $J)$. Then $m_{I}=1$ or $m_{J}=1$, i.e., $I \in P\left(V_{\text {spec }}\right)$ or $J \in P\left(V_{\text {spec }}\right)$.

Proof. We know how $X:=\psi_{1}^{a_{1}} \cdot \ldots \cdot \psi_{n}^{a_{n}} \cdot \mathcal{M}_{n}$ looks by Theorem 2.14 . In the combinatorial type of a facet of $X$ the valence of each vertex is $\left(\sum_{k \in E(V)} a_{k}\right)+3$; in the combinatorial type of a ridge, there is one special vertex $V_{\text {spec }}$ with valence $\left(\sum_{k \in E(V)} a_{k}\right)+4$. The balancing condition of a ridge is given by the equation:

$$
\sum_{I^{\prime} \mid J^{\prime}} \omega_{I^{\prime} \mid J^{\prime}} V_{I^{\prime} \mid J^{\prime}}=\sum_{\substack{I^{\prime} \mid J^{\prime} \\ I^{\prime} \in P\left(V_{\text {spec }}\right)}} \lambda_{I^{\prime} \mid J^{\prime}} V_{I^{\prime} \mid J^{\prime}}
$$

where the left hand sum runs through all superpartitions $I^{\prime} \mid J^{\prime}$ of $P\left(V_{\text {spec }}\right)$ not appearing in the right hand sum, $\omega_{I^{\prime} \mid J^{\prime}}$ denotes the weight of the facet obtained by inserting an edge $I^{\prime} \mid J^{\prime}$ and $\lambda_{I^{\prime} \mid J^{\prime}}$ is some (rational) coefficient. Therefore, the weight $\omega$ that this ridge obtains when intersecting $X$ with $\varphi_{I \mid J}$ is given by:

$$
\omega= \begin{cases}0 & \text { if } I \mid J \text { is not a superpartition of } P\left(V_{\text {spec }}\right), \\ \lambda_{I \mid J} & \text { if } I \in P\left(V_{\text {spec }}\right) \text { or } J \in P\left(V_{\text {spec }}\right), \\ \omega_{I \mid J} & \text { otherwise. }\end{cases}
$$


This already shows two implications. As all weights $\omega_{I^{\prime} \mid J^{\prime}}$ are at least non-negative, a ridge can only obtain a negative weight if it fulfills conditions (a), ( $\left.b^{\prime}\right)$ and (c). On the other hand, if a ridge of $X$ satisfies properties (a), (b) and (c), then $\omega_{I \mid J}$, and hence the ridge, obtains a positive weight. It remains to show the converse, which can be done by proving that all $\lambda_{I^{\prime} \mid J^{\prime}}$ are non-negative. To see this, we consider the balancing equation in $\mathbb{R}^{\left(\begin{array}{l}r \\ 2\end{array}\right)}$ and compare some coordinate entries.

Let $K$ be an arbitrary element of $P\left(V_{\text {spec }}\right)$; we want to show that $\lambda_{K}:=\lambda_{K \mid K^{c}}$ is non-negative. We choose two more arbitrary elements $L_{1}$ and $L_{2}$ in $P\left(V_{\text {spec }}\right)$ and fix some leaves $k \in K, l_{i} \in L_{i}$. Now the $k, l_{i}$-entry of the right hand side equals $\lambda_{K}+\lambda_{L_{i}}$ and analogously the $l_{1}, l_{2}$-entry equals $\lambda_{L_{1}}+\lambda_{L_{2}}$. Therefore, by adding the two $k, l_{i}$-entries and subtracting the $l_{1}, l_{2}$-entry, we get $2 \lambda_{K}$. Meanwhile, on the left hand side, we get

$$
2 \lambda_{K}=\sum_{\substack{I^{\prime} \mid J^{\prime} \\ k \in I^{\prime} \\ l_{1} \in J^{\prime}}} \omega_{I^{\prime} \mid J^{\prime}}+\sum_{\substack{I^{\prime} \mid J^{\prime} \\ k \in I^{\prime} \\ l_{2} \in J^{\prime}}} \omega_{I^{\prime} \mid J^{\prime}}-\sum_{\substack{I^{\prime} \mid J^{\prime} \\ l_{1} \in I^{\prime} \\ l_{2} \in J^{\prime}}} \omega_{I^{\prime} \mid J^{\prime}}=\sum_{I^{\prime} \mid J^{\prime}} \alpha_{I^{\prime} \mid J^{\prime}} \omega_{I^{\prime} \mid J^{\prime}}
$$

where

$$
\alpha_{I^{\prime} \mid J^{\prime}}= \begin{cases}2 & \text { if } k \in I^{\prime}, l_{1}, l_{2} \in J^{\prime} \\ 0 & \text { if } k, l_{1} \in I^{\prime}, l_{2} \in J^{\prime} \\ 0 & \text { if } k, l_{2} \in I^{\prime}, l_{1} \in J^{\prime} \\ 0 & \text { if } k, l_{1}, l_{2} \in I^{\prime}\end{cases}
$$

But, as all the weights $\omega_{I^{\prime} \mid J^{\prime}}$ are non-negative, it follows that $\lambda_{K}$ is non-negative.

Proposition 4.4. The map,

$$
\begin{aligned}
\rho_{I \mid J}:\left(\prod_{k \in I} \psi_{k}^{a_{k}} \cdot \mathcal{M}_{I \cup\{x\}}\right) \times( & \left.\prod_{k \in J} \psi_{k}^{a_{k}} \cdot \mathcal{M}_{J \cup\{y\}}\right) \\
& \longrightarrow\left(\varphi_{I \mid J} \cdot \psi_{1}^{a_{1}} \cdot \ldots \cdot \psi_{n}^{a_{n}} \cdot \mathcal{M}_{n}\right)^{+},
\end{aligned}
$$

is a well-defined isomorphism of polyhedral complexes.

Proof. We need to check the conditions of Definition 4.2. Using the lengths of the bounded edges as local coordinates on the cones, 
this follows directly from the description of the target complex in Lemma 4.3. The inverse map is given by splitting a given curve at its special vertex $V_{\text {spec }}$.

\subsection{The case of parametrized curves.}

Definition 4.5. Let $I \mid J$ be a reducible partition, and let $\Delta_{I}, \Delta_{J}$ be the corresponding splitting of the tropical degree $\Delta$. Let $Z=$ $\max \left(x_{1}, y_{1}\right) \cdot \ldots \cdot \max \left(x_{r}, y_{r}\right) \cdot \mathbb{R}^{r} \times \mathbb{R}^{r}$ denote the diagonal in $\mathbb{R}^{r} \times \mathbb{R}^{r}$, and consider the map:

$$
\mathrm{ev}_{x} \times \mathrm{ev}_{y}: \mathcal{M}_{I \cup\{x\}}^{\mathrm{lab}}\left(\mathbb{R}^{r}, \Delta_{I}\right) \times \mathcal{M}_{J \cup\{y\}}^{\mathrm{lab}}\left(\mathbb{R}^{r}, \Delta_{J}\right) \longrightarrow \mathbb{R}^{r} \times \mathbb{R}^{r} .
$$

We define

$$
Z_{I \mid J}:=\left(\mathrm{ev}_{x} \times \mathrm{ev}_{y}\right)^{*}(Z)
$$

We furthermore define $\pi_{I \mid J}: Z_{I \mid J} \rightarrow \mathcal{M}_{n}^{\text {lab }}\left(\mathbb{R}^{r}, \Delta\right)$ by

$$
\begin{aligned}
\mathcal{M}_{I \cup\{x\}} \times \mathbb{R}^{r} \times \mathcal{M}_{J \cup\{y\}} \times \mathbb{R}^{r} & \longrightarrow \mathcal{M}_{[n] \cup \Delta} \times \mathbb{R}^{r} \\
\left(\left(p_{I}, P\right),\left(p_{J}, Q\right)\right) & \longmapsto\left(\rho\left(p_{I}, p_{J}\right), P\right),
\end{aligned}
$$

where we choose the same anchor leaf for $\mathcal{M}_{I \cup\{x\}}^{\text {lab }}\left(\mathbb{R}^{r}, \Delta_{I}\right)$ and $\mathcal{M}_{n}^{\text {lab }}\left(\mathbb{R}^{r}, \Delta\right)$, and $\rho$ is the glueing map for abstract curves from the previous subsection.

Proposition 4.6. The map

$$
\pi_{I \mid J}: \psi_{1}^{a_{1}} \cdot \ldots \cdot \psi_{n}^{a_{n}} \cdot Z_{I \mid J} \longrightarrow\left(\varphi_{I \mid J} \cdot \psi_{1}^{a_{1}} \cdot \ldots \cdot \psi_{n}^{a_{n}} \cdot \mathcal{M}_{n}^{\mathrm{lab}}\left(\mathbb{R}^{r}, \Delta\right)\right)^{+}
$$

is a well-defined isomorphism of polyhedral complexes.

Proof. This follows from Proposition 4.4 and from $\left.\mathrm{ev}_{x}\right|_{Z_{I \mid J}}=$ $\left.\mathrm{ev}_{y}\right|_{Z_{I \mid J}}$ (which follows from both Proposition 1.11 ( $Z$ is described by convex functions) as well as from Proposition $1.12\left(\mathrm{ev}_{x} \times \mathrm{ev}_{y}\right.$ can be considered as a projection)).

Remark 4.7. Restricting to curves from $Z_{I \mid J}$ makes sure that the positions of the marked leaves are preserved under $\pi_{I \mid J}$, i.e., (by abuse of notation) for all $i \in I$, but also $j \in J$, we have $\mathrm{ev}_{i} \circ \pi_{I \mid J}=\mathrm{ev}_{i}$, respectively, $\mathrm{ev}_{j} \circ \pi_{I \mid J}=\mathrm{ev}_{j}$. 
Lemma 4.8. Let $E=\left(\varphi_{I \mid J} \cdot \tau_{a_{1}}\left(C_{1}\right) \cdot \ldots \cdot \tau_{a_{n}}\left(C_{n}\right)\right)_{\Delta}$ be a zerodimensional cycle. Then all points of $E$ lie in $\left(\varphi_{I \mid J} \cdot \psi_{1}^{a_{1}} \cdot \ldots \cdot \psi_{n}^{a_{n}}\right.$. $\left.\mathcal{M}_{n}^{\mathrm{lab}}\left(\mathbb{R}^{r}, \Delta\right)\right)^{+}$.

Proof. By Proposition 1.1, we can compute the weight of a point $p \in$ $E$ locally around $p$ in $X:=\varphi_{I \mid J} \cdot \psi_{1}^{a_{1}} \cdot \ldots \cdot \psi_{n}^{a_{n}} \cdot \mathcal{M}_{n}^{\text {lab }}\left(\mathbb{R}^{r}, \Delta\right)$, namely, we can focus on $\operatorname{Star}_{X}(p)$. Assume $p \notin\left(\varphi_{I \mid J} \cdot \psi_{1}^{a_{1}} \cdot \ldots \cdot \psi_{n}^{a_{n}} \cdot \mathcal{M}_{n}^{\text {lab }}\left(\mathbb{R}^{r}, \Delta\right)\right)^{+}$. Then curves corresponding to points in $\operatorname{Star}_{X}(p)$ contain a bounded edge corresponding to the partition $I \mid J$ (see equation (4.3)). But, as $I \mid J$ is chosen to be reducible, this edge is a contracted bounded edge whose length does not change the positions of the marked leaves in $\mathbb{R}^{r}$. Therefore, if we denote by ev $=\mathrm{ev}_{1} \times \ldots \times \mathrm{ev}_{n}$ the product of all evaluation maps, the image of $\operatorname{Star}_{X}(p)$ under ev has smaller dimension, which implies $\operatorname{ev}_{*}\left(\operatorname{Star}_{X}(p)\right)=0$. Hence, by projection formula, the weight of $p$ in $E$ must be zero.

The following statement combines Proposition 1.15, in particular item (iii), and the preceding result.

Corollary 4.9. Let $E=\left(\varphi_{I \mid J} \cdot \tau_{a_{1}}\left(C_{1}\right) \cdot \ldots \cdot \tau_{a_{n}}\left(C_{n}\right)\right)_{\Delta}$ be a zerodimensional cycle. If we substitute the cycles $C_{i}$ by general translations, we can assume that all points of $E$ lie in the interior of a facet of $\left(\varphi_{I \mid J} \cdot \psi_{1}^{a_{1}} \cdot \ldots \cdot \psi_{n}^{a_{n}} \cdot \mathcal{M}_{n}^{\mathrm{lab}}\left(\mathbb{R}^{r}, \Delta\right)\right)^{+}$. This operation does not change the degree of $E$ by Remark 1.13.

As a provisional result of this discussion, we can formulate the following:

Proposition 4.10. Let $E=\left(\varphi_{I \mid J} \cdot \tau_{a_{1}}\left(C_{1}\right) \cdot \ldots \cdot \tau_{a_{n}}\left(C_{n}\right)\right)_{\Delta}$ be a zerodimensional cycle. Then the equation,

$\left\langle\varphi_{I \mid J} \cdot \tau_{a_{1}}\left(C_{1}\right) \cdot \ldots \cdot \tau_{a_{n}}\left(C_{n}\right)\right\rangle_{\Delta}=\left\langle\tau_{a_{1}}\left(C_{1}\right) \cdot \ldots \cdot \tau_{a_{n}}\left(C_{n}\right) \cdot Z_{I \mid J}\right\rangle_{\Delta_{I}, \Delta_{J}}$, holds.

Proof. We denote $X:=\psi_{1}^{a_{1}} \ldots \cdot \psi_{n}^{a_{n}} \cdot Z_{I \mid J}$ and $Y:=\varphi_{I \mid J}$. $\psi_{1}^{a_{1}} \cdot \ldots \cdot \psi_{n}^{a_{n}} \cdot \mathcal{M}_{n}^{\mathrm{lab}}\left(\mathbb{R}^{r}, \Delta\right)$ and assume that the conditions $C_{i}$ are general. Then Corollary 4.9 implies that, for each point $p \in E$, we 
have an isomorphism of cycles $\pi_{I \mid J}: \operatorname{Star}_{X}\left(\pi_{I \mid J}^{-1}(p)\right) \rightarrow \operatorname{Star}_{Y}(p)$. By Proposition 1.1, this suffices to show that the weights of $p$ and $\pi_{I \mid J}^{-1}(p)$ in their respective intersection products coincide.

4.3. Splitting the diagonal. To this point, we have seen that intersecting with a "boundary" function $\varphi_{I \mid J}$ leads to intersection products in two smaller moduli spaces, $\mathcal{M}_{I \cup\{x\}}^{\mathrm{lab}}\left(\mathbb{R}^{r}, \Delta_{I}\right)$ and $\mathcal{M}_{J \cup\{y\}}^{\mathrm{lab}}\left(\mathbb{R}^{r}, \Delta_{J}\right)$. However, the factor $\left(\mathrm{ev}_{x} \times \mathrm{ev}_{y}\right)^{*}(Z)$ still connects these two smaller spaces. In order to obtain independent intersection products on the smaller spaces, we must split the diagonal contribution. In the algebrogeometric case, this can easily be done as the class of the diagonal $Z$, e.g., $\mathbb{P}^{r} \times \mathbb{P}^{r}$ can be written as the sum of products of classes in the factors

$$
[Z]=\left[L^{0} \times L^{r}\right]+\left[L^{1} \times L^{r-1}\right]+\cdots+\left[L^{r} \times L^{0}\right],
$$

where $L^{i}$ denotes an $i$-dimensional linear space in $\mathbb{P}^{r}$. But this can not be copied directly in our setting (see below). In some sense, for the first time, we meet a disadvantage due to the non-compactness of our spaces. Our notion of rational equivalence is "too strong" for this application, as it is inspired by the idea that two rationally equivalent objects should be rationally equivalent in any toric compactification. However, we will discuss here how far the conventional plan can be carried out anyway.

The general plan is the following. Set

$$
X_{I}:=\left(\tau_{0}\left(\mathbb{R}^{r}\right) \cdot \prod_{k \in I} \tau_{a_{k}}\left(C_{k}\right)\right)_{\Delta_{I}} \quad \text { in } \mathcal{M}_{I \cup\{x\}}^{\mathrm{lab}}\left(\mathbb{R}^{r}, \Delta_{I}\right)
$$

and

$$
X_{J}:=\left(\tau_{0}\left(\mathbb{R}^{r}\right) \cdot \prod_{k \in J} \tau_{a_{k}}\left(C_{k}\right)\right)_{\Delta_{J}} \quad \text { in } \mathcal{M}_{J \cup\{y\}}^{\mathrm{lab}}\left(\mathbb{R}^{r}, \Delta_{J}\right) .
$$

We want to compute the degree of

$$
\left(\tau_{a_{1}}\left(C_{1}\right) \cdot \ldots \cdot \tau_{a_{n}}\left(C_{n}\right) \cdot Z_{I \mid J}\right)_{\Delta_{I}, \Delta_{J}}=\left(\mathrm{ev}_{x} \times \mathrm{ev}_{y}\right)^{*}(Z) \cdot\left(X_{I} \times X_{J}\right),
$$

or, by the projection formula,

$$
\operatorname{deg}\left(Z \cdot\left(\operatorname{ev}_{x}\left(X_{I}\right) \times \operatorname{ev}_{y}\left(X_{J}\right)\right)\right) .
$$


Now we would like to replace the diagonal $Z$ by something like

$$
S:=\sum_{\alpha}\left(M_{\alpha} \times N_{\alpha}\right),
$$

where $M_{\alpha}$ and $N_{\alpha}$ are cycles in $\mathbb{R}^{r}$ such that $S$ intersects $e_{x}\left(X_{I}\right) \times$ $\operatorname{ev}_{y}\left(X_{J}\right)$ like $Z$. But note that $S$ cannot be rationally equivalent to $Z$ (in the sense of [2]), as this would imply that both cycles must have the same recession fan, i.e., the same directions towards infinity. To come out of this, we need more information about what the push-forwards $\mathrm{ev}_{x}\left(X_{I}\right)$ and $\mathrm{ev}_{y}\left(X_{J}\right)$ look like; in particular, we would like to know how their degrees/recession fans can look. Let us formalize this first.

Let $\Theta$ be a complete simplicial fan in $\mathbb{R}^{r}$, and let $Z_{k}(\Theta)$ be the group of $k$-dimensional cycles $X$ whose support lies in the $k$-dimensional skeleton of $\Theta$, i.e., $|X| \subseteq\left|\Theta^{(k)}\right|$. Fix a basis of $Z_{*}(\Theta):=\oplus_{k=0}^{r} Z_{k}(\Theta)$ denoted by $B_{0}, \ldots, B_{m}$ (where we may assume $B_{0}=\{0\}$ and $B_{m}=$ $\mathbb{R}^{r}$ ). If the degree $\delta(X)$ of an arbitrary cycle is contained in $Z_{*}(\Theta)$, we say $X$ is $\Theta$-directional. For such a cycle, there exist integer coefficients $\lambda_{e}$ such that $X \sim \delta(X)=\sum_{e=1}^{m} \lambda_{e} B_{e}$. For each ray $\rho \in \Theta^{(1)}$ with primitive vector $v_{\rho}$, let $\varphi_{\rho}$ be the rational function on $\Theta$ uniquely defined by

$$
\varphi_{\rho}\left(v_{\rho^{\prime}}\right)= \begin{cases}1 & \text { if } \rho^{\prime}=\rho \\ 0 & \text { otherwise }\end{cases}
$$

Lemma 4.11. The linear map

$$
\begin{aligned}
Z_{*}(\Theta) & \longrightarrow \mathbb{Z}^{m+1}, \\
X & \longmapsto\left(\operatorname{deg}\left(B_{0} \cdot X\right), \ldots, \operatorname{deg}\left(B_{m} \cdot X\right)\right),
\end{aligned}
$$

(where $\operatorname{deg}(\cdot)$ is set to be zero if the dimension of the argument is non-zero) is injective.

Proof. Let $X \in Z_{k}(\Theta)$ be an element of the kernel, which implies that $\operatorname{deg}(X \cdot Y)=0$ for all $Y \in Z_{r-k}(\Theta)$. Now, in fact, the remainder is identical to the proof of [2, Lemma 6]. Assume that $X$ is non-zero and therefore there exists a cone $\sigma \in \Theta^{(k)}$ such that $\omega_{X}(\sigma) \neq 0$. As $\Theta$ is simplicial, this cone is generated by $k$ rays, $\rho_{1}, \ldots, \rho_{k}$. Let us consider $\varphi_{\rho_{k}} \cdot X$ and, in particular, the weight of $\tau:=\left\langle\rho_{1}, \ldots, \rho_{k-1}\right\rangle$ in this intersection product. As primitive vector $v_{\sigma / \tau}$, we can use 
$1 /\left|\Lambda_{\sigma} /\left(\Lambda_{\tau}+\Lambda_{\rho_{k}}\right)\right| v_{\rho_{k}}$ (it might not be an integer vector, but modulo $V_{\tau}$, it is a primitive generator of $\left.\sigma\right)$. Analogously, we can get any primitive vector around $\tau$ as a multiple of an appropriate $v_{\rho}$. But, as $\varphi_{\rho_{k}}$ is zero on all of these vectors except $v_{\rho_{k}}$, we get

$$
\omega_{\varphi_{\rho_{k}} \cdot X}(\tau)=\frac{\omega_{X}(\sigma)}{\left|\Lambda_{\sigma} /\left(\Lambda_{\tau}+\Lambda_{\rho_{k}}\right)\right|} \neq 0 .
$$

Now, induction shows

$$
\begin{aligned}
\operatorname{deg}\left(\varphi_{\rho_{1}} \cdots \varphi_{\rho_{k}} \cdot X\right) & =\omega_{\varphi_{\rho_{1}} \cdots \varphi_{\rho_{k}} \cdot X}(\{0\}) \\
& =\frac{\omega_{X}(\sigma)}{\left|\Lambda_{\sigma} /\left(\Lambda_{\rho_{1}}+\cdots+\Lambda_{\rho_{k}}\right)\right|} \neq 0 .
\end{aligned}
$$

This means we have found a $\Theta$-directional cycle $Y:=\varphi_{\rho_{1}} \cdots \varphi_{\rho_{k}} \cdot \mathbb{R}^{r} \in$ $Z_{r-k}(\Theta)$ with $\operatorname{deg}(X \cdot Y) \neq 0$, which contradicts the assumption that $X$ is an element of the kernel.

With respect to the basis $B_{0}, \ldots, B_{m}$, the map defined in the previous lemma has the matrix representation $\alpha:=\left(\operatorname{deg}\left(B_{e} \cdot B_{f}\right)\right)_{e f}$. Obviously, $\alpha$ is a symmetric matrix. The lemma implies that this matrix is invertible over $\mathbb{Q}$, and we denote the inverse by $\left(\beta_{e f}\right)_{e f}$. The coefficients of this matrix can be used to replace the diagonal $Z$ of $\mathbb{R}^{r} \times \mathbb{R}^{r}$ by a sum of products of cycles in the two factors (namely $\left.\sum_{e, f} \beta_{e f}\left(B_{e} \times B_{f}\right)\right)$, at least with respect to $\Theta$-directional cycles.

Lemma 4.12. Let $X \sim \sum_{e} \lambda_{e} B_{e}$ and $Y \sim \sum_{f} \mu_{e} B_{e}$ be two $\Theta$ directional cycles in $\mathbb{R}^{r}$ with complementary dimension. Then

$$
\begin{aligned}
\operatorname{deg}(Z \cdot(X \times Y)) & =\operatorname{deg}(X \cdot Y) \\
& =\sum_{e, f} \operatorname{deg}\left(X \cdot B_{e}\right) \beta_{\text {ef }} \operatorname{deg}\left(Y \cdot B_{f}\right) .
\end{aligned}
$$

Proof. Denote $\lambda:=\left(\lambda_{1}, \ldots, \lambda_{m}\right), \mu:=\left(\mu_{1}, \ldots, \mu_{m}\right)$. We get

$$
\begin{aligned}
\sum_{e, f} & \operatorname{deg}\left(X \cdot B_{e}\right) \beta_{e f} \operatorname{deg}\left(Y \cdot B_{f}\right) \\
& =(\alpha \cdot \lambda)^{T} \cdot \beta \cdot(\alpha \cdot \mu)=\lambda^{T} \cdot \alpha^{T} \cdot \beta \cdot \alpha \cdot \mu=\lambda^{T} \cdot \alpha \cdot \beta \cdot \alpha \cdot \mu \\
& =\lambda^{T} \cdot \alpha \cdot \mu=\operatorname{deg}(X \cdot Y) .
\end{aligned}
$$


Using this, our original goal of deriving a tropical splitting lemma can be formulated as follows.

Theorem 4.13 (Splitting lemma). Let $E=\left(\varphi_{I \mid J} \cdot \prod_{k=1}^{n} \tau_{a_{k}}\left(C_{k}\right)\right)_{\Delta}^{\mathbb{R}^{r}}$ be a zero-dimensional cycle, where $I \mid J$ is a reducible partition. Moreover, let us assume that $\Theta$ is a complete simplicial fan such that (with the notation from above) $\mathrm{ev}_{x}\left(X_{I}\right)$ and $\mathrm{ev}_{y}\left(X_{J}\right)$ are $\Theta$-directional. Let $B_{0}, \ldots, B_{m}$ be a basis of $Z_{*}(\Theta)$, and let $\left(\beta_{\text {ef }}\right)_{\text {ef }}$ be the inverse matrix $($ over $\mathbb{Q})$ of $\left(\operatorname{deg}\left(B_{e} \cdot B_{f}\right)\right)_{\text {ef }}$. Then the following equation holds:

$$
\begin{aligned}
& \left\langle\varphi_{I \mid J} \cdot \prod_{k=1}^{n} \tau_{a_{k}}\left(C_{k}\right)\right\rangle_{\Delta} \\
& \quad=\sum_{e, f}\left\langle\prod_{k \in I} \tau_{a_{k}}\left(C_{k}\right) \cdot \tau_{0}\left(B_{e}\right)\right\rangle_{\Delta_{I}} \beta_{e f}\left\langle\tau_{0}\left(B_{f}\right) \cdot \prod_{k \in J} \tau_{a_{k}}\left(C_{k}\right)\right\rangle_{\Delta_{J}} .
\end{aligned}
$$

Proof. The proof follows from the general plan above and Proposition 4.10 .

Remark 4.14. Of course, in toric geometry language, the basis $B_{0}, \ldots, B_{m}$ corresponds to a basis $\gamma_{0}, \ldots, \gamma_{m}$ of the cohomology groups of $\mathbf{X}(\Theta)$ (the toric variety associated to $\Theta$ ). As the cup-product and the intersection product of cycles are equivalent (cf., Theorem 1.9), the corresponding matrix $\left(\operatorname{deg}\left(\gamma_{e} \cup \gamma_{f}\right)\right)_{e f}$ is equal to $\alpha$. This implies that the coefficients $\beta_{\text {ef }}$ appearing in the tropical splitting lemma really are the same as in the associated algebro-geometric version.

4.4. The directions of families of curves. The above splitting lemma is only useful if, at least for a certain class of invariants, the fan of directions $\Theta$ is fixed and well known. This is one of the main problems when transferring the algebro-geometric theory to the tropical set-up. However, in this subsection, we will show that in some cases the problem can be solved.

Remark 4.15. In the easiest case, namely if $r=1$, the situation is trivial. There is one unique complete simplicial fan $\Theta=$ 
$\left\{\mathbb{R}_{\leq 0},\{0\}, \mathbb{R}_{\geq 0}\right\}$, and any subcycle is $\Theta$-directional. Also, with $B_{0}=$ $\{0\}, B_{1}=\mathbb{R}$, the statement of Lemma 4.12 is obvious here.

Let us now consider curves in the plane, i.e., $r=2$. Let

$$
F=\left(\tau_{0}\left(\mathbb{R}^{2}\right) \cdot \prod_{k=1}^{n} \tau_{a_{k}}\left(C_{k}\right)\right)_{\Delta}^{\mathbb{R}^{2}}
$$

be a one-dimensional family of plane curves (with unrestricted leaf $x_{0}$ ). We define $\Theta(F)$ to be the complete fan in $\mathbb{R}^{2}$ which contains the following rays: all directions appearing in $\Delta$ and, furthermore, all rays in $\delta\left(C_{k}\right)$ if $\operatorname{dim}\left(C_{k}\right)=1$ and $a_{k}>0$.

Proposition 4.16. Let $F=\left(\tau_{0}\left(\mathbb{R}^{2}\right) \cdot \prod_{k=1}^{n} \tau_{a_{k}}\left(C_{k}\right)\right)_{\Delta}^{\mathbb{R}^{2}}$ be a onedimensional family of plane curves (with unrestricted leaf $\left.x_{0}\right)$. Let us furthermore assume that $a_{k} \leq 1$ if $\operatorname{dim}\left(C_{k}\right)=2$ (i.e., if a leaf is not restricted by ev-conditions, only one $\Psi$-condition is allowed). Then $\mathrm{ev}_{0 *}(F)$ is $\Theta(F)$-directional.

Proof. As before, we replace each factor $\psi_{k}^{a_{k}}$ by $\mathrm{ft}_{0}^{*}\left(\psi_{k}\right)^{a_{k}}+$ $\mathrm{ft}_{0}^{*}\left(\psi_{k}\right)^{a_{k}-1} \cdot \varphi_{0, k}$ and multiply out. Consider the term without $\varphi$ factors. It is the fibre of $\left(\prod_{k=1}^{n} \tau_{a_{k}}\left(C_{k}\right)\right)_{\Delta}$ (which is finite) under $\mathrm{ft}_{0}$ (see family property (3.8)) and, moreover, the push-forward of the fibre along $\mathrm{ev}_{0}$ is just the sum/union of the images in $\mathbb{R}^{r}$ of the parametrized curves corresponding to the points in $\left(\prod_{k=1}^{n} \tau_{a_{k}}\left(C_{k}\right)\right)_{\Delta}$. But these curves have degree $\Delta$; thus, by definition, their images are $\Theta(F)$-directional.

So let us consider the term with the factor $\varphi_{0, k}$. Here, $\mathrm{ev}_{0}$ and $\mathrm{ev}_{k}$ coincide (see Lemma 3.14), so we can in fact compute the pushforward along $\mathrm{ev}_{k}$. As $\mathrm{ev}_{k}=\mathrm{ev}_{k} \circ \mathrm{ft}_{0}$ (by abuse of notation), we can first push-forward along $\mathrm{ft}_{0}$ and get the term $\left(\tau_{a_{k}-1}\left(C_{k}\right) \cdot \prod_{l \neq k} \tau_{a_{l}}\left(C_{l}\right)\right)$.

Now, if $\operatorname{dim}\left(C_{k}\right)=2$, by our assumptions $a_{k}-1=0$, in which case we can use induction to prove the statement, or this term does not appear at all.

On the other hand, if $\operatorname{dim}\left(C_{k}\right)=0,1$, we can use the fact that the push-forward is certainly contained in $C_{k}$; therefore, $\operatorname{dim}\left(C_{k}\right)=0$ is 
trivial and $\operatorname{dim}\left(C_{k}\right)=1$ works as we added the directions of $C_{k}$ to $\Theta(F)$ if $a_{k}>0$.

This finishes the proof, as all terms with more $\varphi$-factors vanish.

Remark 4.17. A weaker version of this lemma can be obtained by assuming general conditions and directly studying the behavior of $\mathrm{ev}_{0}$ on an unbounded ray in $F$ (see [17, Lemma 3.7]).

Remark 4.18. Consider the family $F=\left(\tau_{0}\left(\mathbb{R}^{2}\right) \tau_{0}(P) \tau_{2}\left(\mathbb{R}^{2}\right)\right)_{1}^{\mathbb{R}^{2}}=$ $\mathrm{ev}_{1}^{*}(P) \cdot \psi_{2}^{2} \cdot \mathcal{M}_{3}^{\mathrm{lab}}\left(\mathbb{R}^{2}, 1\right)$ of curves of projective degree 1 . It consists of the following types of curves.

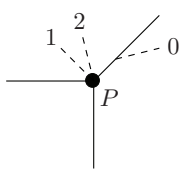

$\downarrow \mathrm{ev}_{0}$

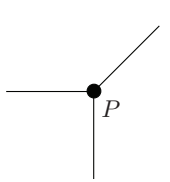

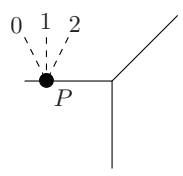

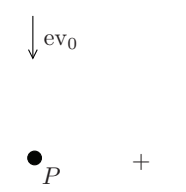

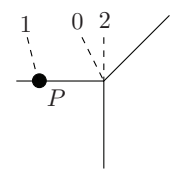
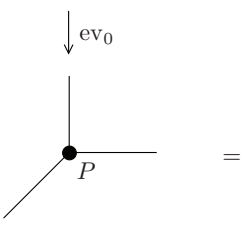

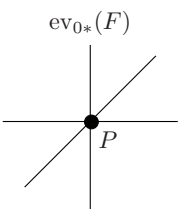

Its push-forward along ev $v_{0}$ also contains the inverted standard directions $(1,0),(0,1)$ and $(-1,-1)$. Therefore, this family is a counterexample to our statement if we drop the condition on the number of $\Psi$-conditions allowed at leaves not restricted by incidence conditions.

Remark 4.19. For higher dimensions $(r>2)$, only a few cases are explored. If we restrict to projective degree $d$ and banish all $\Psi$ conditions, i.e., for a family $F=\left(\tau_{0}\left(\mathbb{R}^{r}\right) \cdot \prod_{k=1}^{n} \tau_{0}\left(C_{k}\right)\right)_{d}$ of arbitrary dimension, it is proven in [10] that $\operatorname{ev}_{0 *}(F)$ is $\Theta$-directional, where $\Theta$ is the complete simplicial fan in $\mathbb{R}^{r}$ consisting of all cones generated by at most $r$ of the vectors $-e_{0},-e_{1}, \ldots,-e_{r}$. We conjecture that a similar proof also works for $\Psi$-conditions at point-conditions. 
5. WDVV equations and topological recursion. We are now ready to prove the tropical analogues of the WDVV and topological recursion equations under certain assumptions. With the help of these equations, we show that certain tropical gravitational descendants coincide with their classical counterparts. This reduces the computation of the classical invariants to a counting problem for tropical curves with certain valence and incidence conditions (cf., Remark 3.10).

5.1. WDVV equations. Let $x_{i}, x_{j}, x_{k}$ and $x_{l}$ be pairwise different marked leaves, and consider the forgetful map ft $: \mathcal{M}_{n}^{\text {lab }}\left(\mathbb{R}^{r}, \Delta\right) \rightarrow$ $\mathcal{M}_{\{i, j, k, l\}}$.

Lemma 5.1. The equation,

$$
\mathrm{ft}^{*}\left(\varphi_{\{i, j\} \mid\{k, l\}}\right)=\sum_{\substack{I \mid J \\ i, j \in I \\ k, l \in J}} \varphi_{I \mid J},
$$

holds, where the sum on the right side runs through all (also nonreducible) partitions with $i, j \in I$ and $k, l \in J$.

Proof. Note that

$$
\mathrm{ft}\left(V_{I \mid J}\right)=V_{I \cap\{i, j, k, l\} \mid J \cap\{i, j, k, l\}} .
$$

Therefore, $\varphi\left(\mathrm{ft}\left(V_{I \mid J}\right)\right)=1$ if $i, j \in I, k, l \in J$ and 0 otherwise.

Now we face the crucial difference to the conventional setting. The right sum also runs over non-reducible partitions, which do not correspond to anything in the algebro-geometric case. Let us add up only those $\varphi_{I \mid J}$ with $I \mid J$ non-reducible and denote the sum by $\phi$, i.e.,

$$
\phi_{i, j \mid k, l}:=\sum_{\substack{I \mid J \\ \text { non-red } \\ i, j \in I \\ k, l \in J}} \varphi_{I \mid J} .
$$

We would like to show that $\phi_{i, j \mid k, l}$ is bounded, as then it does not change the degree of an intersection product and we can derive the same formulas as in the conventional case. So let us investigate what this function measures. 
Let $F=\left(\prod_{k=1}^{n} \tau_{a_{k}}\left(C_{k}\right)\right)_{\Delta}$ be a one-dimensional family of curves with general conditions. Consider a facet $\sigma$ of $F$ representing curves with contracted bounded edge $E$ (called reducible curves). Then we can change the length of $E$ while keeping all other lengths and our curve will still match the incidence conditions. As our conditions are general, the set of curves fulfilling the incidence conditions settheoretically is also one-dimensional. Hence, all curves in $\sigma$ just differ by the length of $E$, whereas all other lengths are fixed. But this means that $\phi_{i, j \mid k, l}$ is constant on $\sigma$.

Now, let $\sigma$ be a facet of $F$ representing curves without contracted bounded edge $E$ (called non-reducible curves). This means, for all nonreducible partitions $I \mid J$, the respective function $\varphi_{I \mid J}$ is identically zero on $\sigma$. Therefore, on $\sigma, \phi_{i, j \mid k, l}$ coincides with $\mathrm{ft}^{*}\left(\varphi_{\{i, j\} \mid\{k, l\}}\right)$.

Lemma 5.2. Let

$$
F=\left(\prod_{k=1}^{n} \tau_{a_{k}}\left(C_{k}\right)\right)_{\Delta}
$$

be a one-dimensional family of curves with general conditions. Let $\sigma$ be a facet of $F$. Then

$\left.\phi_{i, j \mid k, l}\right|_{\sigma}= \begin{cases}\varphi_{\{i, j\} \mid\{k, l\}} \circ \mathrm{ft} & \text { if interior curves of } \sigma \text { are non-reducible, } \\ \text { const } & \text { otherwise. }\end{cases}$

In other words, proving that $\phi_{i, j \mid k, l}$ is bounded on a one-dimensional family $F$ is the same as proving that curves in $F$ with large $\mathcal{M}_{i, j, k, l^{-}}$ coordinate must contain a contracted bounded edge. This is the manner of speaking in existing literature (e.g., [9, Proposition 5.1], [14, Proposition 6.1], [17, Section 4]). We will address this problem in its own subsection and first state the desired results here.

Lemma 5.3. Let $F=\left(\prod_{k=1}^{n} \tau_{a_{k}}\left(C_{k}\right)\right)_{\Delta}$ be a one-dimensional family of curves. Furthermore, assume that $\phi_{i, j \mid k, l}$ is bounded. Then the equation 


$$
\left\langle\mathrm{ft}^{*}\left(\varphi_{\{i, j\} \mid\{k, l\}}\right) \cdot \prod_{k=1}^{n} \tau_{a_{k}}\left(C_{k}\right)\right\rangle_{\Delta}=\sum_{I \mid J} \sum_{\substack{\text { reducible } \\ k, l \in J}}\left\langle\varphi_{I \mid J} \cdot \prod_{k=1}^{n} \tau_{a_{k}}\left(C_{k}\right)\right\rangle_{\Delta}
$$

holds.

Proof. This follows from Lemma 5.1 and the fact that the degree of a bounded function intersected with a one-dimensional cycle is zero. Therefore, if $\phi_{i, j \mid k, l}$ is bounded, the degree of

$$
\left\langle\phi_{i, j \mid k, l} \cdot \prod_{k=1}^{n} \tau_{a_{k}}\left(C_{k}\right)\right\rangle_{\Delta}
$$

is zero, and hence this term can be omitted.

Finally, we can state the following version of WDVV equations. A more restrictive version was proven in [17, Theorem 8.1]. Let us emphasize again the difference of the two approaches. In [17], similar to previous works such as [9], the proof of certain WDVV equations was based on two steps. First, under generic conditions, it is shown explicitly that the curves under consideration split into two parts. Second, it is shown that the multiplicity of the big curve factors as a product of the two smaller parts. This is done by an involved computation in terms of a suitable matrix representation of $\mathrm{ev}_{1} \times \cdots \mathrm{ev}_{n} \times \mathrm{ft}(\mathrm{cf}$., $[\mathbf{1 7}$, Lemma 6.6]). In the present approach, these ad hoc computations are replaced by intersection-theoretic arguments (e.g., the splitting lemma).

As before, we fix a complete simplicial fan $\Theta$ and a basis $B_{0}, \ldots, B_{m}$ of $Z_{*}(\Theta)$. Furthermore, let $\left(\beta_{\text {ef }}\right)_{e f}$ be the inverse matrix (over $\mathbb{Q}$ ) of the matrix $\left(\operatorname{deg}\left(B_{e} \cdot B_{f}\right)\right)_{e f}$.

Theorem 5.4 (WDVV equations). Let $F=\left(\prod_{k=1}^{n} \tau_{a_{k}}\left(C_{k}\right)\right)_{\Delta}$ be a one-dimensional family of curves, and fix four pairwise different marked leaves $x_{i}, x_{j}, x_{k}, x_{l}$. Moreover, we assume that the following conditions hold. 
(i) For any reducible partition $I \mid J$ with $i, j \in I ; k, l \in J$ or $i, k \in I$; $j, l \in J$ the push-forwards $\mathrm{ev}_{x}\left(X_{I}\right)$ and $\mathrm{ev}_{y}\left(X_{J}\right)$ are $\Theta$-directional (with notations from Section 4 ).

(ii) The functions $\phi_{i, j \mid k, l}$ and $\phi_{i, k \mid j, l}$ are bounded on $F$.

Then the WDVV equation

$$
\begin{aligned}
& \sum_{\substack { I \mid J \\
\begin{subarray}{c}{\text { reducible } \\
i, j \in I \\
k, l \in J{ I | J \\
\begin{subarray} { c } { \text { reducible } \\
i , j \in I \\
k , l \in J } }\end{subarray}} \sum_{e, f}\left\langle\prod_{k \in I} \tau_{a_{k}}\left(C_{k}\right) \cdot \tau_{0}\left(B_{e}\right)\right\rangle_{\Delta_{I}} \beta_{e f}\left\langle\tau_{0}\left(B_{f}\right) \cdot \prod_{k \in J} \tau_{a_{k}}\left(C_{k}\right)\right\rangle_{\Delta_{J}} \\
= & \sum_{\substack {|| J \\
\begin{subarray}{c}{\text { reducible } \\
i, k \in I \\
j, l \in J{ | | J \\
\begin{subarray} { c } { \text { reducible } \\
i , k \in I \\
j , l \in J } }\end{subarray}} \sum_{e, f}\left\langle\prod_{k \in I} \tau_{a_{k}}\left(C_{k}\right) \cdot \tau_{0}\left(B_{e}\right)\right\rangle_{\Delta_{I}} \beta_{e f}\left\langle\tau_{0}\left(B_{f}\right) \cdot \prod_{k \in J} \tau_{a_{k}}\left(C_{k}\right)\right\rangle_{\Delta_{J}}
\end{aligned}
$$

holds, where the sums run through reducible partitions only.

Proof. The statement follows from Lemma 5.3 and the fact that on $\mathcal{M}_{\{i, j, k, l\}}$ the functions $\varphi_{\{i, j\} \mid\{k, l\}}$ and $\varphi_{\{i, k\} \mid\{j, l\}}$ are rationally equivalent. In fact, they only differ by a linear function and therefore have the same divisor, namely, the single vertex in $\mathcal{M}_{\{i, j, k, l\}}$.

Remark 5.5. In the algebro-geometric version of these equations (cf., $\left[6\right.$, equations (54), (55)]) the big sum(s) usually run like $\sum_{\beta_{1}, \beta_{2}} \sum_{A, B}$, where $\beta_{1}, \beta_{2}$ are cohomology classes such that $\beta_{1}+\beta_{2}=\beta$ and $A \cup B=[n]$ is a partition of the marks. We can proceed accordingly and let our sum run through unlabeled instead of labeled degrees, as unlabeled degrees correspond via Minkowski weights to cohomology classes. If we collect all reducible partitions $I \cup J=\Delta \cup[n]$, such that the unlabeled degrees $\delta\left(\Delta_{I}\right), \delta\left(\Delta_{J}\right)$ coincide, we obtain a class of $\Delta ! / \Delta_{I} ! \cdot \Delta_{J}$ ! elements. On the other hand, as mentioned at the beginning of Section 3, counting curves with labeled non-contracted leaves leads to an overcounting by the factor $\Delta$ !, i.e., if $\delta:=\delta(\Delta)$ is an unlabeled degree, we should define

$$
\left\langle\prod_{k=1}^{n} \tau_{a_{k}}\left(C_{k}\right)\right\rangle_{\delta}:=\frac{1}{\Delta !}\left\langle\prod_{k=1}^{n} \tau_{a_{k}}\left(C_{k}\right)\right\rangle_{\Delta} .
$$


So, by switching to "unlabeled" invariants, the above factor $\Delta ! / \Delta_{I} ! \cdot \Delta_{J}$ ! cancels, and we obtain

$$
\begin{aligned}
& \sum_{\substack{\delta_{I}, \delta_{J} \\
\delta_{I}+\delta_{J}=\delta}} \sum_{\substack{\cup \cup B=[n] \\
i, j \in A \\
k, l \in B}} \sum_{e, f} \\
& \left\langle\prod_{k \in A} \tau_{a_{k}}\left(C_{k}\right) \cdot \tau_{0}\left(B_{e}\right)\right\rangle_{\delta_{I}} \beta_{e f}\left\langle\tau_{0}\left(B_{f}\right) \cdot \prod_{k \in B} \tau_{a_{k}}\left(C_{k}\right)\right\rangle_{\delta_{J}} \\
& =\sum_{\substack{\delta_{I}, \delta_{J} \\
\delta_{I}+\delta_{J}=\delta}} \sum_{\substack{i \in B=[n] \\
i, k \in A \\
j, l \in B}} \sum_{e, f} \\
& \left\langle\prod_{k \in A} \tau_{a_{k}}\left(C_{k}\right) \cdot \tau_{0}\left(B_{e}\right)\right\rangle_{\delta_{I}} \beta_{e f}\left\langle\tau_{0}\left(B_{f}\right) \cdot \prod_{k \in B} \tau_{a_{k}}\left(C_{k}\right)\right\rangle_{\delta_{J}},
\end{aligned}
$$

which is now combinatorially identical to the algebro-geometric version.

5.2. Topological recursion. In the same flavor as in the previous subsection, we will also formulate a tropical version of the equations known as "topological recursion."

Let $x_{i}, x_{k}$ and $x_{l}$ be pairwise different marked leaves. We know from Lemma 2.24 that we can express the $\Psi$-divisor $\psi_{i}$ in terms of "boundary" divisors, namely,

$$
\operatorname{div}\left(\psi_{i}\right)=\sum_{\substack{I \mid J \\ i \in I \\ k, l \in J}} \operatorname{div}\left(\varphi_{I \mid J}\right) .
$$

Now, again, we give a name to the term that has no algebro-geometric counterpart:

$$
\phi_{i \mid k, l}=\sum_{I \mid J} \varphi_{\substack{i \in I \\ \text { non-red } \\ k, l \in J}} .
$$

As in the previous subsection, we can describe this function as follows. 
Lemma 5.6. Let $F=\left(\prod_{k=1}^{n} \tau_{a_{k}}\left(C_{k}\right)\right)_{\Delta}$ be a one-dimensional family of curves with general conditions. Let $\sigma$ be a facet of $F$. Then the restriction $\left.\phi_{i \mid k, l}\right|_{\sigma}$
"equals the sum of the lengths of the edges that sep- arate $i$ from $k, l$ if interior curves of $\sigma$ are non- reducible, and is constant otherwise."

Again, we fix a complete simplicial fan $\Theta$ and a basis $B_{0}, \ldots, B_{m}$ of $Z_{*}(\Theta)$. Furthermore, let $\left(\beta_{\text {ef }}\right)_{e f}$ be the inverse matrix (over $\mathbb{Q}$ ) of the matrix $\left(\operatorname{deg}\left(B_{e} \cdot B_{f}\right)\right)_{e f}$.

Theorem 5.7 (Topological recursion). Let

$$
F=\left(\prod_{k=1}^{n} \tau_{a_{k}}\left(C_{k}\right)\right)_{\Delta}
$$

be a one-dimensional family of curves, and fix three pairwise different marked leaves $x_{i}, x_{k}$ and $x_{l}$. Moreover, we assume that the following conditions hold:

(i) For any reducible partition $I \mid J$ with $i \in I, k, l \in J$, the pushforwards $\mathrm{ev}_{x}\left(X_{I}\right)$ and $\mathrm{ev}_{y}\left(X_{J}\right)$ are $\Theta$-directional (with notation from Section 4).

(ii) The function $\phi_{i \mid k, l}$ is bounded on $F$.

Then the topological recursion

$$
\begin{aligned}
& \left\langle\psi_{i} \cdot \prod_{k=1}^{n} \tau_{a_{k}}\left(C_{k}\right)\right\rangle_{\Delta} \\
= & \sum_{\substack{I \mid J \\
\text { reducible } \\
i \in I \\
k, l \in J}} \sum_{e, f}\left\langle\prod_{k \in I} \tau_{a_{k}}\left(C_{k}\right) \cdot \tau_{0}\left(B_{e}\right)\right\rangle_{\Delta_{I}} \beta_{e f}\left\langle\tau_{0}\left(B_{f}\right) \cdot \prod_{k \in J} \tau_{a_{k}}\left(C_{k}\right)\right\rangle_{\Delta_{J}}
\end{aligned}
$$

holds, where the sum runs through reducible partitions only. 
Remark 5.8. In the same way as in Remark 5.5, we obtain the "unlabeled" version

$$
\begin{aligned}
\left\langle\psi_{i} \cdot \prod_{k=1}^{n} \tau_{a_{k}}\left(C_{k}\right)\right\rangle_{\delta}= & \sum_{\substack{\delta_{I}, \delta_{J} \\
\delta_{I}+\delta_{J}=\delta}} \sum_{\substack{i \in A \\
i \in A \\
k, l \in B}} \\
& \sum_{e, f}\left\langle\prod_{k \in A} \tau_{a_{k}}\left(C_{k}\right) \cdot \tau_{0}\left(B_{e}\right)\right\rangle_{\delta_{I}} \beta_{e f}\left\langle\tau_{0}\left(B_{f}\right) \cdot \prod_{k \in B} \tau_{a_{k}}\left(C_{k}\right)\right\rangle_{\delta_{J}},
\end{aligned}
$$

which coincides combinatorially with the algebro-geometric version of this equation.

5.3. Contracted bounded edges. As preparation for the more difficult case of plane curves, we first assume $r=1$.

Proposition 5.9. Let $P_{1}, \ldots, P_{n}$ be points in the general position in $\mathbb{R}^{1}$, and let

$$
F=\left(\prod_{k=1}^{n} \tau_{a_{k}}\left(P_{k}\right)\right)_{d}^{\mathbb{R}^{1}}
$$

be a one-dimensional family in $\mathcal{M}_{n}^{\mathrm{lab}}\left(\mathbb{R}^{1}, d\right)$. Then, for any choice of marked leaves $x_{i}, x_{j}, x_{k}$ and $x_{l}$, the functions $\phi_{i, j \mid k, l}$ and $\phi_{i \mid k, l}$ are bounded on $F$.

Proof. For general conditions, $F$ set-theoretically coincides with the set of curves satisfying the given incidence and valence conditions. Consider a general curve $C \in F$. Then $C$ is also a general curve in the $\Psi$-product $X:=\prod_{k=1}^{n} \psi_{k}^{a_{k}}$. As we cut down $X$ by $n$ point conditions and $\operatorname{dim}(F)=1$, the dimension of $X$ must be $n+1$; hence, $C$ contains $n$ bounded edges. This implies that $C$, as a rational curve, has $n+1$ vertices. Therefore, there exists a vertex $V$ not adjacent to a marked leaf $x_{k}, k \in[n]$. Now one of the three adjacent edges might be a contracted bounded edge. Then, the deformation of $C$ in $F$ is given by changing the length of this edge, but this does not affect $\phi_{i, j \mid k, l}$ or $\phi_{i \mid k, l}$ by definition. Otherwise, if all of the adjacent edges 


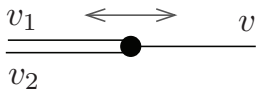

are non-contracted, the deformation of $C$ in $F$ is given by moving $V$ (and changing the lengths accordingly).

Note that the edge $v$ cannot be unbounded as its direction "vector" is not primitive. Therefore, if this deformation is supposed to be unbounded, $v_{1}$ and $v_{2}$ must be unbounded. But, in this case, only the length of $v$ grows infinitely. But as $v$ does not separate any marked leaves, this does not change $\phi_{i, j \mid k, l}$ and $\phi_{i \mid k, l}$.

Now let us consider the case of plane curves, i.e., $r=2$. The whole subsection should be compared with [17, Section 4], where we dealt with the special case $\Delta=d$. We fix the following notation. Let

$$
F=\left(\prod_{k=1}^{n} \tau_{a_{k}}\left(C_{k}\right)\right)_{\Delta}^{\mathbb{R}^{2}}
$$

be a one-dimensional family of plane curves with general conditions, and let $L \cup M \cup N=[n]$ be the partition of the labels such that

$$
\operatorname{codim}\left(C_{k}\right)= \begin{cases}0 & \text { if } k \in L, \\ 1 & \text { if } k \in M, \\ 2 & \text { if } k \in N\end{cases}
$$

First, we study how the deformation of a general curve $C$ in $F$ can look like.

Lemma 5.10. (Variation of [17, subsection 4.4]). Let us assume:

(i) $a_{k}=0$ for all $k \in L \cup M$, i.e., $\Psi$-conditions are only allowed together with point conditions.

Then the following holds. Let $\sigma$ be a facet of $F$, and let $C \in \sigma$ be a general curve. Then the deformation of $C$ inside $\sigma$ is described by one of the following cases: 
(I) $C$ contains a contracted bounded edge. Then the deformation inside $\sigma$ is given by changing the length of this edge arbitrarily.

(II) $C$ has a three-valent degenerated vertex $V$ of one of the following three types.

(a) One of the adjacent edges is a marked leaf, $i \in L$.

(b) One of the adjacent edges is a marked leaf, $j \in M$, and the linear spans of the corresponding line $C_{j}$ at $\mathrm{ev}_{j}(C)$ and of the other two edges adjacent to $V$ coincide (i.e., the curves $C$ and the $C_{j}$ do not intersect transversally at $\left.\mathrm{ev}_{j}(C)\right)$.

(c) All edges adjacent to $V$ are non-contracted, but their span near $V$ is still only one-dimensional; without loss of generality, we denote the edge alone on one side of $V$ by $v$ and the two edges on the other side by $v_{1}, v_{2}$.

(a)

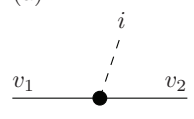

(b)

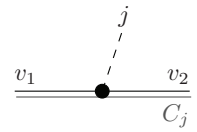

(c)

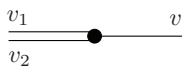

In all of these cases the deformation inside $\sigma$ is given by moving $V$.

(III) $C$ contains a movable string $S$, i.e., a two-valent subgraph of $C$ homeomorphic to $\mathbb{R}$ such that all edges are non-contracted and all vertices of $S$ are three-valent in $C$ and not degenerated in the sense of case (II). Then the deformation of $C$ is given by moving $S$ while all vertices not contained in $S$ remain fixed (in particular, only edges in or adjacent to $S$ change their lengths).

Proof. Again, for general conditions, $F$ set-theoretically coincides with the set of curves satisfying the given incidence and valence conditions. Thus, finding the deformation of $C$ inside $\sigma$ is the same as finding a way of changing the position and the length of the bounded edges of $C$ such that the resulting curve still meets the incidence conditions $C_{k}$.

It is obvious that in cases (I) and (II) changing the length of the contracted bounded edge, respectively, moving the degenerated vertex $V$ leads to such deformations.

In case (III), the non-degeneracy of the vertices makes sure that both ends of $S$ consist of non-contracted ends and that a small 
movement of one of these ends leads to a well-defined movement of the whole string (a more detailed description can be found in the proof of [17, subsection 4.4]).

Finally, this list of cases is really complete, as $C$ always contains a string whose vertices are three-valent in $C$ and whose ends are either non-contracted leaves or marked leaves in $L$. This follows from the same calculation as in [17, subsection 4.3], with the only difference that we have to replace $3 d$ by $\# \Delta$.

We have now seen how a general curve $C \in F$ can be deformed. In the second step, we will now focus on unbounded deformations.

Definition 5.11. A complete fan $\Theta$ in $\mathbb{R}^{2}$ is called del Pezzo if the associated toric surface is a smooth del Pezzo surface. Here is a complete list, up to the action of $\operatorname{SL}(2, \mathbb{Z})$.
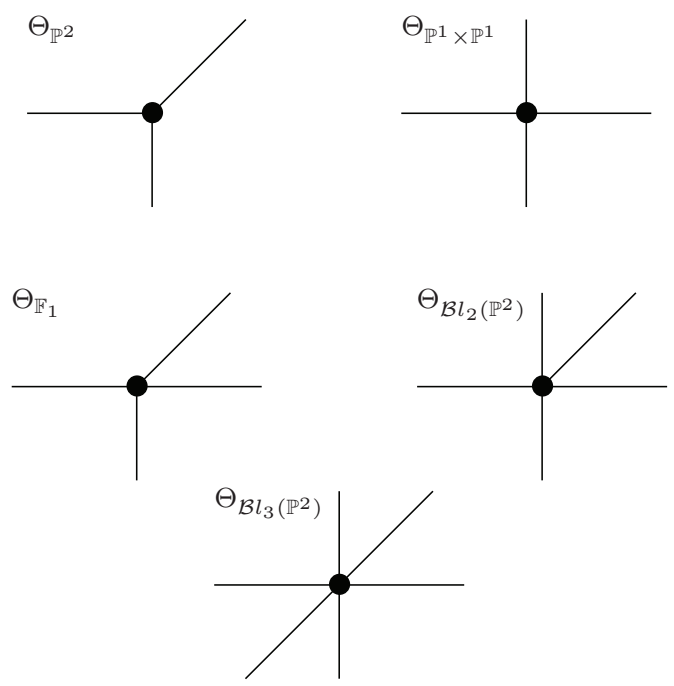

It is easy to see that an alternative characterization of these fans is as follows: Any two independent primitive vectors generating rays of $\Theta$ form a basis of $\mathbb{Z}^{2}$. A degree $\Delta$ in $\mathbb{R}^{2}$ is called del Pezzo if $\Theta(\Delta)$ is del Pezzo and if all direction vectors appearing in $\Delta$ are primitive. This ensures that, for every pair of independent vectors $v_{1}, v_{2}$ appearing in 
$\Delta$, the dual triangle to the fan spanned by $v_{1}, v_{2}$ and $-\left(v_{1}+v_{2}\right)$ does not contain lattice points apart from its vertices.

Lemma 5.12. (Variation of $[\mathbf{1 7}$, subsection 4.4]). We assume

(i) $a_{k}=0$ for all $k \in L \cup M$,

(ii) $\Delta$ is del Pezzo.

Then the following holds. Let $\sigma$ be an unbounded facet of $F$, and let $C \in \sigma$ be a general curve. Then the deformation of $C$ in $\sigma$ is described by one of the following cases:

(I) C contains a contracted bounded edge whose length can be changed arbitrarily.

(II) $C$ has a three-valent degenerated vertex $V$ of one the three types described above. Furthermore, in cases (a) and (b) (of Lemma 5.10 (II)) one of the edges $v_{1}, v_{2}$ is bounded, the other one unbounded, whereas in case (c), the edge $v$ is bounded and $v_{1}$, $v_{2}$ are unbounded.

(III) $C$ contains a moveable string $S$ with two non-contracted leaves $v_{1}, v_{2}$ and only one adjacent bounded edge $w$. The deformation of $C$ is given by increasing the length of $w$.

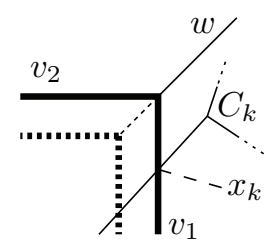

Furthermore, if $x_{k}, k \in M$, is a marked leaf adjacent to $S$, then $h\left(x_{k}\right)$ is a general point in an unbounded facet of $C_{k}$ whose outgoing direction vector $v$ lies in the interior of the cone spanned by $v_{1}, v_{2}$.

Proof. Nothing happens in cases (I), (II) (a), (b). In case (II) (c), the edge $v$ cannot be unbounded as $v=-v_{1}-v_{2}$ is not primitive. Therefore, the two edges on the other side of $V$ must be unbounded.

In case (III), the proof of the first statement is fully contained in the last part of the proof of $[\mathbf{1 7}$, subsection 4.4]. We assume that we have 
a string $S$ with two unique non-contracted ends and all of its vertices are three-valent and not degenerated in the sense of case (II). The deformation only moves the string $S$; the adjacent edges are shortened or elongated and the other parts of the curve remain fixed. We want to show that $S$ has only one adjacent bounded edge.

If there are bounded edges adjacent to $S$ to both sides of $S$ as in picture (a) below, then the movement of the string is bounded. (This is true because, if we move the string to either side, we can only move until the length of one of the adjacent bounded edges shrinks to 0.) So we only have to consider the case when all adjacent bounded edges of $S$ are on the same side of $S$, say on the right side as in picture (b) below. Label the edges of $S$ (respectively, their direction vectors) by $v_{1}, \ldots, v_{k}$ and the adjacent bounded edges of the curve by $w_{1}, \ldots, w_{k-1}$ as in the picture. As above, the movement of the string to the right is bounded. If one of the directions $w_{i+1}$ is obtained from $w_{i}$ by a left turn (as is the case for $i=1$ in the picture), then the edges $w_{i}$ and $w_{i+1}$ meet on the left of $S$. This restricts the movement of the string to the left, too, since the corresponding edge $v_{i+1}$ then shrinks to length 0 .

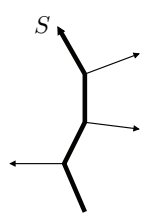

(a)

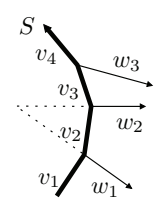

(b)

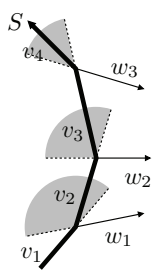

(c)

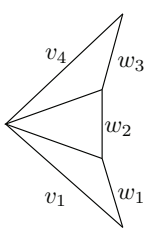

(d)

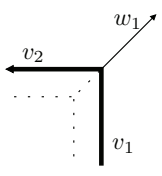

(e)

So we can assume that, for all $i$, the direction $w_{i+1}$ is either the same as $w_{i}$ or obtained from $w_{i}$ by a right turn as in picture (c). The balancing condition then shows that, for all $i$, both the directions $v_{i+1}$ and $-w_{i+1}$ lie in the angle between $v_{i}$ and $-w_{i}$ (shaded in the picture above). Therefore, all directions $v_{i}$ and $-w_{i}$ lie within the angle between $v_{1}$ and $-w_{1}$. In particular, the image of the string $S$ cannot have any self-intersections in $\mathbb{R}^{2}$. We can therefore pass to the (local) dual picture (d) where the edges dual to $w_{i}$ correspond to a concave side of the polygon whose other two edges are the ones dual to $v_{1}$ and $v_{k}$. 
But, from our assumption that $\Delta$ is del Pezzo, we know that the triangle dual to $v_{1}$ and $v_{k}$ does not contain more integer points than its vertices. We conclude that the concave side of the polygon in picture (d) actually must coincide with the triangle dual to $v_{1}$ and $v_{k}$ and, therefore, the string consists of the two ends $v_{1}$ and $v_{2}$ that are connected to the rest of the curve by exactly one bounded edge $w_{1}=w$ (as shown in picture (e)).

The second statement concerning adjacent marked leaves $x_{k}, k \in M$ is obvious as the deformation is supposed to be unbounded.

Theorem 5.13. Let $x_{i}, x_{j}, x_{k}$ and $x_{l}$ be pairwise different marked leaves, and let us assume:

(i) $a_{k}=0$ for all $k \in L \cup M$,

(ii) $\Delta$ is del Pezzo,

(iii) if $i, j \in M$ (respectively, $k, l \in M$ ), then for any pair of independent direction vectors $v_{1}, v_{2}$ appearing in $\Delta$, the interior of the cone spanned by $v_{1}$ and $v_{2}$ does not intersect both degrees $\delta\left(C_{i}\right)$ and $\delta\left(C_{j}\right)$ (respectively, $\delta\left(C_{k}\right)$ and $\delta\left(C_{l}\right)$ ).

Then $\phi_{i, j \mid k, l}$ is bounded. If we additionally require

(iv) $i \in N$,

then also $\phi_{i \mid k, l}$ is bounded.

Proof. As conditions (i) and (ii) hold, we can apply Lemma 5.12, which describes the unbounded facets of $F$. We have to show that $\phi_{i, j \mid k, l}$ (respectively, $\phi_{i \mid k, l}$ ) is bounded on these facets. In case (I), the only changing length is that of a contracted edge and therefore not measured by both $\phi_{i, j \mid k, l}$ and $\phi_{i \mid k, l}$. In case (II), the edge whose length is growing infinitely cannot separate more then one marked leaf $x_{k}, k \in L \cup M$ from the others. Therefore, this length cannot contribute to $\phi_{i, j \mid k, l}$ and, by condition iv), to $\phi_{i \mid k, l}$. Finally, condition (iii) (and also condition (iv)) is made such that $\phi_{i, j \mid k, l}$ and $\phi_{i \mid k, l}$ are also bounded in case (III). 
Remark 5.14. Conditions (i)-(iv) appearing in the above statements are not only sufficient but, in most cases, also necessary for the statements to hold.

(iv) If condition (iv) in Theorem 5.13 is not satisfied, we can get the following things.

- If $i \in L$, then the degenerated vertex of type (a) leads to an unbounded $\phi_{i \mid k, l}$.

- If $i \in M$ and $\rho$ is a ray in $C_{i}$ whose direction vector $v_{\rho}$ also appears in $\Delta$, then in general we will find curves in $F$ with a degenerated vertex of type (b), whose unbounded movement will make $\phi_{i \mid k, l}$ unbounded.

- If $i \in M$ and $\rho$ is a ray in $C_{i}$ whose direction vector $v_{\rho}$ lies between two direction vectors $v_{1}, v_{2}$ appearing in $\Delta$, this will in general lead to curves in $F$ with unbounded deformations of case (III) such that the outward directions are $v_{1}, v_{2}$ and such that $x_{i}$ is adjacent to the moved string. So, again, $\phi_{i \mid k, l}$ is in general unbounded.

(iii) If condition (iii) is not satisfied, we will in general get unbounded deformations of the following type:

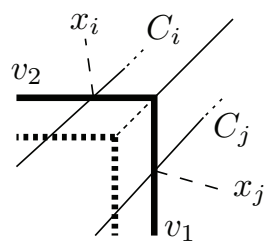

In this case, we have $i, j \in M$, and the interior of the cone spanned by $v_{1}, v_{2}$ contains direction vectors of both $C_{i}$ and $C_{j}$. As in general $x_{k}$ and $x_{l}$ will lie on the other side of the growing edge $w, \phi_{i, j \mid k, l}$ will be unbounded.

(ii) If we drop condition (ii), i.e., if we allow non-del Pezzo degrees $\Delta$, two things can happen. If we allow non-primitive direction vectors, then we get deformations of type (II) (c) with unbounded edge $v$. Therefore, the lengths of $v_{1}$ and $v_{2}$, which can in general separate arbitrary marked leaves, grow infinitely. If $\Theta(\Delta)$ is not supposed to be del Pezzo, then the description of unbounded deformations of case (III) in Lemma 5.12 becomes 
incorrect, as more complicated strings will appear with more adjacent bounded edges than just one. The example of $\mathbb{F}_{2}$ is analyzed in detail in [4] and [5, e.g., 2.10].

(i) If we drop condition (i), i.e., if we allow $\Psi$-conditions also at marked leaves which are not fixed by points, we end up with more complicated kinds of deformations of general curves in $F$. The picture below shows an example of an unbounded deformation in a one-dimensional family of plane curves of projective degree 2. Here, $C$ has to meet all four tropical lines $C_{1}, \ldots, C_{4}$ with one $\Psi$-condition. Note that the indicated deformation of $C$ is indeed unbounded and that the length of the $(1,-1)$-edge $e$ grows infinitely. This example can be extended in the following way. One can glue arbitrary (fixed) curves to the non-contracted leaves of $C$ in direction $(1,1)$, obtaining more families admitting such a deformation. In particular, the edge $e$ can separate arbitrary kinds of points, showing that, in general, $\phi_{i, j \mid k, l}$ and $\phi_{i \mid k, l}$ can be unbounded for any choice of $i, j, k, l$.

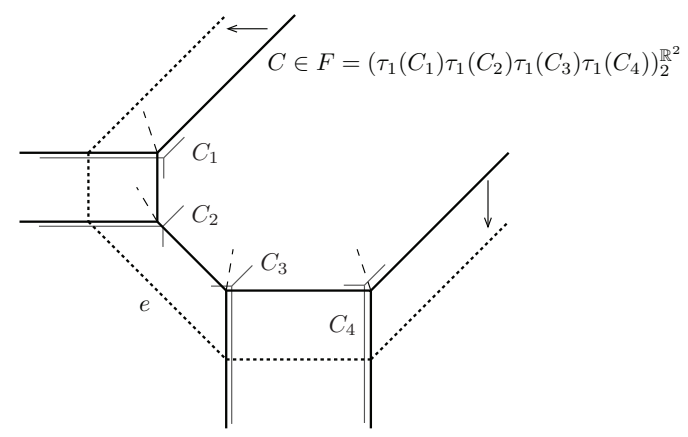

In higher dimensions $(r \geq 3)$, until now only the following case has been studied.

Theorem 5.15 ([22] 4.86). Let

$$
F=\left(\prod_{k=1}^{n} \tau_{0}\left(V_{k}\right)\right)_{d}^{\mathbb{R}^{r}}
$$


be a one-dimensional family of curves of projective degree $d$ in $\mathbb{R}^{r}$ which does not satisfy the $\Psi$-conditions, but incidence conditions given by conventional linear spaces $V_{k} \subseteq \mathbb{R}^{r}$. Then, for any choice of $\{i, j, k, l\} \in[n]$, the function $\phi_{i, j \mid k, l}$ is bounded on $F$.

5.4. Comparison to the algebro-geometric invariants. In the special case of an empty degree, denoted by $\Delta=0$, the situation is analogous to the algebro-geometric one.

Proposition 5.16. Let $Z=\left(\prod_{k=1}^{n} \tau_{a_{k}}\left(C_{k}\right)\right)_{0}$ be a zero-dimensional intersection product in $\mathcal{M}_{n}^{\mathrm{lab}}\left(\mathbb{R}^{r}, 0\right)$. Then $\operatorname{deg}(Z)$ is non-zero if and only if $\sum_{k=1}^{n} \operatorname{codim}\left(C_{k}\right)=r$ (or equivalently $\left.\sum_{k=1}^{n} a_{k}=n-3\right)$. In this case,

$$
\operatorname{deg}(Z)=\left(\begin{array}{c}
n-3 \\
a_{1}, \ldots, a_{n}
\end{array}\right) \operatorname{deg}\left(C_{1} \cdots C_{k}\right)
$$

holds.

Proof. By definition, $\mathcal{M}_{n}^{\text {lab }}\left(\mathbb{R}^{r}, 0\right)$ is isomorphic to $\mathcal{M}_{n} \times \mathbb{R}^{r}$. Moreover, as $\Delta=0$, all evaluation maps $\mathrm{ev}_{i}$ coincide with the projection onto the second factor, which we therefore denote by ev. Now, let

$$
X:=\prod_{k=1}^{n} \psi_{k}^{a_{k}}=\left(\prod_{k=1}^{n}\left(\psi_{k}^{\mathrm{abstr}}\right)^{a_{k}}\right) \times \mathbb{R}^{r}
$$

be the intersection of all $\Psi$-divisors. Then the projection formula applied to ev yields

$$
\operatorname{deg}(Z)=\operatorname{deg}\left(C_{1} \cdots C_{n} \cdot \operatorname{ev}_{*}(X)\right) .
$$

But $\mathrm{ev}_{*}(X)$ is non-zero if and only if $\sum_{k=1}^{n} a_{k}=n-3$. If so, by Remark 2.22, we know $\operatorname{ev}_{*}(X)=\left(\begin{array}{c}n-3 \\ a_{1}, \ldots, a_{n}\end{array}\right) \cdot \mathbb{R}^{r}$, which proves the statement.

Remark 5.17. The goal of the following theorem is to show that certain tropical and classical gravitational descendants coincide. The idea is to show that, under the restrictions which we accumulated in the preceding sections, both sets of numbers satisfy the same WDVV 
and topological recursion equations, which are sufficient for determining the numbers from some initial values. However, there is one further problem concerning this plan, which we already mentioned in Remark 5.5. The classical WDVV and topological recursion equations run through splittings of the given cohomology class $\beta$ into sums $\beta=\beta_{1}+\beta_{2}$. As $\bar{M}_{0, n}(\mathbf{X}, \beta)$ is empty if $\beta$ is not effective, we can restrict to effective classes $\beta, \beta_{1}, \beta_{2}$.

Now, for $\mathbb{P}^{2}$ and $\mathbb{P}^{1} \times \mathbb{P}^{1}$, affectivity is equivalent to the fact that the associated one-dimensional tropical fans are positive (as $\mathbb{P}^{2}$ and $\mathbb{P}^{1} \times \mathbb{P}^{1}$ do not contain curves with negative self-intersection). So a splitting $\beta=\beta_{1}+\beta_{2}$ of effective cohomology classes corresponds bijectively to a sum of unlabeled tropical degrees $\delta=\delta_{1}+\delta_{2}$, and therefore the tropical and classical equations are really equivalent in this case.

However, for the blow ups of $\mathbb{P}^{2}$ in up to three torus-fixed points (i.e., for $\mathbb{F}_{2}, \mathcal{B} l_{2}\left(\mathbb{P}^{2}\right)$ and $\mathcal{B} l_{3}\left(\mathbb{P}^{2}\right)$, cf., Definition 5.11), the same argument fails as the exceptional divisors induce tropical fans with negative weights. The following picture shows an example of the tropical fan associated to the exceptional divisor $V(\varrho)$ of $\mathbb{F}_{1}$.
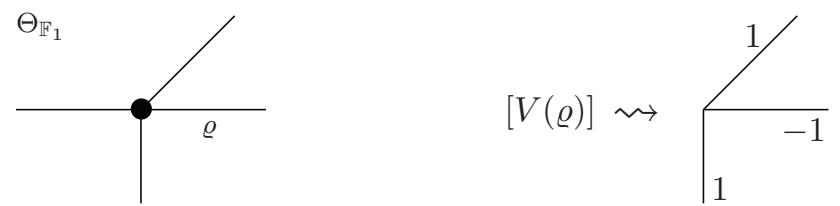

In these cases, i.e., when classical curves can split into reducible curves with a rigid component in the toric boundary, we cannot expect that our purely non-compact approach will yield the same results. It should be possible to deal with this by (partially) compactifying our spaces and/or adding suitable correction terms (as in [5]). This needs to be addressed in further work. For now, we just restrict ourselves to $\mathbb{P}^{2}$ and $\mathbb{P}^{1} \times \mathbb{P}^{1}$.

Now we are finally ready to compare the tropical invariants for plane tropical curves to the algebro-geometric ones, for some cases, using the equations proven in the previous subsections. The theorem 
is an extension of [17, Theorem 8.4], which proves the statement for the case $\mathbb{P}^{2}$.

Theorem 5.18. Let

- $\Theta$ be $\Theta_{\mathbb{P}^{2}}$ or $\Theta_{\mathbb{P}^{1} \times \mathbb{P}^{1}}$, and set $\mathbf{X}:=\mathbf{X}(\Theta)$ (i.e., $\mathbf{X}=\mathbb{P}^{2}$ or $\left.\mathbf{X}=\mathbb{P}^{1} \times \mathbb{P}^{1}\right)$

- $C_{1}, \ldots, C_{n}$ be $\Theta$-directional tropical cycles, and let $\gamma_{1}, \ldots, \gamma_{n} \in$ $A^{*}(\mathbf{X})$ be the associated cohomology classes of $\mathbf{X}$,

- $\Delta$ be a labeled degree with primitive direction vectors whose unlabeled degree $\delta(\Delta)$ is $\Theta$-directional, and let $\beta \in A^{r-1}(\mathbf{X})$ be the corresponding cohomology class,

- $a_{1}, \ldots, a_{n}$ be non-negative integers such that $a_{k}=0$ if $\operatorname{dim}\left(C_{k}\right)$ $>0$.

Then the tropical and algebro-geometric gravitational descendants are equal, i.e.,

$$
\frac{1}{\Delta !}\left\langle\tau_{a_{1}}\left(C_{1}\right) \cdots \tau_{a_{n}}\left(C_{n}\right)\right\rangle_{\Delta}^{\mathbb{R}^{2}}=\left\langle\tau_{a_{1}}\left(\gamma_{1}\right) \cdots \tau_{a_{n}}\left(\gamma_{n}\right)\right\rangle_{\beta}^{\mathbf{X}}
$$

Proof. First we choose a basis $B_{0}, \ldots, B_{m}$ of $Z_{*}(\Theta)$. This also determines a basis $\eta_{0}, \ldots, \eta_{m}$ of $A^{*}(\mathbf{X})$, and we know from comparison to the fan displacement rule (cf., Theorem 1.9) that

$$
\operatorname{deg}\left(B_{e} \cdot B_{f}\right)=\operatorname{deg}\left(\eta_{e} \cdot \eta_{f}\right)
$$

holds. This implies that, if we use WDVV equations or topological recursion with respect to these bases, then the diagonal coefficients $\beta_{\text {ef }}$ appearing in the tropical and in the algebro-geometric setting coincide. Thus, using the results of the previous sections, we know that the numbers $1 / \Delta !\left\langle\tau_{a_{1}}\left(C_{1}\right) \cdots \tau_{a_{n}}\left(C_{n}\right)\right\rangle_{\Delta}=\left\langle\tau_{a_{1}}\left(C_{1}\right) \cdots \tau_{a_{n}}\left(C_{n}\right)\right\rangle_{\delta(\Delta)}$ and $\left\langle\tau_{a_{1}}\left(\gamma_{1}\right) \cdots \tau_{a_{n}}\left(\gamma_{n}\right)\right\rangle_{\beta}^{\mathbf{X}}$ satisfy a certain set of identical equations, namely, the WDVV and topological recursion equations (where on the tropical side we have to be slightly more careful about $i, j, k, l$ satisfying conditions (iii) and (iv) of Theorem 5.13) as well as the string and divisor equation. Therefore, we can finish the proof by showing that the numbers can be computed recursively, using these equations, from some initial numbers and proving that these initial numbers coincide. 
We separate the labels of the marked leaves into the sets $L \cup M \cup N$ $=[n]$ according to the dimension of $C_{k}$ as in subsection 5.3. First, we use topological recursion to reduce the number of $\Psi$-conditions. We pick a marked leaf $x_{i}$ with $a_{i}>0$ (and therefore $i \in N$ ) and an arbitrary pair of marked leaves $x_{k}, x_{l}$ satisfying condition (iii) of Theorem 5.13. If such $x_{k}, x_{l}$ do not exist, we can add them using the divisor equation backwards with appropriate rational functions $h_{k}, h_{l}$. Namely, if $\mathbf{X}=\mathbb{P}^{1} \times \mathbb{P}^{1}$, we can use $h_{k}=h_{l}=\max \{0, x, y, x+y\}$; otherwise, we can use $h_{k}=h_{l}=\max \{0, x, y\}$. Note also that this choice ensures that $h_{k} \cdot \Delta=h_{l} \cdot \Delta$ is non-zero for every possible degree, so we do not divide by zero.

After eliminating all $\Psi$-conditions in this way, we can assume $a_{k}=0$ for all $k \in[n]$, i.e., we are back in the case of usual (primary) GromovWitten invariants. After applying the string and divisor equation we can assume that $L=M=\emptyset$, and it remains to compute invariants of the form $\left\langle\prod_{k=1}^{n} \tau_{0}\left(P_{k}\right)\right\rangle_{\Delta}$ for points $P_{1}, \ldots, P_{n} \in \mathbb{R}^{2}$. Comparison of dimensions shows $\# \Delta=n+1$.

Let us first consider the general case $n \geq 3$. Here we consider the one-dimensional family $F=\left(\tau_{0}\left(C_{i}\right) \tau_{0}\left(C_{j}\right) \prod_{k=1}^{n-1} \tau_{0}\left(P_{k}\right)\right)_{\Delta}$ with arbitrary $\Theta$-directional curves $C_{i}$ and $C_{j}$ such that $C_{i} \cdot C_{j}$ is nonzero and such that condition (iii) of Theorem 5.13 is satisfied (e.g., we can choose the divisors of the functions chosen above). We let $x_{i}, x_{j}$ be the first two marked leaves as indicated, and choose $k, l \in[n-1]$ arbitrarily. In the corresponding WDVV equation only one extremal partition $I \mid J$ with $\Delta_{I}=0$ and $\Delta_{J}=\Delta$ does not vanish. This follows from Lemma 5.16 and the fact that the three sums $\operatorname{codim}\left(P_{k}\right)+\operatorname{codim}\left(P_{l}\right), \operatorname{codim}\left(C_{i}\right)+\operatorname{codim}\left(P_{k}\right), \operatorname{codim}\left(C_{j}\right)+\operatorname{codim}\left(P_{l}\right)$ are greater than 2. Moreover, the only remaining extremal partition $I=\{i, j\}, J=\Delta \cup[n-1]$ provides the term

$$
\begin{aligned}
\left\langle\tau_{0}\left(C_{i}\right) \tau_{0}\left(C_{j}\right) \tau_{0}\left(\mathbb{R}^{2}\right)\right\rangle_{0} \cdot\left\langle\tau_{0}(P)\right. & \left.\prod_{k=1}^{n-1} \tau_{0}\left(P_{k}\right)\right\rangle_{\Delta} \\
& =\operatorname{deg}\left(C_{i} \cdot C_{j}\right) \cdot\left\langle\prod_{k=1}^{n} \tau_{0}\left(P_{k}\right)\right\rangle_{\Delta} .
\end{aligned}
$$


Hence, we can reduce the computation of $\left\langle\prod_{k=1}^{n} \tau_{0}\left(P_{k}\right)\right\rangle_{\Delta}$ to invariants of smaller degree. We can repeat this until we arrive at the initial invariants with $n=1$ or $n=2$. In these cases, $\# \Delta=2$ or $\# \Delta=3$, and therefore the only possible degrees (up to identification via linear isomorphisms of $\mathbb{Z}^{2}$ ) are $\Delta=\left\{-e_{1}, e_{1}\right\}$ and $\Delta=\left\{-e_{1},-e_{2}, e_{1}+e_{2}\right\}$. In both cases, it is easy to show by direct computation that $\left\langle\tau_{0}\left(P_{1}\right)\right\rangle_{\Delta}=1$ and $\left\langle\tau_{0}\left(P_{1}\right) \tau_{0}\left(P_{2}\right)\right\rangle_{\Delta}=1$ hold (given a point in $\mathbb{R}^{2}$, there is exactly one horizontal line through it; given two points, there is exactly one tropical line connecting them). But now, as discussed above, the same recursion for the classical numbers proves the claim.

Remark 5.19 (Multiplicities of tropical curves). The above theorem reduces the computation of the classical gravitational descendants to the count of certain tropical curves $C$ with multiplicities mult $(C)$ (cf., Remark 3.10). In the above case of plane curves, an easy formula for this multiplicity exists (cf., [17, Lemma 9.3]). Namely, if we assume the general position, the multiplicity of a curve in the count is obtained as the product

$$
\operatorname{mult}(C)=\prod_{V} \operatorname{mult}(V)
$$

where the product runs through all vertices to which no marked leaf is adjacent and mult $(V)$ of these necessarily 3 -valent vertices is the well-known vertex multiplicity introduced by Mikhalkin (cf., [18, Definition 2.16]). This is correct for labeled curves $C$, but we can as well count unlabeled curves $\widetilde{C}$ (as the incidence and valence conditions do not depend on the labeling) by setting

$$
\operatorname{mult}(\widetilde{C})=\frac{1}{\# \operatorname{Aut}(\widetilde{C})} \operatorname{mult}(C)
$$

Here \# Aut $(\widetilde{C})$ denotes the number of automorphisms of $\widetilde{C}$.

Moreover, as well as for the usual Gromov-Witten invariants considered in [18], there exists a so-called lattice path algorithm to compute these counts easily (cf., [17, section 9]).

Remark 5.20. Similarly we can deal with the case $r=1$, i.e., we can prove 


$$
\frac{1}{d !^{2}}\left\langle\tau_{0}\left(\mathbb{R}^{1}\right)^{l} \prod_{k=1}^{n} \tau_{a_{k}}\left(P_{k}\right)\right\rangle_{d}^{\mathbb{R}^{1}}=\left\langle\tau_{0}\left(\left[\mathbb{P}^{1}\right]\right)^{l} \prod_{k=1}^{n} \tau_{a_{k}}([p t])\right\rangle_{d}^{\mathbb{P}^{1}}
$$

where the left hand side is a tropical, the right hand side a conventional invariant and $[p t]$ denotes the class of a point $p t \in \mathbb{P}^{1}$. In fact, after applying the string equation, we are left with the case where $l=0$. Now we use Proposition 5.9 and topological recursion to reduce the number of $\Psi$-conditions (where, if $n<3$, we first add more marked leaves using the divisor equation). Finally, when $a_{k}=0$ for all $k \in[n]$, it follows that $d=1$, and we can directly compute $\left\langle\tau_{0}(P)\right\rangle_{1}^{\mathbb{R}^{1}}=1$.

This fits with the previously known result for rational Hurwitz numbers $H_{d}^{0}:=\left\langle\tau_{1}([p t])^{2 d-2}\right\rangle_{d}^{\mathbb{P}^{1}}$ (cf., [3, Lemma 9.7]).

Remark 5.21. The discussion in Remark 5.14 and the factor $n+$ $\# \Delta-2$ appearing in the tropical dilaton equation (3.12), instead of $n-2$ in the algebro-geometric version, show that for more difficult degrees $\Delta$ (if $r=2$ ) and for $\Psi$-conditions at marked leaves $x_{k}$ with $\operatorname{dim}\left(C_{k}\right)>0$, the corresponding tropical and conventional invariants are in general different. For example, if we add a marked leaf that has to satisfy only a $\Psi$-condition, the different factors in the dilaton equations immediately lead to different invariants.

Remark 5.22. Of course, the machinery developed here is ready to use in higher dimensions as well. For example, by Remark 4.19 and Theorem 5.15, the same approach can be used to show that tropical and classical Gromov-Witten invariants (without $\Psi$-classes) of $\mathbb{P}^{r}, r$ arbitrary, coincide.

Acknowledgments. I would like to thank Andreas Gathmann, Eric Katz, Michael Kerber, Hannah Markwig and Grigory Mikhalkin for many helpful discussions and/or for proofreading various versions of this work.

\section{REFERENCES}

1. Lars Allermann and Johannes Rau, First steps in tropical intersection theory, Math. Z. 264 (2010), 633-670. 
2. Lars Allermann and Johannes Rau, Tropical rational equivalence on $\mathbb{R}^{r}$, preprint, arxiv:0811.2860.

3. Renzo Cavalieri, Paul Johnson and Hannah Markwig, Tropical Hurwitz numbers, J. Alg. Comb. 32 (2010), 241-265, also at arxiv:0804.0579.

4. Marina Franz, The tropical Kontsevich formula for toric surfaces, Ph.D. thesis, TU Kaiserslautern, 2008.

5. Marina Franz and Hannah Markwig, Tropical enumerative invariants of $\mathbb{F}_{0}$ and $\mathbb{F}_{2}$, Adv. Geom. 11 (2011), 49-72.

6. William Fulton and Rahul Pandharipande, Notes on stable maps and quantum cohomology, Proc. Symp. Pure Math. 62 (1997), 45-96.

7. William Fulton and Bernd Sturmfels, Intersection theory on toric varieties, Topology 36 (1997), 335-353.

8. Andreas Gathmann, Michael Kerber and Hannah Markwig, Tropical fans and the moduli spaces of tropical curves, Comp. Math. 145 (2009), 173-195.

9. Andreas Gathmann and Hannah Markwig, Kontsevich's formula and the WDVV equations in tropical geometry, Adv. Math. 217 (2008), 537-560.

10. Andreas Gathmann and Eva-Maria Zimmermann, The WDVV equations in tropical geometry, in preparation.

11. Matthias Herold, Intersection theory of the tropical moduli spaces of curves, Ph.D. thesis, TU Kaiserslautern, 2007.

12. Eric Katz, A tropical toolkit, Expo. Math. 27 (2009), 1-36.

13. - Tropical intersection theory from toric varieties, preprint, arxiv: 0907.2488

14. Michael Kerber and Hannah Markwig, Counting tropical elliptic plane curves with fixed $j$-invariant, Comm. Math. Helv. 84 (2009), 387-427.

15. Intersecting Psi-classes on tropical $M_{0, n}$, Int. Math. Res. Not. 2009, 221-240.

16. Joachim Kock and Israel Vainsencher, An Invitation to quantum cohomology, Progr. Math. 249, 2007.

17. Hannah Markwig and Johannes Rau, Tropical descendant Gromov-Witten invariants, Manuscr. Math. 129 (2009), 293-335.

18. Grigory Mikhalkin, Enumerative tropical geometry in $\mathbb{R}^{2}$, J. Amer. Math. Soc. 18 (2005), 313-377.

19. $827-852$.

20. Moduli spaces of rational tropical curves, preprint, arxiv:0704.0839.

21. David Speyer and Bernd Sturmfels, Tropical Grassmannians, Adv. Geom. 4 (2004), 389-411.

22. Eva-Maria Zimmermann, Generalizations of the tropical Kontsevich formula to higher dimensions, Ph.D. thesis, TU Kaiserslautern, 2007. 
Johannes Rau, Universität des SaArlandes, Fachrichtung Mathematik, Postfach 151150, 66041 Saarbrǘcken, Germany

Email address: johannes.rau@math.uni-sb.de 